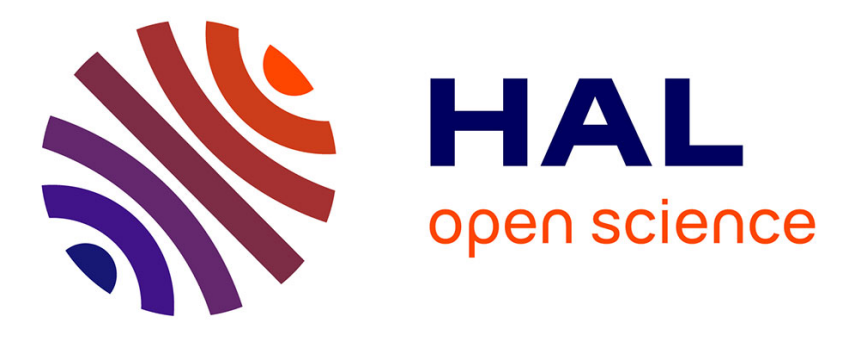

\title{
Zero-Knowledge Elementary Databases with More Expressive Queries
}

Benoît Libert, Khoa Nguyen, Benjamin Hong Meng Tan, Huaxiong Wang

\section{To cite this version:}

Benoît Libert, Khoa Nguyen, Benjamin Hong Meng Tan, Huaxiong Wang. Zero-Knowledge Elementary Databases with More Expressive Queries. PKC 2019 - 22nd IACR International Conference on Practice and Theory of Public-Key Cryptography, Apr 2019, Beijing, China. pp.255-285, 10.1007/978-3-030-17253-4_9. hal-02151645

\section{HAL Id: hal-02151645 \\ https://hal.inria.fr/hal-02151645}

Submitted on 9 Jun 2019

HAL is a multi-disciplinary open access archive for the deposit and dissemination of scientific research documents, whether they are published or not. The documents may come from teaching and research institutions in France or abroad, or from public or private research centers.
L'archive ouverte pluridisciplinaire HAL, est destinée au dépôt et à la diffusion de documents scientifiques de niveau recherche, publiés ou non, émanant des établissements d'enseignement et de recherche français ou étrangers, des laboratoires publics ou privés. 


\title{
Zero-Knowledge Elementary Databases with More Expressive Queries
}

\author{
Benoit Libert ${ }^{1,2}$, Khoa Nguyen ${ }^{3}$, Benjamin Hong Meng Tan ${ }^{3,4}$, and \\ Huaxiong Wang ${ }^{3}$ \\ 1 CNRS, Laboratoire LIP, France \\ 2 ENS de Lyon, Laboratoire LIP (U. Lyon, CNRS, ENSL, INRIA, UCBL), France \\ 3 School of Physical and Mathematical Sciences, Nanyang Technological University, \\ Singapore \\ 4 Institute for Infocomm Research, A*STAR, Singapore
}

\begin{abstract}
Zero-knowledge elementary databases (ZK-EDBs) are cryptographic schemes that allow a prover to commit to a set D of key-value pairs so as to be able to prove statements such as " $x$ belongs to the support of $\mathrm{D}$ and $\mathrm{D}(x)=y$ " or " $x$ is not in the support of $\mathrm{D}$ ". Importantly, proofs should leak no information beyond the proven statement and even the size of D should remain private. Chase et al. (Eurocrypt'05) showed that ZK-EDBs are implied by a special flavor of non-interactive commitment, called mercurial commitment, which enables efficient instantiations based on standard number theoretic assumptions. On the other hand, the resulting ZK-EDBs are only known to support proofs for simple statements like (non-)membership and value assignments. In this paper, we show that mercurial commitments actually enable significantly richer queries. We show that, modulo an additional security property met by all known efficient constructions, they actually enable range queries over keys and values - even for ranges of super-polynomial size as well as membership/non-membership queries over the space of values. Beyond that, we exploit the range queries to realize richer queries such as $k$-nearest neighbors and revealing the $k$ smallest or largest records within a given range. In addition, we provide a new realization of trapdoor mercurial commitment from standard lattice asssumptions, thus obtaining the most expressive quantum-safe ZK-EDB construction so far.
\end{abstract}

Keywords. Zero-knowledge databases, expressive queries, lattice-based commitments.

\section{Introduction}

Zero-knowledge sets (ZKS), as introduced by Micali, Rabin and Kilian 21, allow a prover $P$ to commit to a finite set $S$ without revealing its size. The commitment is generated such that the prover can efficiently and non-interactively prove the membership or non-membership of certain elements $x$ in the committed set $S$. The zero-knowledge property mandates that proofs reveal no information beyond the truth of the statement: even its cardinality should remain hidden. The 
soundness property captures the prover's inability to prove contradictory statements " $x \in S$ " and " $x \notin S$ " about the same $S$.

Zero-knowledge elementary databases (ZK-EDBs) generalize the notion of zero-knowledge sets to elementary databases (EDBs). An EDB D is a partial function: a set of key-value pairs $(x, y)$ where each key $x$ of the universe occurs at most once and thus takes at most one value $y=\mathrm{D}(x)$. For syntactic reasons, keys $x$ not in $\mathrm{D}$ are assigned $\mathrm{D}(x)=\perp$. Each query $x$ obtains a response $\mathrm{D}(x)$ and a proof of its correctness. Again, proofs should reveal no information beyond the value $\mathrm{D}(x)$ : particularly the number of records in $\mathrm{D}$. Here, soundness requires the infeasibility of proving two distinct values $y, y^{\prime}$ for any given $x$. Micali et al. 21] described an elegant construction of ZK-EDB based on the discrete logarithm assumption, which was generalized by Chase et al. [5]6 to a general design of ZK-EDBs from a lower-level primitive called mercurial commitment.

In short, mercurial commitments are commitment schemes which generate commitments in either a hard or soft mode. The former satisfies the usual binding property while the latter allows the sender to create dummy commitments that do not commit the sender to any message. The ZK-EDB constructions of 211516] combine mercurial commitments with a Merkle tree [20, where each internal node contains a mercurial commitment to its two children. The existence of dummy commitments is exactly what allows the sender to commit to the database in polynomial time without revealing its size. The latter is hidden by having a super-polynomial upper bound on the number of leaves in the Merkle tree. Each leaf is assigned to a key $x$ and contains a real commitment to the value $y=\mathrm{D}(x)$ and every internal node contains a commitment to its two children. By storing a dummy commitment at the root of each empty subtree, the sender is able to commit to the entire $\mathrm{D}=\{(x, y)\}$ in polynomial time.

While efficient and based on standard assumptions, the ZK-EDB realizations of [2115]6] have relatively limited expressivity; only simple statements like " $x$ does not belong in $\mathrm{D}$ " or " $x$ is in $\mathrm{D}$ with value $y=\mathrm{D}(x)$ " can be proved. In this paper, we show that mercurial commitments actually enable proofs of more involved statements like range queries over keys and values as well as $k$-nearest neighbour and $k$-minimum/maximum queries. As special cases, our techniques make it possible to prove membership or non-membership over values, which was not known to be possible without revealing the database size.

OuR CONTRIBUtion. In this paper, we investigate the extent to which expressive queries can be proven with efficient ZK-EDB protocols from mercurial commitments. We extend the constructions of 21156] to allow the prover to convincingly answer queries of the form "Give me all database records $(x, y) \in \mathrm{D}$ whose keys $x$ lie within the range $\left[a_{x}, b_{x}\right]$ ". For any $\left[a_{x}, b_{x}\right]$ of super-polynomial length, we show that a simple tweak in mercurial commitments allows efficient, polynomialsized proofs of correctness of the response without leaking the database size.

In a second step, we extend this technique so as to handle range queries over values. Namely, for a super-polynomially large interval $\left[a_{y}, b_{y}\right]$, we allow the prover to answer queries "Send me all records $(x, y) \in \mathrm{D}$ with values $y$ in the interval $\left[a_{y}, b_{y}\right]$ ". Again, we can prove correctness of the response in zero- 
knowledge with a polynomial-size proof. As a special case of range queries over values, we can prove statements like "No key $x$ of the database is assigned the value $y$ " or " $y$ occurs in $\mathrm{D}$ and the corresponding set of keys is $\mathrm{D}^{-1}(y)$ ". We note that previous ZK-EDB protocols 21/5/6] were unable to handle such statements while hiding the database size: the only way to prove that no record of the form $(*, y)$ exists was to prove inequalities $y_{i} \neq y$ for all records $\left(x_{i}, y_{i}\right)$.

In a third step, we also handle range queries over records. Namely, each query consists of a "narrow" rectangle $\left[a_{x}, b_{x}\right] \times\left[a_{y}, b_{y}\right]$ and the response consists of all records $(x, y)$ such that $x \in\left[a_{x}, b_{x}\right]$ and $y \in\left[a_{y}, b_{y}\right]$. Here, we can handle rectangles of polynomial width $\left[a_{y}, b_{y}\right]$ and super-polynomial height $\left[a_{x}, b_{x}\right]$ with a proof size which is linear in the size of $\left[a_{y}, b_{y}\right]$ and the number of records in $\left[a_{x}, b_{x}\right] \times\left[a_{y}, b_{y}\right]$. However, the proof length does not depend on $\left(b_{x}-a_{x}\right)$, allowing it to be very large. As a special case $[x, x] \times[y, y]$ of range query over records, we can efficiently prove that specific records $(x, y)$ do not belong to $\mathrm{D}$, which amounts to saying "if $x$ is in $\mathrm{D}$ at all, the corresponding value is not $y$ ". On top of that, we show that the proofs from the range queries described above enable several more interesting queries such as $k$-nearest neighbour and $k$-minimum/maximum. The first seeks the $k$ nearest keys, values or records from a given key, value or record while the latter asks for the $k$ smallest or largest keys, values or records within an input range. The proofs of correctness for these queries comprise of a set of range proofs that guarantees that there are no other keys, values or records between the upper (resp. lower) bound of the range and the smallest (resp. largest) record returned. In the following, we refer to ZK-EDB protocols supporting such richer queries as "Zero-knowledge expressive elementary database" (ZK-EEDB).

We insist on building ZK-EEDBs without interaction or random oracles: as in 21|5]6, only a common reference string is assumed, which is necessary for NIZK proofs in the standard model anyway [1]. Our constructions are instantiable with existing mercurial commitments based on standard number theoretic assumptions. We identify a new equivocation property of mercurial commitments which is actually present in a generic construction of trapdoor mercurial commitment from $\Sigma$-protocols due to Catalano et al. 22. Since the number theoretic constructions of [215|6] can be seen as instantiations of the general construction of [2, this immediately provides us with ZK-EEDBs based on the discrete logarithm and factoring/RSA assumptions. In addition, we provide a new construction of trapdoor mercurial commitment (TMC) based on a well-studied assumption in standard (i.e., non-ideal) lattices. Our new lattice-based TMC is a direct construction, which is not implied by the generic construction of [2]; rather, it draws inspiration from [21]. In non-ideal lattices, it performs better than TMC schemes implied by 2 under the same assumptions.

Our Techniques. Our setting involves a database owner who publishes a short string $\operatorname{com}_{\mathrm{D}}$ that commits him to a particular database D consisting of records, which are key-value pairs $(x, \mathrm{D}(x))$, where $x, \mathrm{D}(x) \in\left[0,2^{\ell}\right)$. The prover is required to answer queries and prove that the response is consistent with the committed database $\mathrm{D}$ in zero-knowledge, including not revealing how many 
keys $x$ are in the support [D] of D. For this purpose, we follow the approach of using mercurial commitments 56 .

In mercurial commitments, the binding property is relaxed by allowing the committer to softly open a commitment and say "The commitment opens to this message if it can be opened at all". During the commitment phase, the sender can either create a hard commitment, which can be hard/soft-opened to a unique message, or a soft commitment, which it can soft-open to any message. Unlike soft commitments, hard commitments can be opened both in the soft and the hard way, but soft openings can never contradict hard ones. Besides, hard and soft commitments should be computationally indistinguishable.

When a Merkle tree has a super-polynomial number of leaves, the prover has to store a soft commitment at the root of each empty sub-tree in order to commit to an EDB in polynomial time. In order to prove that some key is not in the database, the prover can soft-open all soft commitments on the path that connects the corresponding leaf to the root while generating the missing soft commitments at the time of proving non-membership.

When it comes to generating a proof for a range query $\left[a_{x}, b_{x}\right]$ over keys, the difficulty is to find a way to convince a verifier that no key of $\left[a_{x}, b_{x}\right]$ was omitted in the response. If $\left[a_{x}, b_{x}\right]$ is super-polynomially large, we cannot generate proofs of non-membership for all elements of $\left[a_{x}, b_{x}\right]$ that are not in the support [D] of D. Our solution to this problem is to rely on the Subset Cover framework of Naor, Naor and Lotspiech 25] and find the smallest set of nodes $\mathcal{P}$ that contains an ancestor of all leaves $\left[a_{x}, b_{x}\right] \backslash[\mathrm{D}]$ and no ancestor of those in $\left[a_{x}, b_{x}\right] \cap[\mathrm{D}]$. For each node $x \in \mathcal{P}$, we can have the prover convince the verifier that the soft commitment associated to $x$ (which is created if it did not exist yet and authenticated via a path from $x$ to the root) is really a soft commitment, by revealing the soft-commitment coins. For the sake of proving the zero-knowledge property, we need that the simulator be able to create fake commitments which can be subsequently equivocated by revealing fake hard/soft openings or pretending that they were soft commitments. For this purpose, we thus define a new equivocation property of mercurial commitments by requiring that fake commitments be not only equivocable as defined by prior works [2], but also "explainable" as soft commitments by using a trapdoor to compute plausible soft commitment coins. Fortunately, all known trapdoor mercurial commitments based on standard assumptions [215] satisfy this additional equivocation property. By using the Complete Subtree technique of Naor et al. 25], we are able to prove range queries $\left[a_{x}, b_{x}\right]$ in zero-knowledge with proofs of size $O\left(\ell \cdot|\mathfrak{R}| \cdot \log \left(b_{x}-a_{x}\right)\right)$, where $\mathfrak{R}=\left[a_{x}, b_{x}\right] \cap[\mathrm{D}]$ and $\ell$ is the height of the Merkle tree.

In order to handle range queries over values, our idea is to have the prover commit to $\mathrm{D}$ by generating two Merkle trees. While the first one is computed in the same way as in ordinary ZK-EDBs, the second tree is used as a "reversed database" $D^{-1}$ : namely, the keys of $D^{-1}$ are the values $y$ of $D$ and their values are ZKS commitments to all the keys $x \in \mathrm{D}^{-1}(y)$ such that $(x, y) \in \mathrm{D}$. The reversed database $\mathrm{D}^{-1}$ thus uses nested Merkle trees in that each leaf $y$ of $\mathrm{D}^{-1}$ may be assigned a value $\operatorname{com}_{\mathrm{D}_{y}^{-1}}$, which is itself a size-hiding Merkle tree commitment 
whose non-empty leaves contain the keys $x$ of $\mathrm{D}$ that map to $y$. Of course, we need to prevent the prover from cheating by using inconsistent Merkle trees in the two commitments $\operatorname{com}_{\mathrm{D}}$ and $\operatorname{com}_{\mathrm{D}^{-1}}$. To this end, we thus have proofs of membership consist of authentication paths in the two Merkle trees. By doing so, we can show that no dishonest prover can prove contradictory statements without breaking the binding property of the mercurial commitment scheme. Our NIZK proofs for range queries readily carry over to prove the correctness of responses to range queries over values $\left[a_{y}, b_{y}\right]$. In particular, it yields a simple method of proving that a given value is not reached by the partial function $D$.

Our lattice-based trapdoor mercurial commitment is statistically hiding and computationally binding under the Short-Integer-Solution (SIS) assumption [24. It builds on the lattice-based trapdoor commitment (KTX) of Kawachi et al. [16] and Micciancio-Peikert trapdoors [22]. While partially inspired by the discretelog-based construction of [5, it is a direct construction with large message space which is not implied by the generic constructions of [5]6[2].

Intuitively, we generate two public matrices $\mathbf{A}_{0}, \mathbf{A}_{1}$, the former to be applied to messages and the latter to determine the mode of the commitment. When producing a commitment to some message, using a random matrix $\mathbf{R}$, we first compute a matrix $\mathbf{B}=\left[\mathbf{A}_{1} \mid \mathbf{B}_{1}\right]$, where $\mathbf{B}_{1}=\mathbf{A}_{1} \mathbf{R}\left(\operatorname{resp} . \mathbf{B}_{1}=\mathbf{G}-\mathbf{A}_{1} \mathbf{R}\right)$ if the commitment is a hard (resp. soft) one. The pair $\mathbf{A}_{0}, \mathbf{B}$ can be considered the public key of an instance of the KTX commitment scheme, with an associated trapdoor for $\mathbf{B}$ if the mercurial commitment is a soft one.

A mercurial commitment to a message, $\boldsymbol{\mu}$, is a commitment, "public key" pair, $\mathbf{C}=\left(\mathbf{c}=\mathbf{A} \boldsymbol{\mu}+\mathbf{B r}, \mathbf{B}_{1}\right)$ for some commitment randomness $\mathbf{r}$. The two flavors of openings are straightforward: Soft openings to $\boldsymbol{\mu}$ are simply openings of $\mathbf{c}$ to $\boldsymbol{\mu}$ with the associated "public key" $\mathbf{A}_{0}, \mathbf{B}=\left[\mathbf{A} \mid \mathbf{B}_{1}\right]$. Hard openings, on the other hand, have an additional step of showing that $\mathbf{B}_{1}=\mathbf{A}_{1} \mathbf{R}$ for some $\mathbf{R}$, essentially demonstrating that the "public key" does not have an embedded trapdoor.

Catalano et al. [2, Section 5] built a TMC scheme with large message space from any trapdoor commitment where a $\Sigma$-protocol allows proving knowledge of an opening to 0 . For this purpose, the $\Sigma$-protocol is required to have a large challenge space, which becomes the message space of the TMC scheme. In the lattice setting, the only known $\Sigma$-protocols [19] with large challenge space operate over ideal lattices and thus require less standard assumptions than non-ideal lattices. Moreover, their honest-verifier zero-knowledge property relies on the prover performing rejection sampling and outputting a simulated transcript only with some probability, say $1 / c$, for some constant $c$. Since the TMC scheme of [2, Section $5]$ generates hard commitments by running the HVZK simulator of the underlying $\Sigma$-protocol, the hard-committer can only produce a properly distributed hard commitment after $c$ attempts on average. Our TMC scheme eliminates the need for several attempts and only requires one attempt to generate a hard commitment.

Related Work. Ostrovsky, Rackoff and Smith [28] described protocols handling orthogonal multi-dimensional range queries for committed databases allow- 
ing for $d$-dimensional key spaces. While their protocols extend to provide privacy by means of zero-knowledge proofs, they do not hide the database size. Chase $e t$ al. [56] and Catalano et al. [2] described size-hiding constructions of ZK-EDBs under general assumptions. In particular, Catalano, Dodis and Visconti 2 gave simplified security definitions for (trapdoor) mercurial commitments and showed how to obtain them from one-way functions in the shared random string model. An EDB D is a partial function: a set of key-value pairs $(x, y)$ where each key $x$ of the universe occurs at most once and thus takes at most one value $y=\mathrm{D}(x)$.

Liskov [18] considered the notion of updatable zero-knowledge databases in the random oracle model. Prabhakaran and Xue [31] put forth the similar notion of statistically hiding sets, which allows for more efficient constructions. For the sake of efficiency, Kate et al. [15] considered quasi-database commitments which do not aim at hiding the database size. Catalano, Fiore and Messina [4] suggested a technique for compressing proofs of non-membership in ZK-EDB protocols. Libert and Yung [17] extended their idea to compress both proofs of membership and non-membership, while Catalano and Fiore [3] achieved similar proof compressions under more standard number theoretic assumptions.

An orthogonal line of work investigated the feasibility of stronger definitions in size-hiding database commitments. Gennaro and Micali [9 formalized the notion of independent ZK-EDBs, which prevents adversaries from correlating their committed databases to those of honest committers. In the plain model, Chase and Visconti [7] considered zero-knowledge protocols providing stronger simulation-based security at the expense of an interactive commitment phase.

The aforementioned constructions all relate to elementary databases. Ghosh et al. 11] formalized the notion of zero-knowledge lists. In the random oracle model, they gave size-hiding protocols where the prover can demonstrate the order in which elements appear in a committed list. Goyal et al. [13] gave blackbox constructions of size-hiding database commitments supporting more general queries. Their goal is orthogonal to ours as they rely on the "MPC-in-the-head" technique [14] to obtain black-box constructions using interaction. Here, we aim at non-interactive constructions in the standard model from standard assumptions, although we restrict ourselves to range queries.

We also mention a large body of work devoted to authenticated data structures 26]32 30|29]27/12. We insist that these result address a different problem than ours as they stand in the three party setting. Namely, in order to achieve a better efficiency, they assume that the committer is a honest database owner that always faithfully computes commitments whereas proofs are generated by an untrusted server. While reasonable in some applications (e.g., certificate revocation with a trusted certification authority [26]), the assumption of a honest committer is too much to ask for in other settings. With a pricing database, for example, it is desirable to have guarantees against price discrimination by the database owner. For this reason, we focus on the two-party setting which is usually more challenging and results in less efficient schemes. Our protocols are indeed less efficient than the range queries of Ghosh et al. [12 - which, to 
our knowledge, is the best size-hiding construction handling range queries in the three-party setting - but they do not assume a trusted committer.

\section{Preliminaries}

Notations. In our notations, $\lambda$ always stands for the security parameter. Let $\epsilon$ denote the empty string. For $x \in\{0,1\}^{\ell}$, let $x^{\prime}$ be the binary string that is equal to $x$ except with the final bit flipped and $x 0$ be ( $x 1$ respectively) the string of length $\ell+1$ with 0 ( 1 respectively) appended to $x$. Besides that, we denote the string consisting of the first $i$ bits of $x$ with $\left.x\right|_{i}$. For a string of length $\ell,\left.x\right|_{0}=\epsilon$ and $\left.x\right|_{\ell}=x$. For a set $\mathcal{S}, U(\mathcal{S})$ denotes the uniform distribution over $\mathcal{S}$ and $x \leftarrow U(\mathcal{S})$ means that element $x$ is sampled from the distribution $U(\mathcal{S})$.

For another elementary database $\mathrm{D}=\{(x, \mathrm{D}(x))\} \subset\left[0,2^{\ell}\right) \times\left[0,2^{\ell}\right)$, a set of key-value pairs, let $[\mathrm{D}]$ denote the set of keys $x \in\left[0,2^{\ell}\right)$ such that there exists a $y \in\left[0,2^{\ell}\right)$ with $(x, y) \in \mathrm{D}$. We write $\mathrm{D}(x)=\perp$ to indicate that there exists no $y \in\left[0,2^{\ell}\right)$ such that $(x, y) \in \mathrm{D}$. We write $x \in \mathrm{D}$ to say that $(x, \mathrm{D}(x)) \in \mathrm{D}$ for some $\mathrm{D}(x) \in\left[0,2^{\ell}\right)$, if there is no ambiguity. For a range $\mathfrak{R}=\left[a_{x}, b_{x}\right] \times\left[a_{y}, b_{y}\right]$, we use $[\Re]$ to denote $\left[a_{x}, b_{x}\right]$.

\subsection{Trapdoor Mercurial Commitments}

Informally, trapdoor mercurial commitments (TMC) are commitment schemes with two flavors of commitments and openings: hard and soft. Hard commitments are like regular commitments to a message $M$ and can only be hardand soft-opened to $M$. Hard openings are like regular openings for hard commitments. Soft commitments commit to no particular message and cannot be hard-opened at all but can be soft-opened to any message. Soft openings tease that a commitment potentially opens to some message $M$, and corresponds to the statement "if this commitment can be hard-opened at all, it can only be to $M$ ". Following the definitions proposed by Catalano, Dodis and Visconti [2], TMC

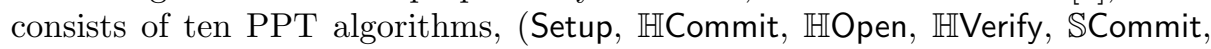
SOpen, SVerify, MFake, HEquivocate, SEquivocate).

$-(m p k, m s k) \leftarrow \operatorname{Setup}\left(1^{\lambda}\right)$ : Taking security parameter $\lambda$ as input, outputs a public mercurial commitment key $m p k$ and secret mercurial trapdoor $m s k$.

$-C \leftarrow \mathbb{H}$ Commit $(m p k, M ; R)$ : Taking public key $m p k$, message $M$ and random coins $R$ as inputs, outputs a hard commitment $C$ for $M$.

$-\pi \leftarrow \mathbb{H}$ Open $(m p k, M ; R)$ : Taking public key $m p k$, message $M$ and random coins $R$ as inputs, outputs a hard opening $\pi$ for $C$ of $M$.

- $\mathbb{H}$ Verify $(m p k, M, C, \pi)$ : Taking public key $m p k$, message $M$, commitment $C$ and hard opening $\pi$ as inputs, accepts if $\pi$ proves that $C$ is a valid hard commitment to $M$ and rejects otherwise.

$-C \leftarrow \mathbb{S}$ Commit $(m p k ; R)$ : Taking public key $m p k$ and random coins $R$ as inputs, output a soft commitment $C$ to no message in particular. 
$-\tau \leftarrow \mathbb{S}$ Open $(m p k, M$, flag; $R)$ : Given $m p k, M$, a flag flag and random coins $R$, if flag $=\mathbb{H}$, output soft opening $\tau$ "associated" to hard commitment $C=\mathbb{H}$ Commit $(m p k, M ; R)$. Otherwise, flag $=\mathbb{S}$ and $\tau$ is a soft opening "associated" to the soft commitment $C=\mathbb{S C o m m i t}(m p k ; R)$ for message $M$.

- SVerify $(m p k, M, C, \tau)$ : Taking public key $m p k$, message $M$, commitment $C$ and soft opening $\tau$, accepts if $C$ can be potentially hard opened to $M$ in the future and rejects otherwise.

$-C \leftarrow \operatorname{MFake}(m s k ; R)$ : Taking secret key $m s k$ and random coins $R$ as inputs, outputs a "fake" commitment $C$ that are initially not tied to any message.

$-\pi \leftarrow \mathbb{H}$ Equivocate $(m s k, M ; R)$ : Taking secret key $m s k$, message $M$ and random coins $R$, outputs a supposedly valid hard opening $\pi$ (hard-fake) of the fake commitment $C=\operatorname{MFake}(m s k ; R)$ to $M$.

- $\tau \leftarrow$ SEquivocate $(m s k, M ; R)$ : Taking secret key $m s k$, message $M$ and random coins $R$, outputs a supposedly valid soft opening $\tau$ (soft-fake) of the fake commitment $C=\operatorname{MFake}(m s k ; R)$.

Remark 1. In many cases, including all currently known constructions, the soft opening of a hard commitment is a proper part of the hard opening to the same message. Therefore, $\mathbb{S}$ Verify performs a proper subset of the tests done by $\mathbb{H} V$ erify. Such trapdoor mercurial commitment schemes are called proper.

Correctness. Trapdoor mercurial commitments are correct if, with overwhelming probability, for all $(m p k, m s k) \leftarrow \operatorname{Setup}\left(1^{\lambda}\right)$, and message space $\mathcal{M}$

- Hard commitments: For all messages $M \in \mathcal{M}$ and for all random coins $R$, if $C=\mathbb{H}$ Commit $(m p k, M ; R)$, then

1. for all $\tau \leftarrow \mathbb{S O p e n}(m p k, M, \mathbb{H} ; R)$, $\mathbb{S} \operatorname{Verify}(m p k, M, C, \tau)$ accepts.

2. for all $\pi \leftarrow \mathbb{H O p e n}(m p k, M ; R)$, $\mathbb{H} V$ erify $(m p k, M, C, \pi)$ accepts.

- Soft commitments: For all coins $R$, if $C \leftarrow \mathbb{S}$ Commit $(m p k ; R)$, then for all $M \in \mathcal{M}$ and $\tau \leftarrow \mathbb{S}$ Open $(m p k, M, \mathbb{S} ; R)$, SVerify $(m p k, M, C, \tau)$ accepts.

- Equivocations: For all random coins $R$, if $C \leftarrow \operatorname{MFake}(m s k ; R)$, then for all $M \in \mathcal{M}$, the following conditions are satisfied w.h.p.

1. If $\pi \leftarrow \mathbb{H}$ Equivocate $(m s k, M ; R)$, $\mathbb{H} V \operatorname{erify}(m p k, M, C, \pi)$ accepts.

2. If $\tau \leftarrow \mathbb{S}$ Equivocate $(m s k, M ; R)$, SVerify $(m p k, M, C, \tau)$ accepts.

3. If $R^{\prime} \leftarrow$ FakeExplain $(m s k, R)$, we have $C=\mathbb{S C o m m i t}\left(m p k ; R^{\prime}\right)$.

Security. The security properties are similar to trapdoor commitments, binding, hiding and equivocation, except they are modified to accommodate the two different flavors of commitments and openings.

- Mercurial-binding: Given $m p k$, no PPT adversary $\mathcal{A}$ can find $C, M, \pi, M^{\prime}, \pi^{\prime}$ (respectively $C, M, \tau, M^{\prime}, \pi^{\prime}$ ) such that $\pi$ (respectively $\tau$ ) is a valid hard (respectively soft) opening of $C$ to $M$ and $\pi^{\prime}$ is a valid hard opening of $C$ to $M \neq M^{\prime}$.

- Mercurial-hiding: No PPT adversary $\mathcal{A}$, given $m p k$, can find a message $M \in$ $\mathcal{M}$ where it can distinguish a random hard commitment/soft opening tuple $(M, \mathbb{H}$ Commit $(m p k, M ; R)$, SOpen $(m p k, M, \mathbb{H} ; R))$ from a random soft commitment/soft opening tuple $(M, \mathbb{S} C o m m i t(m p k ; R)$, SOpen $(m p k, M, \mathbb{S} ; R))$. 
In particular, the mercurial-binding property implies that $\mathcal{A}$ cannot find $C$ which can be soft-opened or hard-opened to one message and then hard-opened to another: a soft opening can never disagree with a hard opening. This also implies the infeasibility of hard opening a commitment $C$ to some message and simultaneously explain it as a soft commitment.

Catalano et al. 2] formalized the hiding properties of trapdoor mercurial commitments with several equivocation properties. They require the existence of an algorithm producing fake commitments which can be equivocated in a hard and soft way using a trapdoor. Even if the trapdoor is public, it should be infeasible to distinguish fake commitments and their equivocations into hard (resp. soft) commitments from hard (resp. soft) commitments and their hard (resp. soft) openings. On top of these three equivocation properties, we introduce a 4-th property called Soft-Explain equivocation (or SE equivocation for short). Namely, the trapdoor $m s k$ should make it possible to explain a fake commitment by outputting plausible random coins that explain it as a soft commitment.

- Equivocation: There are three related conditions for equivocation that have to be satisfied by mercurial commitments. Each is defined by a pair of games, one real and one ideal, and no PPT adversary A can distinguish between them, even if the trapdoor key $m s k$ is given at the beginning of each game, real or ideal. In all games $R$ denotes a set of random coins sampled from the appropriate distribution.

- HH Equivocation: The real game has $\mathcal{A}$ choose a message $M \in \mathcal{M}$ and receive $(M, \mathbb{H}$ Commit $(m p k, M ; R), \mathbb{H}$ Open $(m p k, M ; R))$ while the ideal game has $\mathcal{A}$ choose a message $M \in \mathcal{M}$ and obtain the tuple $(M, \operatorname{MFake}(m s k ; R), \mathbb{H}$ Equivocate $(m s k, M ; R))$.

- HS Equivocation: The real game has $\mathcal{A}$ choose a message $M \in \mathcal{M}$ and receive $(M, \mathbb{H}$ Commit $(m p k, M ; R), \operatorname{SOpen}(m p k, M ; R))$ while the ideal game has $\mathcal{A}$ choose a message $M \in \mathcal{M}$ and obtain the tuple $(M, \operatorname{MFake}(m s k ; R)$, SEquivocate $(m s k, M ; R))$.

- $S S$ Equivocation: The real game has $\mathcal{A}$ first get $C=\mathbb{S} C o m m i t(m p k ; R)$, then choose $M \in \mathcal{M}$ and finally receive $\mathbb{S O p e n}(m p k, M, \mathbb{S} ; R)$ while the ideal game has $\mathcal{A}$ first get $C=\operatorname{MFake}(m s k ; R)$, then choose $M \in \mathcal{M}$ and receive $\mathbb{S E q u i v o c a t e}(m s k, M ; R)$.

Remark 2. As noted by Catalano et al. 2, HS and SS equivocation implies mercurial-hiding. In addition, for proper mercurial commitments, HH equivocation implies HS equivocation. So it suffices to verify HH and SS equivocations and mercurial-binding for the security of any proper mercurial commitment scheme.

\subsection{Merkle Trees}

Let $\mathcal{T}_{\ell}$ denote a full and complete binary tree of depth $\ell$, with the depth of the root defined as 0 and leaves $\ell$. Nodes at depth $i>0$ are labeled with $i$-bit binary strings corresponding to the $i$-bit binary decomposition of 0 to $2^{i}-1$. Let $[a, b],[a, b)$ denote the set $\{a, a+1, \ldots, b-1, b\}$ and $\{a, a+1, \ldots, b-1\}$ 
respectively. For any node $x$ in the tree $\mathcal{T}_{\ell}$, we let $x^{\prime}$ mean its sibling in the tree. We call the canonical covering of $[a, b], \mathcal{P}_{[a, b]}$, the unique minimal set of nodes of $\mathcal{T}_{\ell}$ such that each node in $[a, b]$ is the descendant of some node in $\mathcal{P}_{[a, b]}$ and for every node in $x \in \mathcal{P}_{[a, b]}$, the subtree rooted at $x$ has leaves that are all within $[a, b]$.

Zero-Knowledge Elementary Databases and Sets. Proposed by Micali, Rabin and Kilian [21, zero-knowledge elementary databases (ZK-EDB) and sets (ZKS) enable efficient answers to membership queries in zero-knowldege. ZK-EDB is a scheme that allows one to commit to a secret database D of records and non-interactively produce proofs of (non-)membership. Membership queries on $\mathrm{D}$ committed in $\operatorname{com}_{\mathrm{D}}$ take a key $x$ as input and expect an answer $(x, \mathrm{D}(x))$ which is the record in $\mathrm{D}$ corresponding to the key $x$ if $x \in \mathrm{D}$. In particular, zero-knowledge sets are ZK-EDBs where $\mathrm{D}(x)=1$ if $x \in \mathrm{D}$.

Formally, a ZK-EDB has four algorithms (Init, ComDB, ProveQ, VerifyQ),

$-(c r s, t k) \leftarrow \operatorname{Init}\left(1^{\lambda}\right)$ : Taking security parameter $\lambda$ as input, generates and outputs common reference string (CRS) $c r s$ and trapdoor information $t k$.

- $($ com,$\Delta) \leftarrow \operatorname{ComDB}(c r s, \mathrm{D})$ : Taking the CRS $c r s$ and database D as inputs, outputs a commitment of $\mathrm{D}, \mathrm{com}$, and opening information $\Delta$.

- $\Pi_{x} \leftarrow$ ProveQ $(\mathrm{crs},(\mathrm{com}, \Delta), x)$ : Taking the CRS $\mathrm{crs}$, database commitment and opening information (com, $\Delta$ ) and key $x$ as inputs, outputs a proof $\Pi_{x}$ of either $x \in \mathrm{D}$ or $x \notin \mathrm{D}$.

$-y \leftarrow \operatorname{Verify} Q\left(\operatorname{crs}, \operatorname{com}, x, \Pi_{x}\right)$ : Taking the CRS crs, database commitment com, key $x$ and proof $\Pi_{x}$ as inputs, outputs $y$ where

$$
y= \begin{cases}\mathrm{D}(x), & \text { if } x \in[\mathrm{D}] \\ \perp, & \text { if } x \notin[\mathrm{D}] \\ b a d, & \text { if it otherwise believes that the prover is cheating. }\end{cases}
$$

Security. The three security properties of ZK-EDB are completeness, soundness and zero-knowledge. The first one requires that honestly generated proofs always satisfy verification with VerifyQ. Soundness mandates that provers be unable to produce a key $x$ and successful proofs $\Pi_{x}, \Pi_{x}^{\prime}$ such that they do not verify to the same value $y$. Finally, zero-knowledge implies that each proof $\Pi_{x}$ only reveals the value $\mathrm{D}(x)$ and nothing else about $\mathrm{D}$.

Merkle Trees from Trapdoor Mercurial Commitments. Although Micali et al. constructed ZKS and ZK-EDB specifically from number-theoretic assumptions, Chase et al. [5]6] introduced the TMC primitive and showed that ZKS and ZK-EDB are simply Merkle trees built with TMC. The key to their size-hiding property is that TMC allows a committer compute portions of the Merkle tree that do not contain database elements only when required in proofs.

We detail four algorithms, BuildTree, HOpenPath, SOpenPath and VerifyPath, which we will use in Sections 3, 4. These algorithms encapsulate the construction of a ZK-EDB scheme from TMC in [56]: ComDB corresponds to BuildTree, ProveQ to $\mathbb{H}$ OpenPath and $\mathbb{S} O p e n P a t h$ based on the value $\mathrm{D}(x)$ and VerifyQ to 
VerifyPath. Let $\lambda$ be a security parameter, $(c r s, t k) \leftarrow \operatorname{Setup}\left(1^{\lambda}\right)$ and a database $\mathrm{D}=\left\{(x, \mathrm{D}(x)) \mid(x, \mathrm{D}(x)) \in\left[0,2^{\ell}\right) \times\left[0,2^{\ell}\right)\right\}$.

- $($ com,$\Delta) \leftarrow$ BuildTree $($ crs, D): Taking as inputs CRS crs and database D, build a Merkle tree of depth $\ell$, indexed by strings in $\bigcup_{i=0}^{\ell}\{0,1\}^{i}$, as follows:

1. For each leaf $j \in\{0,1\}^{\ell}$ with $\mathrm{D}(j) \neq \perp, C_{j}=\mathbb{H}$ Commit $\left(c r s, \mathrm{D}(j) ; R_{j}\right)$. For every leaf $j$ with its sibling $j^{\prime} \in \mathrm{D}$ but $j \notin \mathrm{D}$, set $C_{j}=\mathbb{S C o m m i t}\left(\operatorname{crs} ; R_{j}\right)$. For all other leaves $j$, set $C_{j}=n i l$.

2. At depth $i$ from $\ell-1$ to 0 and each $\rho \in\{0,1\}^{i}$, define $C_{\rho}$ as follows. For all $\rho$ such that $C_{\rho 0}, C_{\rho 1} \neq$ nil, set $C_{\rho}=\mathbb{H}$ Commit $\left(\operatorname{crs},\left(C_{\rho 0}, C_{\rho 1}\right) ; R_{\rho}\right)$. For all $\rho$ such that $C_{\rho}$ was defined but not $C_{\rho^{\prime}}, C_{\rho^{\prime}}=\mathbb{S C o m m i t}\left(\right.$ crs $\left.; R_{\rho^{\prime}}\right)$. For any other string $\rho \in\{0,1\}^{i}$, set $C_{\rho}=n i l$.

3. After the end of Step 2, if the value at the root $C_{\epsilon}=$ nil, meaning $\left\{C_{j}\right\}=\emptyset$, then set $C_{\epsilon}=\mathbb{S}$ Commit $\left(\right.$ crs $\left.; R_{\epsilon}\right)$.

Output com $=C_{\epsilon}$ and $\Delta=\left\{R_{j}\right\}$, the set of random coins for all commitments computed in the steps above.

- $\Pi_{z} \leftarrow \mathbb{H}$ OpenPath $($ crs $,($ com,$\Delta), z)$ : Given crs, a database commitment com and the opening information $\Delta$ for a database $\mathrm{D}$ and a key $z \in \mathrm{D}$, define the hard authentication path for $z \in \mathrm{D}$ as the set of hard openings for nodes in indices $z=\left.z\right|_{\ell},\left.z\right|_{\ell-1}, \ldots,\left.z\right|_{1}$ which form a path from $z$ to the root $\epsilon=\left.z\right|_{0}$. Proceed to decommit all the nodes on the path as follows:

1. Compute $\pi_{z} \leftarrow \mathbb{H} O \operatorname{pen}\left(\operatorname{crs},(z, \mathrm{D}(z)) ; R_{z}\right)$.

2. At each depth $j$ from $\ell-1$ to 0 , compute the hard opening for $C_{\left.z\right|_{j}}$ to $\left(C_{\left.z\right|_{j} 0}, C_{\left.z\right|_{j} 1}\right), \pi_{\left.z\right|_{j}} \leftarrow \mathbb{H} O \operatorname{pen}\left(\right.$ crs,$\left.\left(C_{\left.z\right|_{j} 0}, C_{\left.z\right|_{j} 1}\right) ; R_{\left.z\right|_{j}}\right)$.

Output $\Pi_{z}=\left(\mathrm{D}(z),\left\{C_{\left.z\right|_{j}}, C_{\left(\left.z\right|_{j}\right)^{\prime}}\right\}_{1 \leq j \leq \ell},\left\{\pi_{\left.z\right|_{j}}\right\}_{0 \leq j \leq \ell}\right)$.

- $\Pi_{z} \leftarrow$ SOpenPath $(\mathrm{crs},(\mathrm{com}, \Delta), z)$ : Taking as inputs CRS crs, database commitment com and opening information $\Delta$ for a database $\mathrm{D}$ and a key $z \in \mathrm{D}$, define the soft authentication path for $z \notin \mathrm{D}$ as the set of soft openings for nodes at indices $z=\left.z\right|_{\ell},\left.z\right|_{\ell-1}, \ldots,\left.z\right|_{1}$ which form a path from $z$ to the root $\epsilon=\left.z\right|_{0}$. Let $h$ be the largest value such that $C_{\left.z\right|_{h}} \neq n i l$.

1. If the complete path does not exist, i.e., $C_{z}=n i l$, fill it out to leaf $z$ :

a. Compute $C_{z}=\operatorname{SCommit}\left(\right.$ crs $\left.; R_{z}\right), C_{z^{\prime}}=\operatorname{SCommit}\left(\mathrm{crs} ; R_{z^{\prime}}\right)$.

b. At depth $j$ from $\ell-1$ to $h+1$, compute $C_{\left.z\right|_{j}}=\operatorname{SCommit}\left(\right.$ crs $\left.; R_{\left.z\right|_{j}}\right)$ and $C_{(z \mid j)^{\prime}}=\mathbb{S C o m m i t}\left(\operatorname{crs} ; R_{\left(\left.z\right|_{j}\right)^{\prime}}\right)$.

2. Otherwise, $C_{z}=\mathbb{S C o m m i t}\left(c r s ; R_{z}\right)$ and we proceed to the next step.

3. Produce soft openings to nodes along the path from leaf $z$ to the root.

a. Compute $\tau_{z}=\mathbb{S O p e n}\left(\operatorname{crs}, \perp, \mathbb{S} ; R_{z}\right)$, soft opening of $C_{z}$ to $\perp$.

b. At depth $j$ from $\ell-1$ to $h+1$, compute soft openings of $C_{\left.z\right|_{j}}$ to their children, $\tau_{\left.z\right|_{j}}=\mathbb{S O p e n}\left(\operatorname{crs},\left(C_{\left.z\right|_{j} 0}, C_{\left.z\right|_{j} 1}\right), \mathbb{S} ; R_{\left.z\right|_{j}}\right)$.

c. For $j$ from $h$ to 1 , compute $\tau_{\left.z\right|_{h}}=\mathbb{S O p e n}\left(\operatorname{crs},\left(C_{\left.z\right|_{h} 0}, C_{\left.z\right|_{h} 1}\right), \mathbb{H} ; R_{\left.z\right|_{h}}\right)$.

d. If $C_{\epsilon}=\mathbb{S}$ Commit $\left(\right.$ crs $\left.; R_{\epsilon}\right)$, set $\tau_{\epsilon}=\mathbb{S O p e n}\left(\right.$ crs $\left.,\left(C_{\left.z\right|_{0}}, C_{\left.z\right|_{1}}\right), \mathbb{S} ; R_{\epsilon}\right)$. Otherwise, $\tau_{\epsilon}=\mathbb{S O p e n}\left(\operatorname{crs},\left(C_{\left.z\right|_{0}}, C_{\left.z\right|_{1}}\right), \mathbb{H} ; R_{\epsilon}\right)$ 
Output $\Pi_{z}=\left(\perp,\left\{C_{\left.z\right|_{j}}, C_{\left(\left.z\right|_{j}\right)^{\prime}}\right\}_{1 \leq j \leq \ell},\left\{\tau_{\left.z\right|_{j}}\right\}_{0 \leq j \leq \ell)}\right)$. Also, add any random coins used when a path is filled out to $\Delta$ for use with later proofs.

- ans $\leftarrow$ VerifyPath $\left(c r s, c o m, z, \Pi_{z}\right)$ : Taking as inputs CRS crs, database commitment com, key $z$ and proof $\Pi_{z}$, check the proof which has two possible forms:

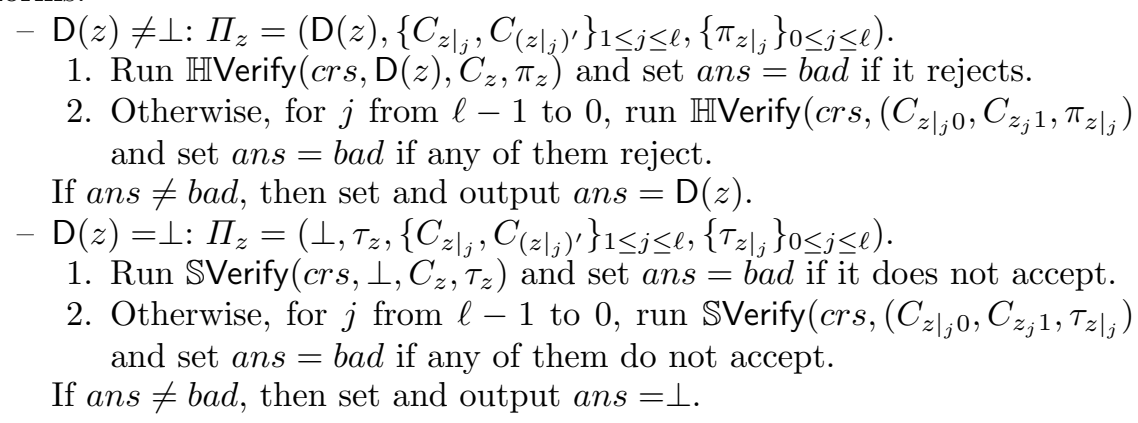

Complete Subtree Method. We recall the complete subtree method proposed by Naor, Naor and Lotspiech [25], which is one of the algorithms in the subsetcover framework. This technique is also used by Ghosh et al. [12] to obtain onedimensional range queries in the three party setting. This work, on the other hand, is in the two party setting which is more challenging to realize.

For a full and complete binary tree of depth $\ell, \mathcal{T}_{\ell}$, with nodes indexed by binary strings of length up to $\ell$. Every node $x$ of $\mathcal{T}_{\ell}$ defines a subset $\mathcal{S}_{x}$ of leaves, those in the full and complete subtree rooted at $x$. Conversely, for a given set of leaves $\mathcal{R}$, a directed Steiner Tree, denoted by $S T(\mathcal{R})$ in $\mathcal{T}_{\ell}$, is induced. $S T(\mathcal{R})$ is the minimal subtree (rooted at $\epsilon$ ) of $\mathcal{T}_{\ell}$ that connects all the leaves in $\mathcal{R}$. Let $\mathcal{P}=\left\{p_{1}, \ldots, p_{m}\right\}$ be the set of nodes that are adjacent to nodes of outdegree one in $S T(\mathcal{R})$, which is the canonical covering of $\left[0,2^{\ell}\right) \backslash \mathcal{R}$. Naor, Naor and Lotspiech [25] found that the size of $\mathcal{P}$ is upper-bounded by $|\mathcal{R}| \log \left(2^{\ell} /|\mathcal{R}|\right)$.

\subsection{Background on Lattices}

Lattices. Let $n, m$, and $q \geq 2$ be integers. For matrix $\mathbf{A} \in \mathbb{Z}_{q}^{n \times m}$, define the $m$-dimensional lattice:

$$
\Lambda^{\perp}(\mathbf{A})=\left\{\mathbf{x} \in \mathbb{Z}^{m}: \mathbf{A} \cdot \mathbf{x}=\mathbf{0} \bmod q\right\} \subseteq \mathbb{Z}^{m} .
$$

For any $\mathbf{u}$ in the image of $\mathbf{A}$, define $\Lambda^{\mathbf{u}}(\mathbf{A})=\left\{\mathbf{x} \in \mathbb{Z}^{m}: \mathbf{A} \cdot \mathbf{x}=\mathbf{u} \bmod q\right\}$.

Definition $1\left(\mathrm{SIS}_{n, m, q, \beta}\right)$. Given a uniformly random matrix $\mathbf{A} \in \mathbb{Z}_{q}^{n \times m}$, find a non-zero vector $\mathbf{v} \in \Lambda^{\perp}(\mathbf{A})$ such that $\|\mathbf{v}\| \leq \beta$.

If $m, \beta \in \operatorname{poly}(n)$ and $q>\beta \cdot \omega(\sqrt{n \log n})$, then the $\operatorname{SIS}_{n, m, q, \beta}$ problem is at least as hard as lattice problem $\operatorname{SIVP}_{\gamma}$ for some $\gamma=\beta \cdot \widetilde{\mathcal{O}}(\sqrt{n})$ (see, e.g., [10[23).

Gaussian distributions. For integer $m>0$, let $D_{\mathbb{Z}^{m}, \sigma}$ be the discrete Gaussian distribution over $\mathbb{Z}^{m}$ with parameter $\sigma>0$. In the following lemmas, we review several well-known facts from [10]. 
Lemma 1. We have $\operatorname{Pr}\left[\|\mathbf{r}\|>\sigma \sqrt{m} \mid \mathbf{r} \hookleftarrow D_{\mathbb{Z}^{m}, \sigma}\right] \leq 2^{-m}$.

Lemma 2. Let $n$ be a positive integer, $q$ be a prime, $m \geq 2 n \log q$ and $\sigma=$ $\Omega(\sqrt{n \log q \log n})$. Then, for a uniformly random $\mathbf{A} \in \mathbb{Z}_{q}^{n \times m}$ and for $\mathbf{r} \hookleftarrow D_{\mathbb{Z}^{m}, \sigma}$, the distribution of $\mathbf{u}=\mathbf{A} \cdot \mathbf{r} \bmod q$ is statistically close to uniform over $\mathbb{Z}_{q}^{n}$. Moreover, the conditional distribution of $\mathbf{r}$ given $\mathbf{u}$ is $D_{\Lambda^{\mathbf{u}}(\mathbf{A}), \sigma}$.

Lemma 3. For $\sigma \geq \widetilde{\mathcal{O}}(\sqrt{m})$, the min-entropy of $D_{\mathbb{Z}^{m}, \sigma}$ is at least $m-1$.

When sampling a matrix $\mathbf{R}=\left[\mathbf{r}_{1}|\cdots| \mathbf{r}_{w}\right] \in \mathbb{Z}^{m \times w}$, where $\mathbf{r}_{i} \hookleftarrow D_{\mathbb{Z}^{m}, \sigma}$ for all $i=1, \ldots, w$, we will use the notation $\mathbf{R} \hookleftarrow D_{\mathbb{Z}^{m \times w}, \sigma}$.

Trapdoors for Lattices. We will employ the lattice trapdoors introduced by Micciancio and Peikert 22. For any positive integer $k$, let $\mathbf{I}_{k}$ denote the identity matrix of order $k$. Let $n$ be a positive integer, $q \in \operatorname{poly}(n)$ be a modulus and $w=n\lceil\log q\rceil$. Define the gadget matrix $\mathbf{G}=\mathbf{I}_{n} \otimes\left(1,2, \ldots, 2^{\lceil\log q\rceil-1}\right) \in \mathbb{Z}_{q}^{n \times w}$.

Let $m=\bar{m}+w$, for some $\bar{m}>w$. A trapdoor for matrix $\mathbf{A} \in \mathbb{Z}_{q}^{n \times m}$ is a matrix $\mathbf{R} \in \mathbb{Z}^{\bar{m} \times w}$ such that $\mathbf{A}\left[\begin{array}{c}\mathbf{R} \\ \mathbf{I}_{w}\end{array}\right]=\mathbf{G}$. In particular, if $\mathbf{A}=[\overline{\mathbf{A}} \mid \mathbf{G}-\overline{\mathbf{A}} \cdot \mathbf{R}]$, where $\overline{\mathbf{A}} \in \mathbb{Z}_{q}^{n \times \bar{m}}$, then $\mathbf{R}$ is a trapdoor for $\mathbf{A}$.

Lemma 4 ([22] ). Let $n, q, w, \bar{m}, m$ be as above. Then, there exists a PPT algorithm $\operatorname{TrapGen}(n, m, q)$ that outputs a matrix $\mathbf{A} \in \mathbb{Z}_{q}^{n \times m}$ together with a trapdoor $\mathbf{R} \in \mathbb{Z}^{\bar{m} \times w}$, such that the distribution of $\mathbf{A}$ is statistically close to uniform.

Moreover, for any $\mathbf{u} \in \mathbb{Z}_{q}^{n}$ and $\sigma=\Omega(\sqrt{n \log q \log n})$, there exists a PPT algorithm SampleD $(\mathbf{R}, \mathbf{A}, \mathbf{u}, \sigma)$ that outputs $\mathbf{r} \in \mathbb{Z}^{m}$ sampled from a distribution statistically close to $D_{\Lambda^{\mathrm{u}}(\mathbf{A}), \sigma}$.

As shown by Micciancio and Peikert, a trapdoor for matrix $\mathbf{A} \in \mathbb{Z}_{q}^{n \times m}$ can be efficiently extended into a trapdoor for any matrix $\mathbf{B} \in \mathbb{Z}_{q}^{n \times(m+w)}$ of the form $\mathbf{B}=\left[\mathbf{A} \mid \mathbf{A}^{\prime}\right]$, where matrix $\mathbf{A}^{\prime} \in \mathbb{Z}_{q}^{n \times w}$.

\section{Zero-Knowledge Expressive Elementary Database from Trapdoor Mercurial Commitments}

We construct a new flavor of size-hiding zero-knowledge database, called zeroknowledge expressive elementary database (ZK-EEDB). It allows databases D to be secretly committed in a public digest and several queries on $D$ to be efficiently answered in zero-knowledge. The databases supported by ZK-EEDB are sets of records, which are key-value pairs $(x, \mathrm{D}(x)) \in\left[0,2^{\ell}\right) \times\left[0,2^{\ell}\right)$ and the queries supported by ZK-EEDB include queries over keys and values.

Besides membership over keys which was previously considered by Micali, Rabin and Kilian [21] in zero-knowledge elementary database, ZK-EEDB enables range queries over records of $D$, generalizing range queries over keys and values. We introduce the ability to generate proofs of correctness for answers to range queries over values in zero-knowledge with ZK-EEDB. The membership query over values, in this work, is the query which, given $y$, asks for the set $\mathrm{D}^{-1}(y)=$ 
$\left\{x_{i} \mid x_{i} \in[\mathrm{D}]\right.$ such that $\left.\mathrm{D}\left(x_{i}\right)=y\right\}$. A range query over values is membership extended to a range of values $\left[a_{y}, b_{y}\right]$. From our techniques, we gain the ability to prove correctness of answers to range queries over records that is efficient for any super-polynomial range of keys.

First, we introduce new notations for values of a database $D$ and the query types considered in ZK-EEDB. Following that, ZK-EEDB is formally defined and its security properties detailed. Then, we describe our techniques that enable efficient range queries over records of a database D. Finally, we end the section with a construction with TMC.

A Database of Values, $D^{-1}$. In this work, we consider queries over values of a database $\mathrm{D}$ in addition to queries over its keys. To achieve it efficiently, we use an alternate view of $\mathrm{D}$, called $\mathrm{D}^{-1}$, which is essentially a "reversed directory": namely, $\mathrm{D}^{-1}$ is the set $\left\{\left(y, \mathrm{D}^{-1}(y)\right) \mid y \in\left[0,2^{\ell}\right)\right\}$, where $\mathrm{D}^{-1}(y)=\{x \mid x \in$ $\mathrm{D}$ and $\mathrm{D}(x)=y\}$. The key-space of the database $\mathrm{D}^{-1}$ is thus the value-space of $\mathrm{D}$ and each key $y \in\left[\mathrm{D}^{-1}\right]$ has a value $\mathrm{D}^{-1}(y)$, which is the set of keys $x \in[\mathrm{D}]$ that are assigned the value $y$ (i.e., $\mathrm{D}(x)=y$ ).

Queries in ZK-EEDB. Note that the answer to any query should come with a proof of correctness. We now describe a specific kind of query supported by our ZK-EEDB primitive which actually captures a total of six different queries.

- Range (Record) queries: Given a range $\mathfrak{R}=\left[a_{x}, b_{x}\right] \times\left[a_{y}, b_{y}\right] \subset\left[0,2^{\ell}\right) \times\left[0,2^{\ell}\right)$, they return the set $\mathcal{L}$ of records such that $\mathcal{L}=\mathrm{D} \cap\left(\left[a_{x}, b_{x}\right] \times\left[a_{y}, b_{y}\right]\right)$.

- For general $\mathfrak{R}=\left[a_{x}, b_{x}\right] \times\left[a_{y}, b_{y}\right],\left[a_{x}, b_{x}\right]$ can be super-polynomial in $\ell$.

- Range queries over values (resp. keys) correspond to the input range $\left[0,2^{\ell}\right) \times\left[a_{y}, b_{y}\right]\left(\right.$ resp. $\left.\left[a_{x}, b_{x}\right] \times\left[0,2^{\ell}\right)\right)$. For such queries, the interval $\left[a_{y}, b_{y}\right]$ (resp. $\left.\left[a_{x}, b_{x}\right]\right)$ can be super-polynomial or even exponential in $\ell$.

- Membership queries over records (resp. values and keys) correspond to the input range $[x, x] \times[y, y]\left(\operatorname{resp} .\left[0,2^{\ell}\right) \times[y, y]\right.$ and $\left.[x, x] \times\left[0,2^{\ell}\right)\right)$.

\subsection{Zero-Knowledge Expressive Elementary Database}

ZK-EEDB has four algorithms: Init, ComDB, ProveRQ, VerifyRQ.

$-($ crs,$t k) \leftarrow \operatorname{Init}\left(1^{\lambda}\right)$ : Takes as input security parameter $\lambda$ and outputs a common reference string (CRS) crs and trapdoor key $t k$.

$-($ com,$\Delta) \leftarrow \operatorname{ComDB}($ crs, $\mathrm{D})$ : Takes in crs and a database $\mathrm{D}=\{(x, \mathrm{D}(x))\}$. It returns a commitment com to $\mathrm{D}$ and a decommitment information $\Delta$.

- $\Pi_{\mathfrak{R}} \leftarrow \operatorname{ProveRQ}($ crs $,($ com, $\Delta), \mathfrak{R})$ : Inputs crs, a database commitment and decommitment information $($ com, $\Delta)$ and a range $\mathfrak{R}$. It returns a proof of correctness $\Pi_{\mathfrak{R}}$ of the range query with input range $\mathfrak{R} \subset\left[0,2^{\ell}\right) \times\left[0,2^{\ell}\right)$.

$-\mathcal{L} \leftarrow \operatorname{VerifyRQ}\left(\right.$ crs, com, $\left.\mathfrak{R}, \Pi_{\mathfrak{R}}\right)$ : Inputs crs, a database commitment com, a range $\mathfrak{R} \subset\left[0,2^{\ell}\right) \times\left[0,2^{\ell}\right)$ and a purported proof $\Pi_{\mathfrak{R}}$. It returns

$$
z= \begin{cases}\mathrm{D} \cap \mathfrak{R}, & \text { if the proof is correct; } \\ \text { bad, } & \text { if the proof is deemed invalid. }\end{cases}
$$


We consider the same properties as in standard ZK-EDB protocols: namely, completeness, soundness and zero-knowledge, adapted to support the more expressive queries in ZK-EEDB. Correctness mandates that, for any query, correctly computed proofs satisfy the verification algorithm. Zero-knowledge requires that there exist an efficient simulator which is only granted oracle access to the database and outputs proofs for queries that are indistinguishable from those produced by a real prover using the real database as a witness. Soundness requires that no contradictory statements about the committed database can be proven by the adversary. Informally speaking, no PPT adversary can find two ranges $\mathfrak{R}, \mathfrak{R}^{\prime}$ and proofs $\Pi, \Pi^{\prime}$ for which there exists a record $(x, y) \in \mathfrak{R} \cap \mathfrak{R}^{\prime}$ that is in the answer to the first query but not the second. Formally, we have

- Completeness: For all databases D and all keys $x$, we have

$\operatorname{Pr}\left[\right.$ crs $\leftarrow \operatorname{Init}\left(1^{\lambda}\right) ;($ com,$\Delta) \leftarrow \operatorname{ComDB}($ crs, $\mathrm{D}) ;$

$\Pi_{\mathfrak{R}} \leftarrow \operatorname{ProveRQ}($ crs $,($ com,$\Delta), \mathfrak{R}) ;$

$\operatorname{VerifyRQ}\left(\right.$ crs, com, $\left.\left.\mathfrak{R}, \Pi_{\mathfrak{R}}\right) \neq b a d\right]=1-\nu(\lambda)$,

for some negligible function $\nu(\cdot)$.

- Soundness: For any PPT algorithm $\mathrm{P}^{\prime}$, the probability

$\operatorname{Pr}\left[\right.$ crs $\leftarrow \operatorname{lnit}\left(1^{\lambda}\right) ;\left(\right.$ com, $\left.\mathfrak{R}, \Pi, \mathfrak{R}^{\prime}, \Pi^{\prime}\right) \leftarrow \mathrm{P}^{\prime}($ crs $) ;$

$(\operatorname{VerifyRQ}($ crs, com $, \mathfrak{R}, \Pi)=\mathcal{L} \neq$ bad $)$

$\wedge\left(\operatorname{VerifyRQ}\left(\right.\right.$ crs, com $\left., \mathfrak{R}^{\prime}, \Pi^{\prime}\right)=\mathcal{L}^{\prime} \neq$ bad $)$

$\wedge\left(\exists(x, y) \in \mathfrak{R} \cap \mathfrak{R}^{\prime}\right.$ s.t. $\left.((x, y) \in \mathcal{L}) \wedge\left((x, y) \notin \mathcal{L}^{\prime}\right)\right]$,

is bounded by $\nu(\lambda)$, for some negligible function $\nu(\cdot)$.

- Zero-Knowledge: For any PPT adversary $\mathcal{A}$ and any efficiently computable database $\mathrm{D}$, there exists an efficient simulator consisting of a triple of algorithms (SInit, SCom, SProveQ ${ }^{\mathrm{D}}$ ) such that the outputs of the following two experiment outputs are indistinguishable:

Real experiment:

1. Let $c r s \leftarrow \operatorname{Init}\left(1^{\lambda}\right),(\mathrm{com}, \Delta) \leftarrow \operatorname{ComDB}(\mathrm{crs}, \mathrm{D})$ and $s_{0}=\Pi_{0}=\varepsilon$.

2. For $1 \leq i \leq n$, we have $\left(\mathfrak{R}_{i}, s_{i}\right) \leftarrow \mathcal{A}\left(\mathrm{crs}, \mathrm{com}, \Pi_{0}, \ldots, \Pi_{i-1}, s_{i-1}\right)$ and $\mathcal{A}$ gets a real proof $\Pi_{i}=\operatorname{ProveRQ}\left(\right.$ crs $,($ com,$\left.\Delta), \Re_{i}\right)$.

The experiment outputs $\left(\operatorname{crs}, \mathfrak{R}_{1}, \Pi_{1}, \ldots, \mathfrak{R}_{n}, \Pi_{n}\right)$.

Ideal experiment:

1. Let $\left(\mathrm{crs}^{\prime}, s t_{0}\right) \leftarrow \operatorname{SInit}\left(1^{\lambda}\right),\left(\mathrm{com}^{\prime}, s t_{1}\right) \leftarrow \mathrm{SCom}\left(s t_{0}\right)$ and $s_{0}=\Pi_{0}^{\prime}=\varepsilon$.

2. For $1 \leq i \leq n$, we have $\left(\mathfrak{R}_{i}, s_{i}\right) \leftarrow \mathcal{A}\left(\operatorname{crs}^{\prime}, \operatorname{com}^{\prime}, \Pi_{0}^{\prime}, \ldots, \Pi_{i-1}^{\prime}, s_{i-1}\right)$ and $\mathcal{A}$ gets a simulated proof $\Pi_{i}^{\prime} \leftarrow \operatorname{SProveRQ}{ }^{\mathrm{D}}\left(\operatorname{crs}^{\prime}, s t_{1}, \mathfrak{R}_{i}\right)$.

The experiment outputs $\left(\operatorname{crs}^{\prime}, \mathfrak{R}_{1}, \Pi_{1}^{\prime}, \ldots, \mathfrak{R}_{n}, \Pi_{n}^{\prime}\right)$.

In the ideal experiment, SProve $Q^{\mathrm{D}}$ is an oracle that is allowed to invoke a database oracle $D$ and receive the set of records $D \cap \mathfrak{R}$ for any range $\mathfrak{R}=$ $\left[a_{x}, b_{x}\right] \times\left[a_{y}, b_{y}\right]$ chosen by the adversary.

Here, a few comments about our security definitions are in order. We recall that, in size-hiding database commitments, the commitment must be shorter 
than the database since, otherwise, an upper bound on the database size is leaked. This naturally leads us to use statistically-hiding commitments, where we cannot properly speak of the "content" of a commitment since valid openings exist for any database. What matters is thus what the adversary is able to prove about the commitments it generates. In non-interactive size-hiding database commitments (at least under falsifiable assumptions), soundness can only be defined by preventing proofs for conflicting statements. In standard ZK-EDBs, it means one cannot prove distinct values for any key in a committed DB. For ZK-EEDBs, we extend it to range queries which are akin to batch queries. Our definition of soundness is thus adjusted to account for the answer being a set of records instead a value.

In the definitions of Ostrovsky et al. 28, soundness includes that, for any valid proofs produced by the prover, there exists a valid database compatible with the proven statements. This property is straightforward to show in our scheme and can be added to our model. Furthermore, although range queries can admit exponentially large ranges, it is still necessary to hide database size to maintain the zero-knowledge property of ZK-EEDB, which requires that verifiers leak nothing beyond the statements involved in all queries.

\subsection{A Construction of ZK-EEDB from TMC: Initialization and Commitment Generation}

In this section, we describe how to initialize a ZK-EEDB instance and commit to a database D by exploiting two size-hiding trees. Details of the proof generation and verification for ZK-EEDB queries are deferred to Section 4 .

ZK-EEDB Database Commitments from TMC. To construct ZK-EEDBs, our idea is to use two Merkle trees to commit to the database D, each in a different representation. While ordinary ZK-EDBs consist of a single Merkle tree, ZK-EEDB relies on two size-hiding trees: (i) A (key) Merkle tree of height $\ell$, which commits to a value $\mathrm{D}(x)$ at each leaf $x \in[\mathrm{D}$; ; (ii) A (value) Merkle tree also of height $\ell$ that is two-tiered: each leaf $y$ stores a commitment to the root value of a size-hiding Merkle tree that accumulates $\mathrm{D}_{y}^{-1}$. Here, $\mathrm{D}_{y}^{-1}=\{(x, 1) \mid$ $(x, y) \in \mathrm{D}\}$ is a zero-knowledge set encoded as a ZK-EDB with keys $x$ and value 1 if and only if $(x, y) \in \mathrm{D}$.

The value Merkle tree can be seen as a commitment to the reversed database $\mathrm{D}_{\text {com }}^{-1}=\left\{\left(y, \operatorname{com}_{\mathrm{D}_{y}^{-1}}\right) \mid \mathrm{D}^{-1}(y) \neq \emptyset\right\}$. Although defined differently earlier, we use $\mathrm{D}^{-1}$ from here on to denote $\mathrm{D}_{\text {com }}^{-1}$. This is the main technique enabling efficient queries over values and records: Soft-opening paths in the value Merkle tree allow us to efficiently prove statements about non-membership of a value $y$ (i.e., D contains no record of the form $(*, y)$ ). In existing single-tree-based constructions of ZK-EDBs, such queries are simply impossible to prove in zeroknowledge as each key must be separately proven to not have $\mathrm{D}(x)=y$ (which betrays the database size and is highly inefficient). However, in ZK-EEDB, the root value of the Merkle tree that accumulates $\mathrm{D}_{y}^{-1}$ is simply revealed to be 
empty by explaining the value stored at the leaf $y$ in the value Merkle tree is a soft commitment $C_{y}$ and showing a soft authentication path from $y$ to the root.

With two commitments to the same database under different representations, we need to add checks to enforce that the two Merkle trees are consistent with each other. Whenever a record is proven to be in D via $\operatorname{com}_{\mathrm{D}}$ and the first Merkle tree, the same record is also proven to be correctly committed in $\operatorname{com}_{\mathrm{D}^{-1}}$ using the second Merkle tree. This prevents malicious provers from proving contradictory statements as both commitments have to agree at any record that has been proven to be in $\mathrm{D}$. We insist that a cheating prover cannot win by using inconsistent databases in the two trees since, even by doing so, it will remain unable to prove contradictory statements without breaking the binding properties of the underlying mercurial commitment.

We now describe the initialization and commitment algorithms, Init and ComDB for ZK-EEDB from the TMC scheme, TMC.

$-($ crs,$t k) \leftarrow \operatorname{Init}\left(1^{\lambda}\right)$ : compute and return $($ crs,$t k) \leftarrow \operatorname{TMC}$.Setup $\left(1^{\lambda}\right)$.

$-($ com,$\Delta) \leftarrow \operatorname{ComDB}($ crs, $\mathrm{D})$ :

1. Compute $\left(\operatorname{com}_{\mathrm{D}}, \Delta_{\mathrm{D}}\right) \leftarrow \operatorname{BuildTree}(\operatorname{crs}, \mathrm{D})$.

2. For every $y$ with $\mathrm{D}^{-1}(y) \neq \emptyset$, compute commitments of $\mathrm{D}_{y}^{-1}$ by running $\left(\operatorname{com}_{\mathrm{D}_{y}^{-1}}, \Delta_{\mathrm{D}_{y}^{-1}}\right) \leftarrow$ BuildTree $\left(\right.$ crs, $\left.\mathrm{D}_{y}^{-1}\right)$.

3. Compute $\left(\operatorname{com}_{\mathrm{D}^{-1}}, \Delta_{\mathrm{D}^{-1}}\right)$ with BuildTree $\left(\right.$ crs, $\left.\mathrm{D}^{-1}\right)$.

Return $\left(c o m=\left(\operatorname{com}_{\mathrm{D}}, \operatorname{com}_{\mathrm{D}^{-1}}\right), \Delta=\left(\Delta_{\mathrm{D}},\left\{\operatorname{com}_{\mathrm{D}_{y}^{-1}}\right\}_{y \in\left[\mathrm{D}^{-1}\right]}, \Delta_{\mathrm{D}^{-1}}\right)\right)$.

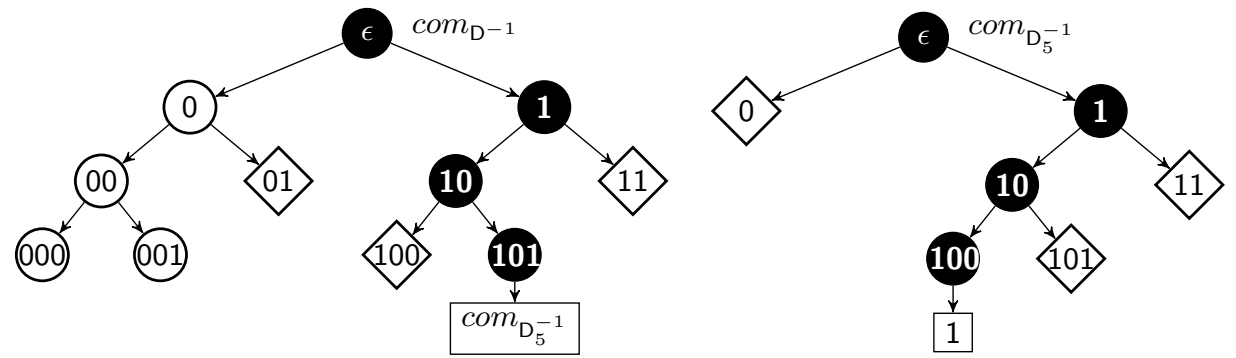

Fig. 1. The Value Merkle Tree in ZK-EEDB with Authentication Paths for $(4,5) \in \mathrm{D}$.

\section{Queries in ZK-EEDB}

We first show how to prove correctness for answers to range queries in zeroknowledge for some database D committed with a Merkle tree and TMC scheme. Then, we apply the techniques to construct the ProveRQ and VerifyRQ algorithms in ZK-EEDB. Let the TMC scheme used, TMC, be implicit in the algorithms. 


\subsection{Range Queries with A Single Merkle Tree}

For a single Merkle tree, a range query is an interval $[a, b] \subseteq\left[0,2^{\ell}\right)$ of keys. Our range query proofs uses two key ideas: Steiner trees and a set of novel explanation algorithms. We can split the leaves in $[a, b]$ into two sets, $\mathcal{R} \subseteq[\mathrm{D}]$ with values in $\mathrm{D}$, and the others, $[a, b] \backslash \mathcal{R}$. Proving correctness for $[a, b]$ means showing that every $x \in \mathcal{R}$ is a member of $[\mathrm{D}]$ and the remaining keys $[a, b] \backslash \mathcal{R}$ are not.

The Steiner tree characterizes the minimum set of nodes that have to be hard-opened to form the authentication paths for every leaf in $\mathcal{R}$. At the same time, it defines a polynomial-sized covering set for the remaining keys $[a, b] \backslash \mathcal{R}$. Then, the explanation algorithms are used to reveal that the covering set consists of soft commitments, so no hard authentication paths can be built from leaves in $[a, b] \backslash \mathcal{R}$ to the root of the Merkle tree.

Explanations for Trapdoor Mercurial Commitments. For our purposes, we introduce three new algorithms Explain, EVerify, FakeExplain to the syntax of TMC schemes. These algorithms reveal and verify that a commitment is a soft commitment and produce a "fake" proof that a fake commitment is a soft commitment. Explain is used by the prover when producing range proofs, EVerify is used by the verifier when checking if proofs are correct and FakeExplain is used by the simulator from the zero-knowledge property.

Note that Catalano et al. 22 's construction of TMCs, and thus all known TMC schemes, can be easily adapted to support the three new algorithms introduced in this work. We show this in Appendix A due to space constraints.

$-R \leftarrow \operatorname{Explain}(m p k ; R)$ : On input of the public commitment key mpk and random coin $R$ such that $C=\mathbb{S C o m m i t}(m p k ; R)$, it outputs the random coin $R$ that explains $C$ as a soft commitment.

- EVerify $(m p k, C, R)$ : On input of the public commitment key $m p k$, a commitment $C$ and random coins $R$, it accepts if $R$ is deemed as convincing evidence that $C$ is a soft commitment.

$-R^{\prime} \leftarrow$ FakeExplain $(m s k ; R)$ : On input of the public commitment key $m p k$ and random coins $R$ such that $C=\operatorname{MFake}(m p k ; R)$, this algorithm outputs random coins $R^{\prime}$ such that $C=\mathbb{S C o m m i t}\left(m p k ; R^{\prime}\right)$.

It is straightforward to see that EVerify will only accept if the inputs are soft commitment, explanation or fake commitment, fake explanation pairs. If an adversary can produce explanations for some hard commitment that EVerify accepts, then mercurial binding is broken: The explanation can be adapted to produce soft-openings to any message like fake commitments which contradicts the mercurial binding property that hard commitments can only be hard-/softopened to a unique message.

With these three new algorithms, we require an additional equivocation property, soft-explain (SE) equivocation, for the security of TMC schemes.

- SE Equivocation: The real game provides $\mathcal{A}$ with a soft commitment $C=$ $\mathbb{S C o m m i t}(m p k ; R)$ and the corresponding random coins $R$. The ideal game provides $\mathcal{A}$ with a fake commitment $C=\operatorname{MFake}(m s k ; R)$ and a fake explanation $R^{\prime} \leftarrow$ FakeExplain $(m s k ; R)$ of $C$ as a soft commitment. 
Optimized Proof of Membership for an Interval. A naive method to prove membership of a set of keys of D lying in some interval $[a, b], \mathcal{R}$, is to return $|\mathcal{R}|$ many hard authentication paths. This is sub-optimal as there are duplicated hard openings as authentication paths merge closer to the root of the Merkle tree. We show how the Steiner tree yields the optimal set of hard openings.

For a set of leaves $\mathcal{R} \subseteq[\mathrm{D}] \cap[a, b]$, let $S T(\mathcal{R})$ be the Steiner tree of $\mathcal{R}$, the minimal subtree connecting the leaves of $\mathcal{R}$ to the root. We use $S T(\mathcal{R})_{j}$ to mean the set of nodes in $S T(\mathcal{R})$ at depth $j$. We define the authentication Steiner tree of $\mathcal{R}, \Pi_{\mathcal{R}}$, as the set of hard openings for each node in $S T(\mathcal{R})$ : namely, hard openings $\pi_{x}$ to $\mathrm{D}(x)$ for each leaf $x \in \mathcal{R}$, and $\pi_{y}$ to $\left(C_{y}, C_{y^{\prime}}\right)$ for each internal node $y \in S T(\mathcal{R}) \backslash \mathcal{R}$. By definition, hard authentication paths for all $x \in \mathcal{R}$ are in $\Pi_{\mathcal{R}}$ above which has $\mathcal{O}(|\mathcal{R}| \cdot \ell)$ nodes. The mechanism and its verification is formalized in $\mathbb{H}$ OpenST and VerifyST with $($ com, $\Delta) \leftarrow$ BuildTree $($ crs, D).

- $\Pi_{\mathcal{R}} \leftarrow \mathbb{H}$ OpenST $(\mathrm{crs},(\mathrm{com}, \Delta), \mathcal{R})$ : With inputs CRS crs, database commitment com, decommitment information $\Delta$ and set $\mathcal{R} \subseteq[\mathrm{D}]$, return $\Pi_{\mathcal{R}}$ as follows:

1. For each leaf $x \in \mathcal{R}$, compute $\pi_{x} \leftarrow \mathbb{H}$ Open $\left(\operatorname{crs}, \mathrm{D}(x) ; R_{x}\right)$.

2. For $j$ from $\ell-1$ to 0 and $z \in S T(\mathcal{R})_{j}$, the set of nodes in $S T(\mathcal{R})$ at depth $j$, compute $\pi_{z} \leftarrow \mathbb{H O p e n}\left(\operatorname{crs},\left(C_{z 0}, C_{z 1}\right) ; R_{z}\right)$.

Set $\Pi_{\mathcal{R}}=\left(\left\{(x, \mathrm{D}(x)), \pi_{x}\right\}_{x \in \mathcal{R}},\left\{C_{z}, C_{z^{\prime}}, \pi_{z}\right\}_{z \in S T(\mathcal{R}) \backslash \mathcal{R}}\right)$.

$-\mathcal{L} \leftarrow \operatorname{VerifyST}\left(\right.$ crs, com $\left., \mathcal{R}, \Pi_{\mathcal{R}}\right):$ With inputs CRS crs, database commitment com, set $\mathcal{R}$,

1. For each leaf $x \in \mathcal{R}$, compute $\mathbb{H} V$ erify $\left(\operatorname{crs}, \mathrm{D}(x), C_{x}, \pi_{x}\right)$ and continue if all verifications accept. Otherwise, set and output $\mathcal{L}=$ bad.

2. For $j$ from $\ell-1$ to 0 and $z \in S T(\mathcal{R})_{j}$, the set of nodes in $S T(\mathcal{R})$ at depth $j$, compute $\mathbb{H} \operatorname{Verify}\left(\operatorname{crs},\left(C_{z 0}, C_{z 1}\right), C_{z}, \pi_{z}\right)$ and continue if all verifications accept. Otherwise, set and output $\mathcal{L}=$ bad.

Return $\mathcal{L}=\mathcal{R}$ if $\mathcal{L} \neq$ bad.

Proof of Non-Membership for an Interval. With the authentication Steiner tree for $\mathcal{R}$ proving that keys $x \in \mathcal{R}$ are in [D], we need to show that the other keys in $[a, b] \backslash \mathcal{R}$ do not appear in $\mathrm{D}$. This is achieved with a crucial observation: If an internal node $y^{\prime}$ is a soft commitment or nil, then no descendant of $y^{\prime}$ has a valid hard authentication path and cannot be in [D]. So, we use Explain to prove that no leaves in $[a, b] \backslash \mathcal{R}$ have a hard authentication path to the root. In particular, if $\mathcal{P}$ is the canonical covering of $[a, b] \backslash \mathcal{R}$, we have $C_{x}=$ nil or $\mathbb{S C o m m i t}\left(m p k ; R_{x}\right)$ for any node $x \in \mathcal{P}$ after BuildTree $(c r s, \mathrm{D})$.

The values $C_{z}$ of $z \in \mathcal{P}$ are explained as soft commitments to show that the leaves $[a, b] \backslash \mathcal{R}$ cannnot be involved in a proof of membership. With $\mathcal{R}=$ $\left\{x_{1}, \ldots, x_{m}\right\}$. the canonical coverings of intervals $\left[a, x_{1}-1\right]$ and $\left[x_{m}+1, b\right]$ may not be siblings of nodes in $S T(\mathcal{R})$ but descendants instead. In those cases, we compute soft authentication paths from these canonical coverings to the ancestors which are siblings of some nodes in $S T(\mathcal{R})$. Those $z \in \mathcal{P}$ whose siblings $z^{\prime}$ are in $S T(\mathcal{R})$ do not need additional proof elements. The entire process 
adds $\mathcal{O}(|\mathcal{R}| \cdot \log ((b-a) /|\mathcal{R}|))$ nodes which is only a constant factor larger than $|S T(\mathcal{R})|=|\mathcal{R}| \cdot \ell$. Thus, the entire range proof has size $\mathcal{O}(|\mathcal{R}| \cdot \ell)$, independent of the length of the input interval and allows for exponentially large inputs.

$-\Pi_{[a, b]} \leftarrow \operatorname{Openl}(\mathrm{crs},(\mathrm{com}, \Delta),[a, b])$ : If $a=b$, prove membership of $a$ with $\mathbb{H O p e n P a t h}$ and non-membership with $\mathbf{S O p e n P a t h}$. Otherwise proceed as follows. Let $\mathcal{R}$ be the set of keys in $[\mathrm{D}] \cap[a, b]$.

- If $\mathcal{R}=\emptyset$, set $\Pi_{[a, b], \mathcal{R}}=$ nil and let $\mathcal{P}$ be the canonical covering of the leaves in $[a, b]$. For each $x \in \mathcal{P}$ :

1. Compute $C_{q} \leftarrow \mathbb{S}$ Commit $\left(\right.$ crs $\left.; R_{q}\right)$ if $C_{q}=$ nil for $q=x, x^{\prime}$.

2. Compute $R_{x} \leftarrow \operatorname{Explain}\left(\right.$ crs,$\left.C_{x} ; R_{x}\right)$.

3. For $i$ from $|x|-1$ to 0 , compute $C_{q} \leftarrow \mathbb{S}$ Commit $\left(\right.$ crs $\left.; R_{\left.x\right|_{i}}\right)$ if either of $q=\left.x\right|_{i},\left(\left.x\right|_{i}\right)^{\prime}$ has not been computed previously. Then, compute $\tau_{\left.x\right|_{i}} \leftarrow$ SOpen $\left(\right.$ crs,$\left.\left(C_{\left.x\right|_{i} 0}, C_{\left.x\right|_{i} 1}\right) ; R_{\left.x\right|_{i}}\right)$.

Set $\Pi_{[a, b], \mathcal{P}}=\left\{R_{x},\left\{C_{\left.x\right|_{i} 0}, C_{\left.x\right|_{i} 1}, \tau_{\left.x\right|_{i}}\right\}_{0 \leq i \leq|x|-1}\right\}_{x \in \mathcal{P}}$, proofs that $C_{x}$ is a soft commitment and committed to com for $x \in \mathcal{P}$.

- Otherwise, $\mathcal{R} \neq \emptyset$ and compute the authentication Steiner tree of $\mathcal{R}$, $\Pi_{[a, b], \mathcal{R}} \leftarrow \mathbb{H}$ OpenST $($ crs $,($ com,$\Delta), \mathcal{R})$.

1. (Explain canonical covering.) Let $\mathcal{P}$ be the canonical covering of the keys in $[a, b] \backslash \mathcal{R}$, for $x \in \mathcal{P}$, compute $R_{x} \leftarrow \operatorname{Explain}\left(\operatorname{crs}, C_{x} ; R_{x}\right)$

2. (Prove connection to $S T(\mathcal{R})$.) If $x^{\prime} \notin S T(\mathcal{R})$, let $h_{x}$ be such that $\left.x\right|_{h_{x}}=y^{\prime}$ for some $y \in S T(\mathcal{R})$. For $i$ from $|x|-1$ to $h_{x}$, compute $\tau_{\left.x\right|_{i}} \leftarrow \operatorname{SOpen}\left(\operatorname{crs},\left(C_{\left.x\right|_{i} 0}, C_{\left.x\right|_{i} 1}\right) ; R_{\left.x\right|_{i}}\right)$.

Set $\Pi_{[a, b], \mathcal{P}}=\left\{R_{x},\left\{C_{\left.x\right|_{i} 0}, C_{\left.x\right|_{i} 1}, \tau_{\left.x\right|_{i}}\right\}_{h_{x} \leq i \leq|x|-1}\right\}_{x \in \mathcal{P}}$, proving $C_{x}$ are soft commitments and their paths to com meet $S T(\mathcal{R})$ for $x \in \mathcal{P}_{[a, b]}$.

Output $\Pi_{[a, b]}=\left(\Pi_{[a, b], \mathcal{P}}, \Pi_{[a, b], \mathcal{R}}\right)$ and add the randomness of any commitments computed to $\Delta$.

$-\mathcal{L} \leftarrow \operatorname{Verify}\left(\right.$ crs, com, $\left.\mathfrak{R}, \Pi_{[a, b]}\right):$ If $a=b, \Pi_{[a, b]}=\Pi_{a}$ and compute $y \leftarrow$ VerifyPath (crs, com, $\left.a, \Pi_{a}\right)$. Set $\mathcal{L}^{\prime}=\{(a, y)\}$ if $y \notin\{\perp, b a d\}$ and $\mathcal{L}^{\prime}=\emptyset$ if $y=\perp$. If $a \neq b$, let the proof $\Pi_{[a, b]}=\left(\Pi_{[a, b], \mathcal{P}}, \Pi_{[a, b], \mathcal{R}}\right)$, where $\mathcal{R}$ denotes the set of keys returned. Set $\mathcal{L}^{\prime}=\emptyset$ and proceed as follows.

- If $\mathcal{R}=\emptyset, \Pi_{[a, b], \mathcal{R}}=n i l, \Pi_{[a, b], \mathcal{P}}=\left\{R_{x},\left\{C_{\left.x\right|_{i} 0}, C_{\left.x\right|_{i} 1}, \tau_{\left.x\right|_{i}}\right\}_{0 \leq i \leq|x|-1}\right\}_{x \in \mathcal{P}}$.

For each $x \in \mathcal{P}$, where $\mathcal{P}$ is the canonical covering of $[a, b]$ :

a. Compute EVerify $\left(\mathrm{crs}, C_{x}, R_{x}\right)$ to check that $C_{x}$ is a soft commitment. Set $y=b a d$ if it is not.

b. For $i$ from $|x|-1$ to 0 , compute $\mathbb{S V e r i f y}\left(\operatorname{crs},\left(C_{x||_{0} 0}, C_{x||_{i} 1}\right), C_{x}, \tau_{x}\right)$ and set $y=b a d$ if any verification fails.

- Otherwise, $\left(\Pi_{[a, b], \mathcal{R}}=\left(\left\{(x, \mathrm{D}(x)), \pi_{x}\right\}_{x \in \mathcal{R}},\left\{C_{z}, C_{z^{\prime}}, \pi_{z}\right\}_{z \in S T(\mathcal{R}) \backslash \mathcal{R}}\right)\right.$ and $\Pi_{[a, b], \mathcal{P}}=\left\{R_{x},\left\{C_{\left.x\right|_{i} 0}, C_{\left.x\right|_{i} 1}, \tau_{\left.x\right|_{i}}\right\}_{h_{x} \leq i \leq|x|-1}\right\}_{x \in \mathcal{P}}$.

1. Compute $\mathcal{L}^{\prime} \leftarrow$ VerifyST $\left(\right.$ crs, com, $\left.\mathcal{R}, \Pi_{[a . b], \mathcal{R}}\right)$ to check the authentication Steiner tree.

2. (Check canonical covering of $[a, b] \backslash \mathcal{R}$.) Let $\mathcal{P}$ be the canonical covering of the keys in $[a, b] \backslash \mathcal{R}$. For each $x \in \mathcal{P}$ :

a. Compute EVerify $\left(\operatorname{crs}, C_{x}, R_{x}\right)$ and set $y=$ bad if it fails.

b. For $i$ from $|x|-1$ to $h_{x}$, compute SVerify $\left(\operatorname{crs},\left(C_{x 0}, C_{x 1}\right), \tau_{x}\right)$ and set $y=b a d$ if any verification fails.

If $\mathcal{L}^{\prime}$ and $y \neq b a d$, set and output $\mathcal{L}=\mathcal{L}^{\prime}$. 


\subsection{Range Queries over Records in ZK-EEDB}

Let $(c r s, t k) \leftarrow \operatorname{Init}\left(1^{\lambda}\right)$ and $(c o m, \Delta) \leftarrow \operatorname{ComDB}(c r s, \mathrm{D})$ be the ZK-EEDB commitment and decommitment information of a database $\mathrm{D}$ and consider an arbitrary range $\mathfrak{R}=\left[a_{x}, b_{x}\right] \times\left[a_{y}, b_{y}\right]$. Correctness of its answer is proved in zeroknowledge with several membership and range proofs in the Merkle trees built in ComDB. Due to space constraints, we sketch the range proof generation and verification algorithms, ProveRQ and VerifyRQ and defer its formal description to Appendix B.

ProveRQ. The value Merkle tree can be seen as a two-tiered size-hiding commitment to $\mathrm{D}$, by storing commitments to $\mathrm{D}^{-1}(y)$ for every possible value in the universe $y \in\left[0,2^{\ell}\right)$. Proofs of membership of a record $(x, y)$ on the value Merkle tree would comprise of two parts. First, the committer proves that the value at some leaf $x$ is a hard commitment to 1 in the commitment $\operatorname{com}_{\mathrm{D}_{y}^{-1}}$. This shows that the record $(x, y)$ is in some database committed in some commitment $\operatorname{com}_{\mathrm{D}_{y}^{-1}}$, which we next prove to be the commitment to $\mathrm{D}_{y}^{-1}$. We achieve this by proving that $\operatorname{com}_{\mathrm{D}_{y}^{-1}}$ is committed in the value at leaf $y$ in the ZK-EEDB commitment of the value Merkle tree, $\operatorname{com}_{\mathrm{D}^{-1}}$.

Moving into the sketch of the algorithm, we begin with the most straightforward case: range queries over keys with $\mathfrak{R}=\left[a_{x}, b_{x}\right] \times\left[0,2^{\ell}\right)$. For this, we simply use Openl to prove (non-)membership of all keys in $\left[a_{x}, b_{x}\right]$ of the key Merkle tree. Then, for consistency, we prove that each record $(x, \mathrm{D}(x))$ is committed in the value Merkle tree as well. Next, for range queries over values with range $\left[0,2^{\ell}\right) \times\left[a_{y}, b_{y}\right]$, the procedure is similar. We use Openl on the first tier of the value Merkle tree with the interval $\left[a_{y}, b_{y}\right]$, which proves that some values do not occur in $\mathrm{D}$ and the remaining values store commitments to some non-empty $\mathrm{D}_{y}^{-1}$. After that, we simply reveal the entire Merkle tree for each non-empty $\mathrm{D}_{y}^{-1}$ with Openl on the interval $\left[0,2^{\ell}\right)$. Finally, for consistency, we generate the hard authentication path from leaf $x$ to the root of the key Merkle tree for each record $(x, y)$ that is shown to be in $\mathrm{D}$ from the value Merkle tree.

Finally, we describe the proof generation for range queries over records with $\mathfrak{R}=\left[a_{x}, b_{y}\right] \times\left[a_{y}, b_{y}\right]$. We start in the first tier of the value Merkle tree, and prove that $\operatorname{com}_{\mathrm{D}_{y}^{-1}}$ is the commitment to $\mathrm{D}_{y}^{-1}$ for each $y \in\left[a_{y}, b_{y}\right]$; a hard (resp. soft) authentication path from $y$ to the root of the value Merkle tree is generated for those that are non-empty (resp. empty) in $\left[a_{x}, b_{x}\right]$. Then, for each $y \in \mathrm{D}_{y}^{-1}$, we use Openl to prove (non-)membership of all the keys in the interval $\left[a_{x}, b_{x}\right]$. Consistency is proven in the same way as range queries over values.

VerifyRQ. To verify range proofs, we verify proofs for the key and value Merkle tree separately for the set of records $\mathcal{L}$ returned. For the key Merkle tree, the process is straightforward; we either verify a (non-)membership proof for an interval $\left[a_{x}, b_{x}\right]$ in range queries over keys or a set of hard authentication paths in the other two range queries. Proofs for the value Merkle tree consists of verifying that records are committed in some commitments which purport to be 
of $\mathrm{D}_{y}^{-1}$. These supposed commitments of $\mathrm{D}_{y}^{-1}$ are then verified to be what they are by checking that they are committed in $\operatorname{com}_{\mathrm{D}^{-1}}$. Proofs fail verification if any of the sub-proofs are incorrect.

For range queries over keys, honestly computed range proofs $\Pi$ contain a (non-)membership proof for all keys $x \in\left[a_{x}, b_{x}\right]$ which we verify with Verifyl. Then, for consistency, $\Pi$ also contains individual hard authentication paths for each record $(x, \mathrm{D}(x)) \in \mathcal{L}$ from leaf $x$ to a claimed commitment of $\mathrm{D}^{-1}(\mathrm{D}(x))$ and hard authentication path from leaf $\mathrm{D}(x)$ to the root of the value Merkle tree whose value is $\operatorname{com}_{\mathrm{D}^{-1}}$. These are verified with the authentication path verification algorithm VerifyPath

Let $\mathcal{L}$ denote the set of records returned by the prover and $\Pi$ be the range proof. For range queries over values, the set $\mathcal{L}$ can be partitioned into $\mathcal{L}=$ $\bigcup_{y \in \mathcal{V}}\left\{\left(x_{i}, \mathrm{D}\left(x_{i}\right)=y\right)\right\}$, where $\mathcal{V}$ is the set of unique values that occur in $\mathcal{L}$. Then, we only need to verify (non-)membership proofs for all keys in $\mathbf{D}^{-1}(y)$ for $y \in \mathcal{V}$, to check that the records returned are correctly commited in some claimed commitment of $\mathrm{D}^{-1}(y)$. Finally, we verify (non-)membership proofs for the interval $y \in\left[a_{y}, b_{y}\right]$ of the value Merkle tree using Verifyl to check that the claimed commitments to $\mathrm{D}_{y}^{-1}$ for $y \in\left[a_{y}, b_{y}\right]$ are valid and the remaining $\mathrm{D}_{y}^{-1}$ 's are empty. Consistency checks are straightforward, each record returned is checked to have a valid hard authentication path in $\Pi$ with VerifyPath.

Lastly, for range queries over records, consistency checks are identical to range queries over values and so we focus on the differences in the value Merkle tree. Instead of checking only $y \in \mathcal{V}$, we have to do verify the (non-)membership proof for the interval $\left[a_{x}, b_{x}\right]$ with the claimed $\operatorname{com}_{\mathrm{D}_{y}^{-1}}$ for every $y \in\left[a_{y}, b_{y}\right]$. This is done with Verifyl. Finally, we check that the claimed commitments to $\mathrm{D}_{y}^{-1}$ are correctly committed with valid hard or soft authentication paths, for every $y \in\left[a_{y}, b_{y}\right]$, to the root of the value Merkle tree whose value is $\operatorname{com}_{\mathrm{D}^{-1}}$.

Proof Sizes. There are three cases with different input ranges $\mathfrak{R}$ and proof sizes, which is taken to be the number of nodes to open or explain. Let $\mathcal{L}$ denote the answer to the range query with input $\mathfrak{R}=\left[a_{x}, b_{x}\right] \times\left[a_{y}, b_{y}\right]$.

First, the general case where $\left[a_{x}, b_{x}\right]$ and $\left[a_{y}, b_{y}\right]$ are not $\left[0,2^{\ell}\right)$. We partition $\mathcal{L}=\bigcup_{y \in\left[a_{y}, b_{y}\right]} \mathcal{L}_{y}$ based on the value of the record. The proof consists of $\left(b_{y}-a_{y}\right)$ authentication paths in the value Merkle tree, the same number of authentication Steiner trees, one in every Merkle tree with root value $\operatorname{com}_{\mathrm{D}_{y}^{-1}}$ for $y \in\left[a_{y}, b_{y}\right]$ and finally $|\mathcal{L}|$ authentication paths in the key Merkle tree. The Steiner trees and paths would have $\mathcal{O}\left(\left|\mathcal{L}_{y}\right| \ell\right)$ and $\ell$ nodes each respectively. This brings the total proof size to $\mathcal{O}\left(\left(\left(b_{y}-a_{y}\right)(1+K)+|\mathcal{L}|\right) \ell\right)$ nodes, where $K=\max _{y \in\left[a_{y}, b_{y}\right]}\left|\mathcal{L}_{y}\right|$.

Next, we consider range queries over values with $\mathfrak{R}=\left[0,2^{\ell}\right) \times\left[a_{y}, b_{y}\right]$. Let $\mathcal{V}$ be the set of distinct values in the answer set $\mathcal{L}$, which we partition into disjoint subsets based on the value of the record, i.e., $\mathcal{L}=\bigcup_{y \in \mathcal{V}} \mathcal{L}_{y}$. Since the only difference between this and the general case is that use Openl, we have only one authentication Steiner tree for the value Merkle tree and $|\mathcal{V}|$ many Steiner trees for each $\mathrm{D}_{y}^{-1}$ with $y \in \mathcal{V}$. Therefore, the proof size for this case is $\mathcal{O}((|\mathcal{L}|+|\mathcal{V}|(1+K)) \ell)$ with $K=\max _{y \in \mathcal{V}}\left|\mathcal{L}_{y}\right|$. 
Lastly, for range queries over keys with $\mathfrak{R}=\left[a_{x}, b_{x}\right] \times\left[0,2^{\ell}\right)$, the proof consists of the authentication Steiner tree of $\mathcal{L}$ in the key Merkle tree and $\mathcal{O}(|\mathcal{L}|)$ authentication paths in the Merkle tree and some set of Merkle trees with root value $\operatorname{com}_{\mathrm{D}_{y}^{-1}}$ where $(x, y) \in \mathcal{L}$. In total, the proof size is $\mathcal{O}(|\mathcal{L}| \ell)$.

Overall, ProveRQ supports super-polynomial intervals $\left[a_{x}, b_{x}\right]$ over the keyspace for any query and value-space for range queries over values. For range queries over records, only polynomial length intervals $\left[a_{y}, b_{y}\right]$ are supported.

\subsection{Security of the ZK-EEDB Construction}

Recall that ZK-EEDB has three properties, correctness, soundness and zeroknowledge. Correctness can be verified from the construction of ZK-EEDB easily.

Theorem 1. The ZK-EEDB resulting from this construction is sound if the TMC scheme is mercurial-binding.

Proof. Suppose that the adversary can produce two contradicting range queries with valid proofs, $\mathfrak{R}, \Pi$ and $\mathfrak{R}^{\prime}, \Pi^{\prime}$. There must exist a record $(x, y) \in \mathfrak{R} \cap \mathfrak{R}^{\prime}$ in $\mathcal{L}=\operatorname{VerifyRQ}($ crs, com, $\mathfrak{R}, \Pi)$ but not $\mathcal{L}^{\prime}=\operatorname{VerifyRQ}\left(\right.$ crs, com, $\left.\mathfrak{R}^{\prime}, \Pi^{\prime}\right)$. There are two cases in this situation: (i) There exists another record $\left(x, y^{\prime}\right) \in \mathcal{L}^{\prime}$ such that $y^{\prime} \neq y$; (ii) There exists no $y^{\prime} \in\left[0,2^{\ell}\right)$ such that $\left(x, y^{\prime}\right) \in \mathcal{L}^{\prime}$.

In case (i), both $(x, y)$ and $\left(x, y^{\prime}\right)$ have valid proofs that they are committed in $\operatorname{com}_{\mathrm{D}}$. This means that $\Pi$ and $\Pi^{\prime}$ contain two valid hard decommitments to distinct values in two distinct authentication paths for the leaf $x$ of the Merkle tree of $\operatorname{com}_{\mathrm{D}}$. This breaks the mecurial-binding property of the TMC scheme in the same way as in the proof of the soundness property in ordinary ZK-EDBs.

In case (ii), there exists no record with key $x$ in $\mathcal{L}^{\prime}$. This implies that $\Pi^{\prime}$ contains a proof that $(x, 1) \notin \mathrm{D}_{y}^{-1}$ and therefore $x \notin \mathrm{D}^{-1}(y)$. However, $\Pi$ does contain a proof that $(x, 1) \in \mathrm{D}_{y}^{-1}$, leading to a contradiction between $\Pi$ and $\Pi^{\prime}$.

For this to happen, the first possibility is that the two proofs differ in their commitments $\operatorname{com}_{\mathrm{D}_{y}^{-1}}$ of $\mathrm{D}^{-1}(y)$. If so, the value at leaf $y$ of the Merkle tree of $\operatorname{com}_{\mathrm{D}^{-1}}$ has a valid hard opening to one value in $\Pi$ while in $\Pi^{\prime}$, the value at the leaf or some node along its path to the root is either explained as a soft commitment or soft-opened to a message contradicting the hard opening. This contradicts the mercurial-binding property of the TMC scheme, which says that a mercurial commitment cannot be soft-opened to one message and hardopened to a different one. The second possibility is that the commitments $\operatorname{com}_{\mathrm{D}_{y}^{-1}}$ are identical in both $\Pi$ and $\Pi^{\prime}$ but the two proofs depart within the Merkle tree with root value $\operatorname{com}_{\mathrm{D}_{y}^{-1}}$. Since $\Pi$ proves that $(x, 1) \in \mathrm{D}_{y}^{-1}$, it contains a hard authentication path from leaf $x$ to the root. However, $\Pi^{\prime}$ proves that $(x, 1) \notin \mathrm{D}_{y}^{-1}$, meaning either: (a) The value at leaf $x$ is a soft commitment; (b) Some node along the path from leaf $x$ to the root is explained as a soft commitment in $\Pi^{\prime}$. Either way, $\Pi$ shows that the value at some node is a hard commitment whereas $\Pi^{\prime}$ shows that otherwise, the value at the same node either explained as a soft commitment or soft-opened to a message that contradicts the 
hard opening in $\Pi$. As before, this contradicts the mercurial-binding property of the TMC scheme.

Theorem 2. The ZK-EEDB resulting from this construction satisfies the zeroknowledge property if the TMC scheme satisfies the four equivocation properties.

Proof. The zero-knowledge property follows from the equivocation properties enabled by the trapdoor in the TMC scheme. The ZK-EEDB simulator (SInit, SCom, SProveRQ ${ }^{\mathrm{D}}$ ), which is constructed below, is similar to the ZK-EDB simulator. However, a key change is that $S$ Prove $R Q^{D}$ additionally uses FakeExplain. To simulate range proofs, FakeExplain allows explaining fake commitments as soft commitments when some subtrees have to be proved empty.

$-\left(c r s^{\prime}, s t_{0}\right) \leftarrow \operatorname{SInit}\left(1^{\lambda}\right): \operatorname{Run}(c r s, t k) \leftarrow \operatorname{Init}\left(1^{\lambda}\right)$ and output the common reference string and simulator state $\left(c r s^{\prime}=c r s, s t_{0}=t k\right)$.

- $\left(\operatorname{com}^{\prime}, s t_{1}\right) \leftarrow \mathrm{SCom}\left(s t_{0}\right)$ : Compute $\operatorname{com}_{\mathrm{D}}^{\prime} \leftarrow \operatorname{MFake}\left(c r s^{\prime} ; R_{0}\right)$ and $\operatorname{com}_{\mathrm{D}^{-1}}^{\prime} \leftarrow$ MFake $\left(c r s^{\prime} ; R_{1}\right)$ and output $\left(\operatorname{com}^{\prime}=\left(\operatorname{com}_{\mathrm{D}}^{\prime}, \operatorname{com}_{\mathrm{D}^{-1}}^{\prime}\right), s t_{1}=(R, t k)\right)$.

- $\Pi^{\prime} \leftarrow \operatorname{SProveRQ}^{\mathrm{D}}\left(\mathrm{crs}^{\prime}, s t_{1}, \mathfrak{R}=\left[a_{x}, b_{x}\right] \times\left[a_{y}, b_{y}\right]\right)$ : Obtain the set $\mathcal{S}=\mathrm{D} \cap \mathfrak{R}$ by querying the database oracle and let $\mathcal{S}^{\prime} \in s t_{1}$ contain the commitments and proofs that were computed in previous queries. We denote with $\mathcal{S}_{\mathrm{D}^{-1}}$, the set of distinct values in $\mathcal{S}$. Then, let $[\mathcal{S}]_{\mathrm{D}_{y}^{-1}}$ be the set of keys in $[\mathcal{S}]$ whose values in $\mathrm{D}$ are $y$. Compute $\Pi^{\prime}$ as follows:

1. The answer defines several Steiner trees and paths that are needed to prove the correctness of the answer to the adversary.

a. If $\left[a_{y}, b_{y}\right]=\left[0,2^{\ell}\right)$, then $\mathcal{S}$ induces an authentication Steiner tree, $S T([\mathcal{S}])$ in the Merkle tree of $\operatorname{com}_{\mathrm{D}}^{\prime}$ and $|\mathcal{S}|$ many authentication paths, $\mathcal{L}$ and $\mathcal{L}_{y}$, in the Merkle trees of $\operatorname{com}_{\mathrm{D}^{-1}}^{\prime}$ and $\operatorname{com}_{\mathrm{D}_{y}^{-1}}^{\prime}$ for $y \in \mathcal{S}_{\mathrm{D}^{-1}}$ respectively.

b. If $\left[a_{x}, b_{x}\right]=\left[0,2^{\ell}\right)$, then $\mathcal{S}_{\mathrm{D}^{-1}}$ defines an authentication Steiner tree, $S T\left(\mathcal{S}_{\mathrm{D}^{-1}}\right)$ in the Merkle tree of $\operatorname{com}_{\mathrm{D}^{-1}}^{\prime}$ and similar Steiner trees $S T\left([\mathcal{S}]_{y}\right)$ in the Merkle trees of $\operatorname{com}_{\mathrm{D}_{y}^{-1}}^{\prime}$ for $y \in \mathcal{S}_{\mathrm{D}^{-1}}$. Finally, $[\mathcal{S}]$ defines $|[\mathcal{S}]|$ many authentication paths $\mathcal{L}$ in the Merkle tree of $\operatorname{com}_{\mathrm{D}}^{\prime}$. c. If neither $\left[a_{x}, b_{x}\right]$ not $\left[a_{y}, b_{y}\right]$ are $\left[0,2^{\ell}\right)$, then $\mathcal{S}_{\mathrm{D}^{-1}}$ defines $\left|S T\left(\mathcal{S}_{\mathrm{D}^{-1}}\right)\right|$ paths, $\mathcal{L}$ in the Merkle tree of $\operatorname{com}_{\mathrm{D}^{-1}}^{\prime}$ and Steiner trees $S T\left([\mathcal{S}]_{y}\right)$ in the Merkle trees of $\operatorname{com}_{\mathrm{D}_{y}^{-1}}^{\prime}$ for $y \in \mathcal{S}_{\mathrm{D}^{-1}} .[\mathcal{S}]$ also defines an authentication Steiner tree $S T([\mathcal{S}])$ in the Merkle tree of $\operatorname{com}_{\mathrm{D}}^{\prime}$.

2. For each range type, let $\mathcal{N}$ be the set of nodes in the trees and paths induced by the answer $\mathrm{D} \cap \Re$. Then, for every node $x \in \mathcal{N} \backslash \mathcal{S}^{\prime}$, compute fake commitments $C_{x} \leftarrow \operatorname{MFake}\left(\right.$ crs $\left.; R_{x}\right)$.

3. For the fake commitments created in Step 2 and their parents, compute appropriate hard and soft decommitments and explanations using HHEquivocate, SEquivocate and" FakeExplain to simulate an honest proof.

4. Add the fake commitments, hard and soft decommitments and explanations computed in Steps 1 and 2 to the state $s t_{1}$. 
The output of the simulator is indistinguishable from that of an honest prover because of the equivocation properties of the TMC scheme used. There are two types of outputs from the simulator, the CRS from initialization and fake commitments, decommitments and explanations in proofs to queries from the adversary. The simulated CRS is indistinguishable from a real CRS one as both are trapdoor mercurial commitment keys. From the four equivocation properties of the TMC scheme, the joint distribution of fake commitments and their hard/soft equivocations or explanations are statistically indistinguishable from hard/soft commitments and their hard/soft openings or explanations.

\subsection{More Expressive Queries from Range Queries}

We show that queries over keys, values and records with more interesting statements, such as $k$-nearest neighbours and $k$-minimum/maximum queries can be done with ZK-EEDB. On top of that, any query for D can be restricted to its sub-databases $\left\{\mathrm{D}^{-1}(y)\right\}_{y \in\left[0,2^{\ell}\right)}$. These queries and proof techniques are briefly sketched with details deferred to Appendix Clue to space contraints.

Nearest neighbour queries over keys were previously considered by Ghosh et al. [12] for the three-party setting. Roughly speaking, the query seeks the nearest key $x^{\prime} \in \mathrm{D}$ from an input $x$. Their main observation was that $x$ and $x^{\prime}$ induce an interval $\mathcal{I}$ such that $[\mathrm{D}] \cap \mathcal{I}=\left\{x, x^{\prime}\right\}$. For example, if $x^{\prime}>x$, then $[\mathrm{D}] \cap\left[2 x-x^{\prime}, x^{\prime}\right]$ would only contain $x$ and $x^{\prime}$. We note that this principle extends to $k$ neighbours, where the $k$ nearest keys to $x,\left\{x_{1}, \ldots, x_{k}\right\}$ is returned instead. With ZK-EEDB, we can even extend these queries to values and records.

Related to the $k$-nearest neighbours query are the minimum and maximum queries. For these, the input is a range $\mathfrak{R}=\left[a_{x}, b_{x}\right] \times\left[a_{y}, b_{y}\right]$ and the answer should be the record $(x, y) \in \mathrm{D}$ such that $(x, y)$ is the minimum or maximum among all records in $\mathrm{D} \cap \mathfrak{R}$. In ZK-EEDB, these are proved by showing that there are no records between $(x, y)$ and either $\left(a_{x}, a_{y}\right)$ or $\left(a_{y}, b_{y}\right)$ depending on if it was a minimum or maximum query. Just like the nearest neighbour query above, these queries can be extended to return the $k$ smallest or largest ( $k$-minimum or $k$-maximum) records and support queries over values and records.

Finally, we highlight an important feature of ZK-EEDB that is not possible with ZK-EDB. In ZK-EEDB, any query, be it range, $k$-nearest neighbours, $k$ minimum or $k$-maximum, can be specialized to $\mathrm{D}^{-1}(y)$. This allows us restrict the query to only records in $\mathrm{D}$ with a particular value $y$. ZK-EDB cannot handle

this restriction as we would have to prove that the value at each leaf not returned is either "not $y$ " or "is $y$ but does not satisfy the query".

\section{Lattice Instantiations}

\subsection{A Trapdoor Mercurial Commitment from Standard Lattices}

Let $\lambda \in \mathbb{N}$ be a security parameter. The scheme works with message space $\mathcal{M}=\{0,1\}^{l}$, where $l \in$ poly $(\lambda)$. For a dimension $n=\mathcal{O}(\lambda)$ and prime modulus 
$q=\widetilde{\mathcal{O}}\left(l \cdot n^{2}+n^{4}\right)$, let $w=n\lceil\log q\rceil, \bar{m}=2 n\lceil\log q\rceil$ and $m=\bar{m}+w$. Choose a Gaussian parameter $\sigma=\Omega(\sqrt{n \log q \log n})$.

$-(m p k, m s k) \leftarrow \operatorname{Setup}\left(1^{\lambda}\right):$ Choose a matrix $\mathbf{A}_{0} \hookleftarrow U\left(\mathbb{Z}_{q}^{n \times l}\right)$. Run algorithm $\operatorname{TrapGen}(n, m, q)$ (Lemma 4 ) to generate a pair $\left(\mathbf{A}_{1}, \mathbf{T}\right)$, where $\mathbf{A}_{1} \in \mathbb{Z}_{q}^{n \times m}$ is statistically close to uniform and $\mathbf{T} \in \mathbb{Z}^{\bar{m} \times w}$ is its trapdoor.

Output $m p k=\left(\mathbf{A}_{0}, \mathbf{A}_{1}\right)$ and $m s k=\mathbf{T}$.

- $\mathbf{C} \leftarrow \mathbb{H}$ Commit $(m p k, \boldsymbol{\mu} ;(\mathbf{R}, \mathbf{r}))$ : Given a message $\boldsymbol{\mu} \in\{0,1\}^{l}$ and randomness $\mathbf{R} \hookleftarrow D_{\mathbb{Z}^{m \times w}, \sigma}$ and $\mathbf{r} \hookleftarrow D_{\mathbb{Z}^{m+w}, \sigma}$, define $\mathbf{B}=\left[\mathbf{A}_{1} \mid \mathbf{B}_{1}\right] \in \mathbb{Z}_{q}^{n \times(m+w)}$, where $\mathbf{B}_{1}=\mathbf{A}_{1} \cdot \mathbf{R} \in \mathbb{Z}_{q}^{n \times w}$. Then, compute $\mathbf{c}=\mathbf{A}_{0} \cdot \boldsymbol{\mu}+\mathbf{B} \cdot \mathbf{r} \in \mathbb{Z}_{q}^{n}$ and output the hard commitment $\mathbf{C}=\left(\mathbf{c}, \mathbf{B}_{1}\right) \in \mathbb{Z}_{q}^{n} \times \mathbb{Z}_{q}^{n \times w}$.

- $\pi \leftarrow \mathbb{H}$ Open $\left(m p k, \mu ;(\mathbf{R}, \mathbf{r})\right.$ : Output $\pi=(\mathbf{R}, \mathbf{r}) \in \mathbb{Z}^{m \times w} \times \mathbb{Z}^{m+w}$.

- $\mathbb{H}$ Verify $(m p k, \boldsymbol{\mu}, \mathbf{C}, \pi)$ : Given a commitment $\mathbf{C}=\left(\mathbf{c}, \mathbf{B}_{1}\right) \in \mathbb{Z}_{q}^{n} \times \mathbb{Z}_{q}^{n \times w}$ and a purported hard opening $\pi=(\mathbf{R}, \mathbf{r})$, proceed as follows.

1. Return 0 if $\mathbf{R}=\left[\mathbf{r}_{1}|\ldots| \mathbf{r}_{w}\right]$ has a column such that $\left\|\mathbf{r}_{i}\right\|>\sigma \sqrt{m}$ or if $\|\mathbf{r}\|>\sigma \sqrt{m+w}$.

2. Let $\mathbf{B}=\left[\mathbf{A}_{1} \mid \mathbf{B}_{1}\right] \in \mathbb{Z}_{q}^{n \times(m+w)}$. Return 1 if $\mathbf{B}_{1}=\mathbf{A}_{1} \cdot \mathbf{R}$ and $\mathbf{c}=$ $\mathbf{A}_{0} \cdot \boldsymbol{\mu}+\mathbf{B} \cdot \mathbf{r}$.

- $\mathbf{C} \leftarrow \mathbb{S}$ Commit $(m p k ;(\mathbf{R}, \mathbf{r}))$ : Given $\mathbf{R} \hookleftarrow D_{\mathbb{Z}^{m \times w}, \sigma}$ and $\mathbf{r} \hookleftarrow D_{\mathbb{Z}^{m+w}, \sigma}$, compute the matrix $\mathbf{B}=\left[\mathbf{A}_{1} \mid \mathbf{G}-\mathbf{A}_{1} \cdot \mathbf{R}\right] \in \mathbb{Z}_{q}^{n \times(m+w)}$ and $\mathbf{c}=\mathbf{B} \cdot \mathbf{r} \in \mathbb{Z}_{q}^{n}$. Output $\mathbf{C}=\left(\mathbf{c}, \mathbf{B}_{1}\right) \in \mathbb{Z}_{q}^{n} \times \mathbb{Z}_{q}^{n \times w}$, where $\mathbf{B}_{1}=\mathbf{G}-\mathbf{A}_{1} \cdot \mathbf{R}$. Note that matrix $\mathbf{R}$ is a trapdoor for $\mathbf{B}$.

$-\tau \leftarrow \operatorname{SOpen}(m p k, \boldsymbol{\mu}$, flag; $(\mathbf{R}, \mathbf{r}))$ :

- If flag $=\mathbb{S}$, we must have $\mathbf{C}=\left(\mathbf{c}, \mathbf{B}_{1}\right)=\left(\mathbf{B} \cdot \mathbf{r}, \mathbf{G}-\mathbf{A}_{1} \cdot \mathbf{R}\right)$. Compute $\mathbf{c}^{\prime}=\mathbf{c}-\mathbf{A}_{0} \cdot \boldsymbol{\mu}$ and sample $\mathbf{r}^{\prime} \leftarrow \operatorname{SampleD}\left(\mathbf{R}, \mathbf{B}, \mathbf{c}^{\prime}, \sigma\right)$ (Lemma 4 ).

Then, output $\tau=\mathbf{r}^{\prime} \in \mathbb{Z}^{m+w}$, which satisfies $\mathbf{c}=\mathbf{A}_{0} \cdot \boldsymbol{\mu}+\mathbf{B} \cdot \mathbf{r}^{\prime}$ and $\left\|\mathbf{r}^{\prime}\right\| \leq \sigma \sqrt{m+w}$ with overwhelming probability (Lemma 1).

- If flag $=\mathbb{H}$, output $\tau=\mathbf{r} \in \mathbb{Z}^{m+w}$.

- $\operatorname{SVerify}(m p k, \boldsymbol{\mu}, \mathbf{C}, \tau)$ : Let $\mathbf{C}=\left(\mathbf{c}, \mathbf{B}_{1}\right) \in \mathbb{Z}_{q}^{n} \times \mathbb{Z}_{q}^{n \times w}$ and $\tau=\mathbf{r} \in \mathbb{Z}^{m+w}$ and define $\mathbf{B}=\left[\mathbf{A}_{1} \mid \mathbf{B}_{1}\right] \in \mathbb{Z}_{q}^{n \times(m+w)}$. Return 1 if $\mathbf{c}=\mathbf{A}_{0} \cdot \boldsymbol{\mu}+\mathbf{B} \cdot \mathbf{r}$ and $\|\mathbf{r}\| \leq \sigma \sqrt{m+w}$. Otherwise, return 0 .

- $\mathbf{C} \leftarrow$ MFake $(m p k ;(\mathbf{R}, \mathbf{r}))$ : Given $\mathbf{R} \hookleftarrow D_{\mathbb{Z}^{m \times w}, \sigma}$ and $\mathbf{r} \hookleftarrow D_{\mathbb{Z}^{m+w}, \sigma}$, compute $\mathbf{B}=\left[\mathbf{A}_{1} \mid \mathbf{B}_{1}\right] \in \mathbb{Z}_{q}^{n \times(m+w)}$, where $\mathbf{B}_{1}=\mathbf{A}_{1} \cdot \mathbf{R}$, and compute $\mathbf{c}=\mathbf{B} \cdot \mathbf{r}$. Output $\mathbf{C}=\left(\mathbf{c}, \mathbf{B}_{1}\right)$.

$-\pi \leftarrow \mathbb{H}$ Equivocate $(m s k, \boldsymbol{\mu} ;(\mathbf{R}, \mathbf{r}))$ : Let $m s k=\mathbf{T} \in \mathbb{Z}^{\bar{m} \times w}$ and let the fake commitment be $\mathbf{C}=\left(\mathbf{c}, \mathbf{B}_{1}\right)=\left(\mathbf{B} \cdot \mathbf{r}, \mathbf{A}_{1} \cdot \mathbf{R}\right)$, where $\mathbf{B}=\left[\mathbf{A}_{1} \mid \mathbf{A}_{1} \cdot \mathbf{R}\right]$. Compute $\mathbf{c}^{\prime}=\mathbf{c}-\mathbf{A}_{0} \cdot \boldsymbol{\mu}$. Then, extend $\mathbf{T}$ into a trapdoor $\mathbf{T}_{\mathbf{B}}$ for the matrix $\mathbf{B}=\left[\mathbf{A}_{1} \mid \mathbf{A}_{1} \cdot \mathbf{R}\right]$ and sample $\mathbf{r}^{\prime} \leftarrow \operatorname{SampleD}\left(\mathbf{T}_{\mathbf{B}}, \mathbf{B}, \mathbf{c}^{\prime}, \sigma\right)$. Output $\pi=\left(\mathbf{R}, \mathbf{r}^{\prime}\right) \in \mathbb{Z}^{m \times w} \times \mathbb{Z}^{m+w}$. 
$-\tau \leftarrow$ SEquivocate $(m s k, \boldsymbol{\mu} ;(\mathbf{R}, \mathbf{r}))$ : Let $m s k=\mathbf{T} \in \mathbb{Z}^{\bar{m} \times w}$ and let the fake commitment be $\mathbf{C}=\left(\mathbf{c}, \mathbf{B}_{1}\right)=\left(\mathbf{B} \cdot \mathbf{r}, \mathbf{A}_{1} \cdot \mathbf{R}\right)$, where $\mathbf{B}=\left[\mathbf{A}_{1} \mid \mathbf{A}_{1} \cdot \mathbf{R}\right]$. Compute $\mathbf{c}^{\prime}=\mathbf{c}-\mathbf{A}_{0} \cdot \boldsymbol{\mu}$. Then, extend $\mathbf{T}$ into a trapdoor $\mathbf{T}_{\mathbf{B}}$ for the matrix $\mathbf{B}=\left[\mathbf{A}_{1} \mid \mathbf{A}_{1} \cdot \mathbf{R}\right]$ and sample $\mathbf{r}^{\prime} \leftarrow \operatorname{SampleD}\left(\mathbf{T}_{\mathbf{B}}, \mathbf{B}, \mathbf{c}^{\prime}, \sigma\right)$. Output $\tau=\mathbf{r}^{\prime} \in \mathbb{Z}^{m+w}$.

- $\left(\mathbf{R}^{\prime}, \mathbf{r}^{\prime}\right) \leftarrow$ FakeExplain $(m s k ;(\mathbf{R}, \mathbf{r}))$ : Given $m s k=\mathbf{T} \in \mathbb{Z}^{\bar{m} \times w}$ together with a Gaussian matrix $\mathbf{R}=\left[\mathbf{r}_{1}|\ldots| \mathbf{r}_{w}\right] \hookleftarrow D_{\mathbb{Z}^{m \times w}, \sigma}$ and a vector $\mathbf{r} \hookleftarrow D_{\mathbb{Z}^{m+w}, \sigma}$ such that $\mathbf{C}=\left(\mathbf{c}, \mathbf{B}_{1}\right)=\left(\mathbf{B} \cdot \mathbf{r}, \mathbf{A}_{1} \cdot \mathbf{R}\right)$ is a fake commitment, set $\mathbf{r}^{\prime}=\mathbf{r}$ and use the trapdoor $\mathbf{T}$ for $\mathbf{A}_{1}$ to sample a small-norm $\mathbf{R}^{\prime}=\left[\mathbf{r}_{1}^{\prime}|\ldots| \mathbf{r}_{w}^{\prime}\right]$ such that $\mathbf{A}_{1} \cdot \mathbf{R}^{\prime}=\mathbf{G}-\mathbf{A}_{1} \cdot \mathbf{R}$. To do this, let $\mathbf{G}=\left[\mathbf{g}_{1}|\ldots| \mathbf{g}_{w}\right]$, and for each $i \in[w]$, sample $\mathbf{r}_{i}^{\prime} \leftarrow \operatorname{SampleD}\left(\mathbf{T}, \mathbf{A}_{1}, \mathbf{g}_{i}-\mathbf{A}_{1} \cdot \mathbf{r}_{i}\right)$.

Then, output $\left(\mathbf{R}^{\prime}, \mathbf{r}^{\prime}\right)$ which satisfy $\mathbf{C}=\left(\mathbf{c}, \mathbf{B}_{1}\right)=\left(\mathbf{B} \cdot \mathbf{r}^{\prime}, \mathbf{G}-\mathbf{A}_{1} \cdot \mathbf{R}^{\prime}\right)$.

\subsection{Analysis}

We prove that the trapdoor mercurial commitment scheme described in Section 5.1 satisfies the correctness and security properties defined in Section 2.1.

Correctness. By Lemma 1, with overwhelming probability, samples from discrete Gaussian distributions $D_{\mathbb{Z}^{m}, \sigma}$ and $D_{\mathbb{Z}^{m+w}, \sigma}$ have their Euclidean norms bounded by $\sigma \sqrt{m}$ and $\sigma \sqrt{m+w}$, respectively. Moreover, the outputs of SampleD are statistically close to discrete Gaussian samples, by Lemma 4 . Therefore, if proofs $\pi$ and $\tau$ are generated as in Section 5.1, then they should pass the verifications for Euclidean norms performed by algorithms $\mathbb{H} V$ Verify and $\mathbb{S} V e r i f y . ~ N o t e$ further that the equations modulo $q$ verified by these algorithms must hold by construction. As a result, the scheme is correct with overwhelming probability.

Security. In the following lemmas, we show that the proposed scheme satisfies mercurial-binding under the SIS assumption, and HH, HS, SS and SE equivocation in the statistical sense.

Lemma 5. The scheme is mercurial-binding under the $\mathrm{SIS}_{n, m, q, \beta}$ assumption, with $\beta=\sigma \cdot\left(l \sqrt{\bar{m}}+\sqrt{\sigma m \bar{m}\left(\sigma^{2} w^{3}+2 m\right)}\right)$.

Proof. Since the scheme is a proper mercurial commitment (i.e., hard openings contain their corresponding soft opening as a proper subset), we only need to consider the hard-soft case. Towards a contradiction, let us assume that the adversary can come up with a commitment $\mathbf{C}=\left(\mathbf{c}, \mathbf{B}_{1}\right) \in \mathbb{Z}_{q}^{n} \times \mathbb{Z}_{q}^{n \times w}$ which it can hard-open to a message $\boldsymbol{\mu}$ and soft-opened to a different message $\boldsymbol{\mu}^{\prime}$. This means that the adversary can output $(\boldsymbol{\mu}, \mathbf{R}, \mathbf{r}) \in\{0,1\}^{l} \times \mathbb{Z}^{m \times w} \times \mathbb{Z}^{m+w}$ and $\left(\boldsymbol{\mu}^{\prime}, \mathbf{r}^{\prime}\right) \in\{0,1\}^{l} \times \mathbb{Z}^{m+w}$ such that $\mathbf{B}_{1}=\mathbf{A}_{1} \cdot \mathbf{R}$ and

$$
\mathbf{c}=\mathbf{A}_{0} \cdot \boldsymbol{\mu}+\left[\mathbf{A}_{1} \mid \mathbf{A}_{1} \cdot \mathbf{R}\right] \cdot \mathbf{r}=\mathbf{A}_{0} \cdot \boldsymbol{\mu}^{\prime}+\left[\mathbf{A}_{1} \mid \mathbf{A}_{1} \cdot \mathbf{R}\right] \cdot \mathbf{r}^{\prime} .
$$

Assuming that such a mercurial-binding adversary $\mathcal{A}$ exists, we can build a $\mathrm{SIS}_{n, m, q, \beta}$ solver $\mathcal{B}$ which takes as input a $\mathrm{SIS}_{n, m, q, \beta}$ instance $\mathbf{A} \in \mathbb{Z}_{q}^{n \times \bar{m}}$ and finds a non-zero vector $\mathbf{v}^{\star} \in \mathbb{Z}^{\bar{m}}$ of $\Lambda^{\perp}(\mathbf{A})$ such that $\left\|\mathbf{v}^{\star}\right\| \leq \beta$. To this end, $\mathcal{B}$ samples $\mathbf{R}_{0} \hookleftarrow D_{\mathbb{Z}, \sigma}^{\bar{m} \times l}, \mathbf{R}_{1} \hookleftarrow D_{\mathbb{Z}, \sigma}^{\bar{m} \times m}$ and defines 


$$
\mathbf{A}_{0}=\mathbf{A} \cdot \mathbf{R}_{0} \in \mathbb{Z}_{q}^{n \times l}, \quad \mathbf{A}_{1}=\mathbf{A} \cdot \mathbf{R}_{1} \in \mathbb{Z}_{q}^{n \times m} .
$$

Note that, by Lemma 2, matrices $\mathbf{A}_{0}$ and $\mathbf{A}_{1}$ are statistically close to the distributions $U\left(\mathbb{Z}_{q}^{n \times l}\right)$ and $U\left(\mathbb{Z}_{q}^{n \times m}\right)$, respectively. The adversary $\mathcal{A}$ is given $m p k=\left(\mathbf{A}_{0}, \mathbf{A}_{1}\right)$ and, assuming that it can output $(\boldsymbol{\mu}, \mathbf{R}, \mathbf{r})$ and $\left(\boldsymbol{\mu}^{\prime}, \mathbf{r}^{\prime}\right)$ satisfying (1) for distinct $\boldsymbol{\mu} \neq \boldsymbol{\mu}^{\prime}$, we have

$$
\left.\mathbf{A}_{0} \cdot\left(\boldsymbol{\mu}-\boldsymbol{\mu}^{\prime}\right)\right)=\mathbf{A}_{1} \cdot\left[\mathbf{I}_{m} \mid \mathbf{R}\right] \cdot\left(\mathbf{r}^{\prime}-\mathbf{r}\right) \quad \bmod q .
$$

This implies that

$$
\mathbf{v}^{\star}=\mathbf{R}_{0} \cdot\left(\boldsymbol{\mu}-\boldsymbol{\mu}^{\prime}\right)+\mathbf{R}_{1} \cdot\left[\mathbf{I}_{m} \mid \mathbf{R}\right] \cdot\left(\mathbf{r}-\mathbf{r}^{\prime}\right) \in \mathbb{Z}^{\bar{m}}
$$

is a short vector of $\Lambda^{\perp}(\mathbf{A})$ with norm $\left\|\mathbf{v}^{\star}\right\| \leq \sigma \cdot\left(l \sqrt{\bar{m}}+\sqrt{\sigma m \bar{m}\left(\sigma^{2} w^{3}+2 m\right)}\right)$. Moreover, we claim that it is non-zero with overwhelming probability. Indeed, $\left(\boldsymbol{\mu}-\boldsymbol{\mu}^{\prime}\right) \in\{-1,0,1\}^{l}$ has at least one non-zero coordinate by hypothesis. Given that the columns of $\mathbf{R}_{0}$ have at least $\Omega(n)$ bits of min-entropy conditionally on $\mathbf{A}_{0}=\mathbf{A} \cdot \mathbf{R}_{0}$ (by Lemma 2 and Lemma 3 , the product $\mathbf{R}_{0} \cdot\left(\boldsymbol{\mu}-\boldsymbol{\mu}^{\prime}\right)$ is a linear combination (with coefficients in $\{-1,0,1\}$ ) of the columns of $\mathbf{R}_{0}$ which contains a completely unpredictable term. Hence, the right-hand-side member of (2) can only cancel over $\mathbb{Z}^{\bar{m}}$ with negligible probability.

Lemma 6. The scheme provides $H H, H S, S S$ and $S E$ equivocation in the statistical sense.

Proof. For any message $\boldsymbol{\mu}$, we show that the distribution of fake commitments and their hard equivocations to $\boldsymbol{\mu}$ is statistically close to that of hard commitments and their hard openings to $\boldsymbol{\mu}$.

We note that $\mathbf{B}_{1}$ is generated in the same way in both fake and hard commitments. Moreover, since $\mathbf{A}_{1}$ is statistically uniform over $\mathbb{Z}_{q}^{n \times m}$, Lemma 2 implies that the distribution $\left\{\left(\mathbf{A}_{1}, \mathbf{B}_{1}\right)=\left(\mathbf{A}_{1}, \mathbf{A}_{1} \cdot \mathbf{R}\right) \mid \mathbf{R} \hookleftarrow D_{\mathbb{Z}^{m \times w}, \sigma}\right\}$ is statistically close to the distribution $U\left(\mathbb{Z}_{q}^{n \times m}\right) \times U\left(\mathbb{Z}_{q}^{n \times w}\right)$, meaning $\mathbf{B} \sim U\left(\mathbb{Z}_{q}^{n \times(m+w)}\right)$ in both hard and fake commitments. By Lemma 2 , we find that the distribution of fake commitments $\left(\mathbf{c}, \mathbf{B}_{1}\right)$, which is given by $\left\{\left(\left[\mathbf{A}_{1} \mid \mathbf{B}_{1}\right] \cdot \mathbf{r}, \mathbf{B}_{1}\right) \mid \mathbf{r} \hookleftarrow D_{\mathbb{Z}^{m+w}, \sigma}\right\}$, is in turn statistically close to $U\left(\mathbb{Z}_{q}^{n}\right) \times U\left(\mathbb{Z}_{q}^{n \times(m+w)}\right)$. This implies that the distribution of fake commitments remains statistically unchanged if we compute $\mathbf{c}$

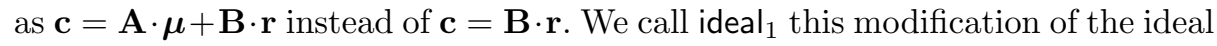
experiment. Moreover, by Lemma 2 again, we know that, for any statistically uniform matrix $\mathbf{A} \sim U\left(\mathbb{Z}_{q}^{n \times(m+w)}\right)$, the distribution

$$
\left\{(\mathbf{A}, \mathbf{A} \cdot \mathbf{r}, \mathbf{r}) \in \mathbb{Z}_{q}^{n \times(m+w)} \times \mathbb{Z}_{q}^{n} \times \mathbb{Z}^{m+w} \mid \mathbf{r} \hookleftarrow D_{\mathbb{Z}^{m+w}, \sigma}\right\}
$$

is statistically close to

$$
\left\{(\mathbf{A}, \mathbf{u}, \mathbf{r}) \in \mathbb{Z}_{q}^{n \times(m+w)} \times \mathbb{Z}_{q}^{n} \times \mathbb{Z}^{m+w} \mid \mathbf{u} \hookleftarrow U\left(\mathbb{Z}_{q}^{n}\right), \mathbf{r} \hookleftarrow D_{\Lambda^{\mathrm{u}}(\mathbf{A}), \sigma}\right\} .
$$

Consequently, we can modify ideal ${ }_{1}$ by changing the way to equivocate the fake commitment. Instead of using extending $\mathbf{T}$ into a trapdoor for $\mathbf{B}=\left[\mathbf{A}_{1} \mid \mathbf{B}_{1}\right]$ 
and using it to sample $\mathbf{r}$ in a coset of the lattice $\Lambda^{\perp}(\mathbf{B})$, we just reveal the vector $\mathbf{r} \hookleftarrow D_{\mathbb{Z}^{m+w}, \sigma}$ that was used to compute $\mathbf{c}=\mathbf{A} \cdot \boldsymbol{\mu}+\mathbf{B} \cdot \mathbf{r}$. If we call this experiment ideal ${ }_{2}$, we find it statistically indistinguishable from the ideal experiment thanks to the statistical closeness of (3)-(4). We observe that ideal 2 is nothing but the real $\mathrm{HH}$ equivocation experiment since $\mathbf{B}_{1}$ is generated in the same way in both experiments. This shows the HH equivocation property. The HS and SS equivocation properties can be shown in a completely similar way.

As for the SE equivocation property, it follows from two observations. First, Lemma 2 implies that the distributions

$$
D_{\text {fake }}:=\left\{\mathbf{A}_{1} \cdot \mathbf{R} \mid \mathbf{R} \hookleftarrow D_{\mathbb{Z}^{m \times w}, \sigma}\right\}, \quad D_{\text {soft }}:=\left\{\mathbf{G}-\mathbf{A}_{1} \cdot \mathbf{R}^{\prime} \mid \mathbf{R}^{\prime} \hookleftarrow D_{\mathbb{Z}^{m \times w}, \sigma}\right\}
$$

are both statistically close to $U\left(\mathbb{Z}_{q}^{n \times w}\right)$. Hence, the adversary's view remains statistically the same if we generate fake commitments by sampling $\mathbf{B}_{1}$ from $D_{\text {soft }}$ instead of $D_{\text {fake }}$ in the ideal experiment. Moreover, since distributions (3) and (4) are statistically close, $\mathcal{A}$ 's view remains statistically the same after modification. instead of using the trapdoor $\mathbf{T}$ of $\Lambda^{\perp}\left(\mathbf{A}_{1}\right)$, we reveal the Gaussian matrix $\mathbf{R}^{\prime}$, used to get $\mathbf{B}_{1}=\mathbf{G}-\mathbf{A}_{1} \cdot \mathbf{R}^{\prime}$ after sampling $\mathbf{R}^{\prime} \hookleftarrow D_{\mathbb{Z}^{m \times w}, \sigma}$. With this, the result is identical to the real game, proving the SE property.

\subsection{Remarks}

The scheme from Section 5.1 produces commitments of the form $\mathbf{C}=\left(\mathbf{c}, \mathbf{B}_{1}\right) \in$ $\mathbb{Z}_{q}^{n} \times \mathbb{Z}_{q}^{n \times w}$, and thus, have length $k=n(w+1)\lceil\log q\rceil$ bits. Its message space is $\mathcal{M}=\{0,1\}^{l}$, where $l$ can vary depending on the context.

The scheme leads to a lattice-based ZK-EEDB system, following the constructions of Sections 3 and 4 . In this system, the following 4 different message lengths, $\left\{l_{1}, l_{2}, l_{3}, l_{4}\right\}$, are considered.

1. At leaves of the first tree, we commit to values of bit-length $l_{1}=\ell$.

2. At non-leaf nodes in both trees, since we commit to 2 commitment strings, we work with message length $l_{2}=2 k$.

3. At leaves of the second tree, we store commitments to $\mathrm{D}^{-1}(y)$, which is a commitment string of bit-length $l_{3}=k$.

4. When building a commitment of $\mathrm{D}_{y}^{-1}=\{(x, 1) \mid(x, y) \in \mathrm{D}\}$, we also work with message length $l_{4}=1$.

To handle these message lengths, we need only adjust the number of columns in $\mathbf{A}_{0} \in \mathbb{Z}_{q}^{n \times l}$, with $l=\max \left\{l_{1}, l_{2}, l_{3}, l_{4}\right\}$. For each $i \in[4]$, we use $\mathbf{A}_{0, i} \in \mathbb{Z}_{q}^{n \times l_{i}}$, the matrix that is the first $l_{i}$ columns of $\mathbf{A}_{0}$, to commit to a length- $l_{i}$ message.

A description of an authentication path with its commitment strings requires $\zeta=\mathcal{O}(l \cdot k)$ bits, which is $\widetilde{\mathcal{O}}\left(\lambda^{3}\right)$ when $l=\mathcal{O}(\lambda)$. Fortunately, this can be greatly reduced if the TMC scheme is adapted to the ring setting. As shown by Micciancio and Peikert [22] and later by Ducas and Micciancio [8], with appropriate choice of parameters, all the lattice-based cryptographic ingredients of Section 2.3 can be adapted to the ring setting. This lets us use $w=\mathcal{O}(\log q)$ (instead of $w=\mathcal{O}(n \log q)$ ), thereby reducing the commitment size and $\zeta$ by a factor of $\mathcal{O}(\lambda)$. Details of the adaptation are available in Appendix $\mathrm{F}$ 
Acknowledgements. Part of this research was funded by Singapore Ministry of Education under Research Grant MOE2016-T2-2-014(S). Another part was funded by BPI-France in the context of the national project RISQ (P141580). This work was also supported in part by the European Union PROMETHEUS project (Horizon 2020 Research and Innovation Program, grant 780701). Khoa Nguyen was also supported by the Gopalakrishnan - NTU Presidential Postdoctoral Fellowship 2018. Huaxiong Wang was also supported by the National Research Foundation, Prime Minister's Office, Singapore under its Strategic Capability Research Centres Funding Initiative.

\section{References}

1. M. Blum, P. Feldman, and S. Micali. Non-interactive zero-knowledge and its applications. In STOC, pages 103-112. ACM, 1988.

2. D. Catalano, Y. Dodis, and I. Visconti. Mercurial commitments: Minimal assumptions and efficient constructions. In TCC 2006, 2006.

3. D. Catalano and D. Fiore. Vector commitments and their applications. In $P K C$, 2013.

4. D. Catalano, D. Fiore, and M. Messina. Zero-knowledge sets with short proofs. In Eurocrypt, 2008.

5. M. Chase, A. Healy, A. Lysyanskaya, T. Malkin, and L. Reyzin. Mercurial commitments with applications to zero-knowledge sets. In EUROCRYPT 2005, 2005.

6. M. Chase, A. Healy, A. Lysyanskaya, T. Malkin, and L. Reyzin. Mercurial commitments with applications to zero-knowledge sets. J. of Cryptology, 2013.

7. M. Chase and I. Visconti. Secure database commitments and universal arguments of quasi knowledge. In Crypto, 2012.

8. L. Ducas and D. Micciancio. Improved short lattice signatures in the standard model. In CRYPTO 2014, 2014.

9. R. Gennaro and S. Micali. Independent zero-knowledge sets. In ICALP, 2006.

10. C. Gentry, C. Peikert, and V. Vaikuntanathan. Trapdoors for Hard Lattices and New Cryptographic Constructions. In STOC 2008, 2008.

11. E. Ghosh, O. Ohrimenko, and R. Tamassia. Verifiable order queries and order statistics on a list in zero-knowledge. In ACNS, 2015.

12. E. Ghosh, O. Ohrimenko, and R. Tamassia. Efficient verifiable range and closest point queries in zero-knowledge. PoPETs, 2016(4), 2016.

13. V. Goyal, R. Ostrovsky, A. Scafuro, and I. Visconti. Black-box non-black-box zero knowledge. In STOC, 2014.

14. Y. Ishai, E. Kushilevitz, R. Ostrovksy, and A. Sahai. Zero-knowledge from secure multiparty computation. In STOC, 2007.

15. A. Kate, G. Zaverucha, and I. Goldberg. Constant-size commitments to polynomials and their applications. In Asiacrypt, volume 6477 of LNCS, pages 177-194. Springer, 2010.

16. A. Kawachi, K. Tanaka, and K. Xagawa. Concurrently secure identification schemes based on the worst-case hardness of lattice problems. In ASIACRYPT 2008, 2008.

17. B. Libert and M. Yung. Concise mercurial vector commitments and independent zero-knowledge sets with short proofs. In TCC 2010, 2010.

18. M. Liskov. Updatable zero-knowledge databases. In Asiacrypt, 2005.

19. V. Lyubashevsky. Fiat-Shamir with Aborts: Applications to Lattice and FactoringBased Signatures. In Asiacrypt, 2009. 
20. R. C. Merkle. A Certified Digital Signature. In CRYPTO, volume 435 of $L N C S$, pages 218-238. Springer, 1989.

21. S. Micali, M. O. Rabin, and J. Kilian. Zero-knowledge sets. In 44th FOCS, 2003.

22. D. Micciancio and C. Peikert. Trapdoors for lattices: Simpler, tighter, faster, smaller. In EUROCRYPT 2012, 2012.

23. D. Micciancio and C. Peikert. Hardness of SIS and LWE with Small Parameters. In CRYPTO 2013, 2013.

24. D. Micciancio and O. Regev. Worst-Case to Average-Case Reductions Based on Gaussian Measures. SIAM Journal on Computing, 37(1), 2007.

25. D. Naor, M. Naor, and J. Lotspiech. Revocation and tracing schemes for stateless receivers. In CRYPTO 2001, 2001.

26. M. Naor and K. Nissim. Certificate revocation and certificate update. In 7 th USENIX Security Symposium, 1998.

27. M. Naor and A. Ziv. Primary-secondary-resolver membership proof systems. In TCC, 2015.

28. R. Ostrovsky, C. Rackoff, and A. Smith. Efficient consistency proofs for generalized queries on a committed database. In ICALP 2004, 2004.

29. D. Papadopoulos, S. Papadopoulos, and N. Triandopoulos. Taking authenticated range queries to arbitrary dimensions. In $A C M-C C S, 2014$.

30. C. Papamanthou, R. Tamassia, and N. Triandopoulos. Optimal verification of operations on dynamic sets. In Crypto, 2011.

31. M. Prabhakaran and R. Xue. Statistically hiding sets. In $C T-R S A, 2009$.

32. R. Tamassia. Authenticated data structures. In European Symp. on Algorithms (ESA), 2003. 


\section{A A Generic Construction of Trapdoor Mercurial Commitments}

Catalano et al. 2] described a generic construction of multi-bit TMC TMC from a trapdoor bit commitment TC $=$ (Setup, Commit, Open, Verify, Fake, Equiv) with an associated $\Sigma$-protocol, $\Pi=$ (Start, Finish, Check) for proving knowledge of a witness $d$ that some given commitment $c$ can be opened to 0 .

The algorithms of the trapdoor bit commitment TC are almost identical that of a TMC scheme as the properties of hard commitments are exactly that of regular commitments.

$-(p k, s k) \leftarrow$ TC.Setup $\left(1^{\lambda}\right)$ : Taking security parameter $\lambda$ as input, outputs a public commitment key $p k$ and secret trapdoor $s k$.

$-C \leftarrow$ TC.Com $(p k, M ; R)$ : Taking public key $p k$, message $M$ and random coins $R$ as inputs, outputs a commitment $C$ for $M$.

$-\pi \leftarrow$ TC.Open $(p k, M ; R)$ : Taking public key $p k$, message $M$ and random coins $R$ as inputs, outputs an opening $\pi$ for $C$ of $M$.

- TC.Verify $(p k, M, C, \pi)$ : Taking public key $p k$, message $M$, commitment $C$ and opening $\pi$ as inputs, accepts if $\pi$ proves that $C$ is a valid commitment to $M$ and rejects otherwise.

$-C \leftarrow \operatorname{Fake}(p k ; R)$ : Taking public key $p k$ and random coins $R$ as inputs, outputs a "fake" commitment $C$ that is initially not tied to any message.

$-\pi \leftarrow \operatorname{TC}$.Equiv $(s k, M ; R)$ : Taking secret key $m s k$, message $M$ and random coins $R$, outputs a supposedly valid opening $\pi$ (fake) of the fake commitment $C=\operatorname{Fake}(p k ; R)$ to $M$.

Let CommitsZero $=\{(c, d) \mid c=\mathrm{TC} . \operatorname{Com}(p k, 0 ; d)\}$ be the NP-relation of the statement " $c$ is a valid commitment to 0 " with $d$ as the witness. The $\Sigma$ protocol $\Pi$ is a three move protocol between a PPT prover $P$ with input $(c, d) \in$ CommitsZero and PPT verifier $V$ with input $c$ which follows the form in Figure2,

$-a \leftarrow \operatorname{Start}\left(c, d ; r_{P}\right)$ : Taking acommitment $c$, witness $d$ that it is a valid commitment to 0 and random coins $r_{P}$, output $a$, a first message of $\Pi$.

$-z \leftarrow \operatorname{Finish}\left(c, d, C h ; r_{P}\right)$ : Taking acommitment $c$, witness $d$, challenge $C h$ and random coins $r_{P}$, output the correct reponse $z$ to the challenge $C h$ with first message $a$.

- Check $(c, a, C h, z)$ : Taking a commitment $c$, first message $a$, challenge $C h$ and response $z$, accepts if the algorithm is convinced that $(c, d) \in$ CommitsZero, i.e. $c=\mathrm{TC} . \operatorname{Com}(p k, 0 ; d)$.

The $\Sigma$-protocol $\Pi$ has to satisfy the following three properties:

- Completeness: If $(c, d) \in$ CommitsZero, then Check $(c, a, C h, z)$ accepts with overwhelming probability.

- Special Soundness: There exists a PPT algorithm, Ext, also known as the knowledge extractor, such that it is (computationally) infeasible to generate $\left(c, a, C h, z, C h^{\prime}, z^{\prime}\right)$, where $C h \neq C h^{\prime} \in \mathcal{M}$, both $\operatorname{Check}(c, a, C h, z)$ and Check $\left(c, a, C h^{\prime}, z^{\prime}\right)$ accept but $\operatorname{Ext}\left(c, a, C h, z, C h^{\prime}, z^{\prime}\right)$ fails to output a valid witness $d$ such that $(c, d) \in$ CommitsZero. 


\begin{tabular}{|lll|}
\hline Prover, $P$ & & Verifier, $V$ \\
\hline$a \leftarrow \operatorname{Start}\left(c, d ; r_{P}\right)$ & $a$ & \\
\cline { 2 - 3 } & $\longleftarrow$ & $C h \leftarrow \mathcal{M}$ \\
$z \leftarrow \operatorname{Finish}\left(c, d, C h ; r_{P}\right)$ & $z$ & Check $(c, a, C h, z)$ \\
\hline
\end{tabular}

Fig. 2. The $\Sigma$ Protocol for CommitsZero, $\Pi$.

- Special Honest-Verifier Zero-Knowledge (HVZK): There exists a PPT algorithm Simul, called the simulator, such that for any $(c, d) \in$ CommitsZero and any fixed challenge $C h$, the following two distributions of transcripts $\{(a, z)\}$ are (computationally) indistinguishable. The first is the distribution of transcripts obtained by running an honest $\mathrm{P}$, with fresh randomness each time, against a verifier with fixed challenge $C h$. The second is the distribution of transcripts output by the simulator $(a, z) \leftarrow \operatorname{Simul}\left(c, C h ; r_{S}\right)$ with fresh randomness $r_{S}$.

- (Strongly Hiding w.r.t. Instance Generation Procedure P) In particular for multi-bit TMC, the special HVZK property has to hold even if $P$ produces $(p k, c, d, \mathcal{I})$, where $p k$ is the public key for TC, $c$ is the input commitment, $d$ is its witness and $\mathcal{I}$ is some auxiliary information given to the adversary. In this case, the distinguisher is given a tuple $(\mathcal{I}, p k, c, a, C h, z)$ where $(a, z)$ is either a valid transcript or output by the simulator.

With this construction, the challenge space of $\Pi$ is the message space of the resulting TMC scheme. Roughly speaking, a commitment in TMC has two parts, a commitment to a bit $c$ and the first message $a$ of a transcript of $\Pi$ proving that $c$ can be opened to 0 . To hard-commit to a message $m$, the committer sets $c=c_{1}=\mathrm{TC}$.Commit $\left(1 ; R_{1}\right)$ and uses $\left(a_{1}, z_{1}\right) \leftarrow \Pi$.Simul $\left(c, m ; r_{c}\right)$ to produce a fake transcript $a_{1}$ proving that $c$ can be opened to 0 . A soft commitment, on the other hand, has $c=c_{0}=\mathrm{TC}$.Commit $\left(0 ; R_{0}\right)$ and $a_{0}=\Pi$.Start $\left(c, R_{0} ; r_{c}\right)$.

Hard openings to a TMC hard commitment $\left(c_{1}, a_{1}\right)$ is made up of an opening of $c_{1}$ to 1 and the second part of the fake transcript that proves $c_{1}$ can be opened to 0 . A soft opening to a TMC commitment $(c, a)$ to a message $m$ is simply the second part of the transcript $(a, z)$ of $\Pi$. Any hard commitment has only one valid $z$, from $(a, z) \leftarrow \Pi$.Simul $\left(c, m ; r_{c}\right)$ and so cannot be soft-opened to any other message $m^{\prime}$ without violating the security of $\Pi$. With a proper commitment to 0 in the first part, a soft commitment can be soft-opened to any message $m$ since we can run $z \leftarrow \Pi$.Finish $\left(c=c_{0}, R_{0}, m ; r_{c_{0}}\right)$.

In TMC, fake commitments and equivocations are straightforward. A TMC fake commitment $(c, a)$ consists of a fake commitment $c=\mathrm{TC} . F a k e\left(R_{F}\right)$ and correct first message $a=\Pi$.Start $\left(c, d_{0} ; r_{c}\right)$, where $d_{0}=\operatorname{Equiv}\left(0 ; R_{F}\right)$ is its fake 
opening to 0 . With $d_{0}$, a fake soft opening is created in the same manner as a real soft opening. To fake hard-open $(c, a)$ to a message $m$, we compute the fake opening $d_{1}=\operatorname{Equiv}\left(1 ; R_{f}\right)$ and correct last message $z \leftarrow \Pi$. Finish $\left(c, d_{0}, m ; r_{c}\right)$.

$-(m p k, m s k) \leftarrow \operatorname{Setup}\left(1^{\lambda}\right)$ : Taking security parameter $\lambda$ as input, run the setup algorithm for TC, $(p k, s k) \leftarrow$ TC.Setup, and output $m p k=p k$ and $m s k=s k$.

- $C \leftarrow \mathbb{H}$ Commit $\left(m p k, M ;\left(R_{1}, r_{c_{1}}\right)\right)$ : Taking public key $m p k$, message $M$ and random coins $\left(R_{1}, r_{c_{1}}\right)$ as inputs, compute $c_{1} \leftarrow \mathrm{TC} \cdot \operatorname{Com}\left(m p k, 1 ; R_{1}\right)$ and $\left(a_{1}, z_{1}\right) \leftarrow \Pi$.Simul $\left(c_{1}, M ; r_{c_{1}}\right)$ and output $C=\left(c_{1}, a_{1}\right)$.

$-\pi \leftarrow \mathbb{H}$ Open $\left(m p k, M ;\left(R_{1}, r_{c_{1}}\right)\right)$ : Taking public key $m p k$, message $M$ and random coins $\left(R_{1}, r_{c_{1}}\right)$ as inputs, compute $\pi_{1} \leftarrow \operatorname{Open}\left(m p k, 1 ; R_{1}\right)$ and $\left(a_{1}, z_{1}\right) \leftarrow \Pi$.Simul $\left(c_{1}, M ; r_{c_{1}}\right)$ and output $\pi=\left(\pi_{1}, z_{1}\right)$.

- $\mathbb{H}$ Verify $(m p k, M, C, \pi)$ : Taking public key $m p k$, message $M$, commitment $C=\left(c_{1}, a_{1}\right)$ and hard opening $\pi=\left(\pi_{1}, z_{1}\right)$ as inputs, accepts if both TC.Verify $\left(m p k, M, c_{1}, \pi_{1}\right)$ and $\Pi$.Check $\left(c_{1}, a_{1}, M, z_{1}\right)$ accepts.

$-C \leftarrow \mathbb{S C o m m i t}\left(m p k ;\left(R_{0}, r_{c_{0}}\right)\right)$ : Taking public key $m p k$ and random coins $\left(R_{0}, r_{c_{0}}\right)$ as inputs, run $c_{0}=\mathrm{TC} . \operatorname{Com}\left(m p k, 0 ; R_{0}\right), a_{0} \leftarrow \Pi$.Start $\left(c_{0}, R_{0} ; r_{c_{0}}\right)$ and output $C=\left(c_{0}, a_{0}\right)$.

$-\tau \leftarrow \mathbb{S O p e n}(m p k, M$, flag; $R)$ : Taking public key $m p k$, message $M$, flag flag and random coins $R$ as inputs:

- If flag $=\mathbb{H}$, we have $R=\left(R_{1}, r_{c_{1}}\right)$ and $c_{1}=\mathrm{TC} \cdot \operatorname{Com}\left(m p k, 1 ; R_{1}\right)$. Run $\left(a_{1}, z_{1}\right) \leftarrow \Pi$.Simul $\left(c_{1}, M ; r_{c_{1}}\right)$ and output $\tau=z_{1}$.

- Otherwise, flag $=\mathbb{S}, R=\left(R_{0}, r_{c_{0}}\right)$ and $c_{0}=\mathrm{TC} \cdot \operatorname{Com}\left(m p k, 0 ; R_{0}\right)$. Run $z_{0} \leftarrow \Pi$.Finish $\left(c_{0}, R_{0}, M ; r_{c_{0}}\right)$ and output $\tau=z_{0}$.

- $\operatorname{SVerify}(m p k, M, C, \tau)$ : Taking public key $m p k$, message $M$, commitment $C=$ $(c, a)$ and soft opening $\tau=z$, accepts if $\Pi$.Check $(c, a, C h, z)$ accepts.

$-C \leftarrow \operatorname{MFake}\left(m s k ;\left(R_{F}, r_{c_{F}}\right)\right)$ : Taking public key $m p k$ and random coins $\left(R_{F}, r_{c_{F}}\right)$ as inputs, compute $c_{F} \leftarrow$ TC.Fake $\left(m p k ; R_{F}\right)$, an equivocation of $c_{F}$ to $0, R_{0, F} \leftarrow$ TC.Equiv $\left(m s k, 0 ; R_{F}\right)$ and $a_{F} \leftarrow \Pi$.Start $\left(c_{F}, R_{0, F} ; r_{c_{F}}\right)$. Output $C=\left(c_{F}, a_{F}\right)$.

$-\pi \leftarrow \mathbb{H}$ Equivocate $\left(m s k, M ;\left(R_{F}, r_{c_{F}}\right)\right)$ : Taking secret key $m s k$, message $M$ and random coins $R$, equivocate $c_{F}$ to 0 with $R_{0, F} \leftarrow$ TC.Equiv $\left(m s k, 0 ; R_{F}\right)$, compute $z_{F} \leftarrow \Pi$.Finish $\left(c_{F}, R_{0, F}, M ; r_{c_{F}}\right)$ and $\pi_{F} \leftarrow$ TC.Equiv $\left(m s k, 1 ; R_{F}\right)$. Output $\pi=\left(\pi_{F}, z_{F}\right)$.

$-\tau \leftarrow$ SEquivocate $\left(m s k, M ;\left(R_{F}, r_{c_{F}}\right)\right)$ : Taking secret key $m s k$, message $M$ and random coins $R$, equivocate $c_{F}$ to 0 with $R_{0, F} \leftarrow \mathrm{TC}$.Equiv $\left(m s k, 0 ; R_{F}\right)$, compute $z_{F} \leftarrow \Pi$.Finish $\left(c_{F}, R_{0, F}, M ; r_{c_{F}}\right)$ and output $\tau=z_{F}$.

We re-state the security properties of the generic construction of Catalano et al. 2] in the following theorem without proof and refer interested readers to [2, Section 5].

Theorem 3. The construction above is correct, satisfies mercurial-hiding and mercurial-binding and the $\mathrm{HH}, \mathrm{HS}$, SS equivocation properties.

Here, we propose our extensions to the generic construction to support the explanation algorithms required for range proofs. 
- $R \leftarrow \operatorname{Explain}\left(m p k ;\left(R_{0} ; r_{c_{0}}\right)\right)$ : On input of the public commitment key $m p k$ and random coins $\left(R_{0} ; r_{c_{0}}\right)$ such that $C=\left(c_{0}=\mathrm{TC} \cdot \operatorname{Com}\left(m p k, 0 ; R_{0}\right), a_{0}=\right.$ $\Pi$.Start $\left.\left(c_{0}, R_{0} ; r_{c_{0}}\right)\right)$, outputs $R=\left(R_{0}, r_{c_{0}}\right)$.

- EVerify $\left(m p k, C,\left(R_{0} ; r_{c_{0}}\right)\right)$ : On input of the public commitment key $m p k$, a commitment $C=(c, a)$ and random coins $\left(R_{0} ; r_{c_{0}}\right)$, this algorithm accepts if $c=\mathrm{TC} \cdot \operatorname{Com}\left(m p k, 0 ; R_{0}\right)$ and $a=\Pi$.Start $\left(c, R_{0} ; r_{c_{0}}\right)$.

- FakeExplain $\left(m s k ;\left(R_{F}, r_{c_{F}}\right)\right)$ : On input of the public commitment key $m p k$ and random coins $\left(R_{F}, r_{c_{F}}\right)$ such that $C=\left(c_{F}, a_{F}\right) \leftarrow \operatorname{MFake}\left(m p k ;\left(R_{F}, r_{c_{F}}\right)\right)$, this algorithm outputs random coins $R^{\prime}=\left(R_{0, F}, r_{c_{F}}\right)$ such that $c_{F}=$ $\mathbb{S C o m m i t}\left(m p k ; R_{0, F}\right)$ and $a_{F}=\Pi$.Start $\left(c_{F}, R_{0, F} ; r_{c_{F}}\right)$.

Now, we show that this construction satisfies the newly introduced softexplain (SE) equivocation property as well.

Theorem 4. The construction above satisfies the SE equivocation property.

Proof. Fake commitments and soft commitments are indistinguishable since TC satisfies the equivocation property and the second part of both commitments are output of the same algorithm $\Pi$.Start. We show that the real and fake explanations are computationally indistinguishable below.

The random coins needed to generate a soft commitment consist of the random coin used to commit to $0, R_{0}$, and some randomness required for the $\Sigma$ protocol $\Pi, r_{c_{0}}$. Since the trapdoor commitment scheme TC satisfies the equivocation property, the tuples $\left(0, \mathrm{TC} . \operatorname{Com}\left(m p k, 0 ; R_{0}\right)\right.$, TC.Open $\left.\left(m p k, 0 ; R_{0}\right)\right)$ and $\left(0\right.$, TC.Fake $\left(m s k ; R_{F}\right)$, TC.Equiv $\left.\left(m s k, 0 ; R_{F}\right)\right)$ are computationally indistinguishable. In almost all known trapdoor commitments, TC.Open $\left(m p k, 0 ; R_{0}\right)$ outputs $R_{0}$ itself. Therefore, the equivocation algorithm produces $R_{0, F}$ that is computationally indistinguishable from $R_{0}$.

As for the random coins used in $\Pi$, both soft and fake commitments use randomness drawn from the same distribution, $r_{c_{0}}$ and $r_{c_{F}}$, to compute $\Pi$.Start. Hence, the second part of the random coins are computationally indistinguishable as well meaning that the output of FakeExplain for fake commitments and Explain for soft commitments are computationally indistinguishable.

\section{B Formal Description of ProveRQ and VerifyRQ}

We give the formal description of the range query proof generation and verification algorithms, ProveRQ and VerifyRQ. Recall that the algorithms for opening paths, Steiner trees and intervals take a database commitment $\operatorname{com}_{\mathrm{D}}$ and its associated decommitment information $\Delta_{\mathrm{D}}$ as inputs. In the following, we note that the decommitment information for a ZK-EEDB commitment $\Delta$ encompasses the decommitment information for every commitment computed in the ZK-EEDB ComDB algorithm. For simplicity, we input $\Delta$ instead of specifying the particular decommitment information required for each commitment.

$$
-\Pi_{\mathfrak{R}} \leftarrow \operatorname{ProveRQ}(\text { crs },(\text { com, } \Delta), \mathfrak{R}): \text { Let } \mathfrak{R}=\left[a_{x}, b_{x}\right] \times\left[a_{y}, b_{y}\right] .
$$


- Either $\left[a_{x}, b_{x}\right]=\left[0,2^{\ell}\right)$ or $\left[a_{y}, b_{y}\right]=\left[0,2^{\ell}\right)$ :

- If $\left[a_{y}, b_{y}\right]=\left[0,2^{\ell}\right)$, compute $\Pi_{\mathrm{D}} \leftarrow$ Openl $\left(\operatorname{crs},\left(\operatorname{com}_{\mathrm{D}}, \Delta\right),\left[a_{x}, b_{x}\right]\right)$ and let $\mathcal{L} \leftarrow \operatorname{Verifyl}\left(\operatorname{crs}, \operatorname{com}_{\mathrm{D}},\left[a_{x}, b_{x}\right], \Pi_{\mathrm{D}}\right)$. For each $(x, y) \in \mathcal{L}$ :

1. Compute $\Pi_{x, \mathrm{D}_{y}^{-1}} \leftarrow \mathbb{H}$ OpenPath $\left(\right.$ crs $\left.,\left(\operatorname{com}_{\mathrm{D}_{y}^{-1}}, \Delta\right), x\right)$.

2. Compute $\Pi_{x, \mathrm{D}^{-1}} \leftarrow \mathbb{H}$ OpenPath $\left(\right.$ crs $\left.,\left(\operatorname{com}_{\mathrm{D}^{-1}}, \Delta\right), y\right)$.

Set $\Pi_{\mathrm{D}^{-1}}=\left\{\Pi_{x, \mathrm{D}_{y}^{-1}}, \Pi_{x, \mathrm{D}^{-1}}\right\}_{(x, y) \in \mathcal{L}}$ and nil if $\mathcal{L}=\emptyset$.

- If $\left[a_{x}, b_{x}\right]=\left[0,2^{\ell}\right)$, compute $\Pi_{\mathrm{D}^{-1}}^{\prime} \leftarrow \operatorname{Openl}\left(\operatorname{crs},\left(\operatorname{com}_{\mathrm{D}^{-1}}, \Delta\right),\left[a_{y}, b_{y}\right]\right)$ and let $\mathcal{L}^{\prime} \leftarrow \operatorname{Verifyl}\left(\right.$ crs $\left., \operatorname{com}_{\mathrm{D}^{-1}},\left[a_{y}, b_{y}\right], \Pi_{\mathrm{D}^{-1}}^{\prime}\right)$. If $\mathcal{L}^{\prime}=\emptyset$, then set $\Pi_{\mathrm{D}}=$ nil and $\Pi_{\mathrm{D}^{-1}}=\Pi_{\mathrm{D}^{-1}}^{\prime}$ and skip to the return statement. Otherwise, set $\mathcal{L}=\emptyset$ and, for each $\left(y, \operatorname{com}_{\mathrm{D}_{y}^{-1}}\right) \in \mathcal{L}^{\prime}$ :

1. Compute $\Pi_{y} \leftarrow$ Openl $\left(\operatorname{crs},\left(\operatorname{com}_{\mathrm{D}_{y}^{-1}}, \Delta\right),\left[a_{x}, b_{x}\right]\right)$ and let the set $\mathcal{L}_{y} \leftarrow \operatorname{Verifyl}\left(\right.$ crs $\left., \operatorname{com}_{\mathrm{D}_{y}^{-1}},\left[0,2^{\ell}\right), \Pi_{y}\right)$.

2. For each $(x, 1) \in \mathcal{L}_{y}$, set $\mathcal{L}=\mathcal{L} \cup\{(x, y)\}$ and compute the proof $\Pi_{x} \leftarrow \mathbb{H}$ OpenPath $\left(\right.$ crs $\left.,\left(\operatorname{com}_{\mathrm{D}}, \Delta\right), x\right)$.

Set $\Pi_{\mathrm{D}}=\left\{\Pi_{x}\right\}_{x \in[\mathcal{L}]}$ and $\Pi_{\mathrm{D}^{-1}}=\left(\Pi_{\mathrm{D}^{-1}}^{\prime},\left\{\Pi_{y}\right\}_{\left(y, c o m_{\mathrm{D}_{y}^{-1}}\right) \in \mathcal{L}^{\prime}}\right)$.

- Otherwise, $\left[a_{x}, b_{x}\right] \neq\left[0,2^{\ell}\right)$ and $\left[a_{y}, b_{y}\right] \neq\left[0,2^{\ell}\right)$.

Step 1 (Handle $\operatorname{com}_{\mathrm{D}^{-1}}$ ). For each value $y \in\left[a_{y}, b_{y}\right]$ :

If $\mathrm{D}_{y}^{-1}=\emptyset$ :

a. Compute $\Pi_{\mathrm{D}_{y}^{-1}} \leftarrow$ SOpenPath $\left(\right.$ crs $\left.,\left(\operatorname{com}_{\mathrm{D}^{-1}}, \Delta\right), y\right)$ and let $\Pi_{\mathrm{D}_{y}^{-1}}=\left(\left\{C_{\left.y\right|_{j}}, C_{\left(\left.y\right|_{j}\right)^{\prime}}\right\}_{1 \leq j \leq \ell},\left\{\tau_{\left.y\right|_{j}}\right\}_{0 \leq j \leq \ell-1}\right)$, ignoring the value $\perp$ at $C_{y}$ and its proof $\tau_{y}$.

b. Compute $\operatorname{com}_{\mathrm{D}_{y}^{-1}} \leftarrow \mathbb{S}$ Commit $\left(\right.$ crs $\left.; R_{\mathrm{D}_{y}^{-1}}\right)$ and new soft decommitment $\tau_{y} \leftarrow$ SOpen $\left(\right.$ crs, com $\left._{\mathrm{D}_{y}^{-1}} ; R_{\mathrm{D}_{y}^{-1}}\right)$.

c. Compute $\Pi_{\mathrm{D}_{y}^{-1},\left[a_{x}, b_{x}\right]} \leftarrow$ OpenI $\left(\operatorname{crs},\left(\operatorname{com}_{\mathrm{D}_{y}^{-1}}, \Delta\right),\left[a_{x}, b_{x}\right]\right)$.

Set $\Pi_{y, \mathrm{D}^{-1}}=\left(\Pi_{\mathrm{D}_{y}^{-1}}, \tau_{y}, \operatorname{com}_{\mathrm{D}_{y}^{-1}}, \Pi_{\mathrm{D}_{y}^{-1},\left[a_{x}, b_{x}\right]}\right)$.

If $\mathrm{D}_{y}^{-1} \neq \emptyset$ :

a. Compute $\Pi_{\mathrm{D}_{y}^{-1}} \leftarrow \mathbb{H}$ OpenPath $\left(\operatorname{crs},\left(\operatorname{com}_{\mathrm{D}^{-1}}, \Delta\right), y\right)$ and let $\Pi_{\mathrm{D}_{y}^{-1}}=\left(\operatorname{com}_{\mathrm{D}_{y}^{-1}},\left\{C_{\left.y\right|_{j}}, C_{\left(\left.y\right|_{j}\right)^{\prime}}\right\}_{1 \leq j \leq \ell},\left\{\pi_{\left.y\right|_{j}}\right\}_{0 \leq j \leq \ell-1}\right)$.

b. Compute $\Pi_{\mathrm{D}_{y}^{-1},\left[a_{x}, b_{x}\right]} \leftarrow$ Openl $\left(\operatorname{crs} s,\left(\operatorname{com}_{\mathrm{D}_{y}^{-1}}, \Delta\right),\left[a_{x}, b_{x}\right]\right)$.

Set $\Pi_{y, \mathrm{D}^{-1}}=\left(\Pi_{\mathrm{D}_{y}^{-1}}, \pi_{y}, \operatorname{com}_{\mathrm{D}_{y}^{-1}}, \Pi_{\mathrm{D}_{y}^{-1},\left[a_{x}, b_{x}\right]}\right)$.

Set $\Pi_{\mathrm{D}^{-1}}=\left\{\Pi_{y, \mathrm{D}^{-1}}\right\}_{y \in\left[a_{y}, b_{y}\right]}$.

Step 2 (Consistency with $\operatorname{com}_{\mathrm{D}}$ ). Let $\mathcal{L}^{\prime}=\emptyset$.

a. For each $y \in\left[a_{y}, b_{y}\right]$, compute the set of keys in $\left[a_{x}, b_{x}\right]$ with value $y$ with $\mathcal{L}_{y} \leftarrow \operatorname{Verifyl}\left(\right.$ crs $\left., \operatorname{com}_{\mathrm{D}_{y}^{-1}},\left[a_{x}, b_{x}\right], \Pi_{\mathrm{D}_{y}^{-1},\left[a_{x}, b_{x}\right]}\right)$ and set $\mathcal{L}^{\prime}=\mathcal{L}^{\prime} \cup\{(x, y)\}$ for each $(x, 1) \in \mathcal{L}^{\prime}$.

b. If $\mathcal{L}^{\prime}=\emptyset$, set $\Pi_{\mathrm{D}}=$ nil and skip to the return statement.

c. Otherwise, for $(x, y) \in \mathcal{L}^{\prime}$, compute the proof that $y$ is committed at leaf $x$ of $\operatorname{com}_{\mathrm{D}}, \Pi_{x} \leftarrow \mathbb{H}$ OpenPath $\left(\operatorname{crs},\left(\operatorname{com}_{\mathrm{D}}, \Delta_{\mathrm{D}}\right), x\right)$. 
If $\Pi_{\mathrm{D}} \neq n i l$, set $\Pi_{\mathrm{D}}=\left\{\Pi_{x}\right\}_{x \in[\mathcal{L}]}$.

Return $\Pi_{\mathfrak{R}}=\left(\Pi_{\mathrm{D}}, \Pi_{\mathrm{D}^{-1}}\right)$ and add any random coins used to $\Delta$.

$-\mathcal{L} \leftarrow \operatorname{VerifyRQ}\left(\right.$ crs, com, $\left.\mathfrak{R}, \Pi_{\mathfrak{R}}\right)$ : Let $\mathfrak{R}=\left[a_{x}, b_{x}\right] \times\left[a_{y}, b_{y}\right]$ and its proof $\Pi_{\mathfrak{R}}=\left(\Pi_{\mathrm{D}}, \Pi_{\mathrm{D}^{-1}}\right)$. Note that the algorithm rejects by outputting $\mathcal{L}=$ bad.

- If $\left[a_{y}, b_{y}\right]=\left[0,2^{\ell}\right)$ : Compute $\mathcal{L} \leftarrow \operatorname{Verifyl}\left(\right.$ crs $\left., \operatorname{com}_{\mathrm{D}},\left[a_{x}, b_{x}\right], \Pi_{\mathrm{D}}\right)$. If the result is $\mathcal{L}=b a d$, return bad. If $\mathcal{L}=\emptyset$ and $\Pi_{\mathrm{D}^{-1}}=$ nil, skip to the return statement. Otherwise, let the proofs for $\mathrm{D}^{-1}$ be $\Pi_{\mathrm{D}^{-1}}=$ $\left\{\Pi_{x, \mathrm{D}^{-1}}, \Pi_{x, \mathrm{D}_{y}^{-1}}\right\}_{(x, y) \in \mathcal{L}}$ and return bad if they do not parse properly. For $(x, y) \in \mathcal{L}$ :

1. Compute ans $\leftarrow$ VerifyPath $\left(\right.$ crs $\left., \operatorname{com}_{\mathrm{D}^{-1}}, y, \Pi_{x, \mathrm{D}^{-1}}\right)$ return bad in the event that ans $=$ bad.

2. Otherwise, compute ans $\leftarrow$ VerifyPath $\left(\right.$ crs , ans, $\left.x, \Pi_{x, \mathrm{D}_{y}^{-1}}\right)$ and return bad if $a n s^{\prime}=$ bad or $a n s^{\prime} \neq 1$.

If none of the steps returned bad, output $\mathcal{L}$.

- If $\left[a_{x}, b_{x}\right]=\left[0,2^{\ell}\right)$ : Let $\Pi_{\mathfrak{R}}=\left(\Pi_{\mathrm{D}}, \Pi_{\mathrm{D}^{-1}}\right)$ and $\mathcal{L}=\emptyset$.

○ If $\Pi_{\mathrm{D}^{-1}}=\Pi$ :

1. Compute $\mathcal{L}^{\prime} \leftarrow \operatorname{Verifyl}\left(\right.$ crs $\left., \operatorname{com}_{\mathrm{D}^{-1}},\left[a_{y}, b_{y}\right], \Pi\right)$.

2. If $\mathcal{L}^{\prime}=\emptyset$ and $\Pi_{\mathrm{D}}=$ nil, skip to the return statement; otherwise, return bad.

○ Otherwise, $\Pi_{\mathrm{D}^{-1}}=\left(\Pi_{\mathrm{D}^{-1}}^{\prime},\left\{\Pi_{y_{i}}\right\}_{i=1}^{p}\right)$ for some $p \geq 1$.

1. Compute $\mathcal{L}^{\prime} \leftarrow \operatorname{Verifyl}\left(\right.$ crs $\left., \operatorname{com}_{\mathrm{D}^{-1}},\left[a_{y}, b_{y}\right], \Pi_{\mathrm{D}^{-1}}^{\prime}\right)$ and return bad if $\mathcal{L}^{\prime}=$ bad or $\emptyset$.

2. Otherwise, $\mathcal{L}^{\prime}=\left\{\left(y_{i}, \operatorname{com}_{y_{i}}\right)\right\}_{i=1}^{p^{\prime}}$ and return bad if $p^{\prime} \neq p$.

3. For each $\left(y, \operatorname{com}_{y}\right) \in \mathcal{L}^{\prime}$ :

a. Compute $\mathcal{L}_{y} \leftarrow \operatorname{Verifyl}\left(\mathrm{crs}, \operatorname{com}_{y},\left[0,2^{\ell}\right), \Pi_{y}\right)$ and return bad if $\mathcal{L}_{y}=$ bad or $\emptyset$.

b. Set $\mathcal{L}=\mathcal{L} \cup\{(x, y)\}$ for each $(x, 1) \in \mathcal{L}_{y}$.

4. Let $\Pi_{\mathrm{D}}=\left\{\Pi_{x_{i}}\right\}_{i=1}^{p^{\prime \prime}}$ and return bad if $|\mathcal{L}| \neq p^{\prime \prime}$.

5. For each $(x, y) \in \mathcal{L}$, compute ans $\leftarrow$ VerifyPath $\left(\right.$ crs $\left., \operatorname{com}_{\mathrm{D}}, x, \Pi_{x}\right)$ and returned bad if ans $\neq y$.

Return $\mathcal{L}$ if none of the steps returned bad.

- Otherwise, $\left[a_{x}, b_{x}\right] \neq\left[0,2^{\ell}\right)$ and $\left[a_{y}, b_{y}\right] \neq\left[0,2^{\ell}\right)$. Let $\Pi_{\mathfrak{R}}=\left(\Pi_{\mathrm{D}}, \Pi_{\mathrm{D}^{-1}}\right)$ and $\Pi_{\mathrm{D}^{-1}}=\left\{\Pi_{y, \mathrm{D}^{-1}}\right\}_{y \in\left[a_{y}, b_{y}\right]}$. Reject if $\Pi_{\mathrm{D}^{-1}}$ is not of this form.

1. For each $y \in\left[a_{y}, b_{y}\right]$, let $\Pi_{y, \mathrm{D}^{-1}}=\left(\Pi_{\mathrm{D}_{y}^{-1}}, \eta_{y}, \operatorname{com}_{\mathrm{D}_{y}^{-1}}, \Pi_{\mathrm{D}_{y}^{-1},\left[a_{x}, b_{x}\right]}\right)$.

a. Compute ans $_{y} \leftarrow$ VerifyPath $\left(\right.$ crs $\left., \operatorname{com}_{\mathrm{D}^{-1}}, y, \Pi_{\mathrm{D}_{y}^{-1}}\right)$.

b. Compute $a n s_{y}^{\prime}=\mathbb{S V}$ erify $\left(\right.$ crs $\left., a n s_{y}, \operatorname{com}_{\mathrm{D}_{y}^{-1}}, \eta_{y}\right)$ if $\eta_{y}$ is a soft decommitment and ans $s^{\prime}=\mathbb{H}$ Verify $\left(\right.$ crs, ans $\left._{y}, \operatorname{com}_{\mathrm{D}_{y}^{-1}}, \eta_{y}\right)$ if $\eta_{y}$ is a hard decommitment.

c. Return if verification fails; otherwise, continue.

d. Compute $\mathcal{L}_{y} \leftarrow \operatorname{Verifyl}\left(\right.$ crs $\left., a n s_{y}^{\prime},\left[a_{x}, b_{x}\right], \Pi_{\mathrm{D}_{y}^{-1},\left[a_{x}, b_{x}\right]}\right)$.

e. If $\mathcal{L}_{y}=$ bad, return bad. Otherwise, set $\mathcal{L}=\mathcal{L} \cup \mathcal{L}_{y}$. 
2. If $\mathcal{L}=\emptyset$ and $\Pi_{\mathrm{D}}=n i l$, skip to the return statement.

3. Otherwise, $\Pi_{\mathrm{D}}=\left\{\Pi_{x}\right\}_{i=1}^{p}$ for some $p \geq 1$ and reject if $|\mathcal{L}| \neq p$.

4. For each $(x, y) \in \mathcal{L}$, compute ans $\leftarrow \operatorname{VerifyPath}\left(\right.$ crs $\left., \operatorname{com}_{\mathrm{D}}, x, \Pi_{x}\right)$ and return bad if ans $\neq y$.

Return $\mathcal{L}$.

\section{Expressive Queries in ZK-EEDB}

In this section. we formally describe the expressive queries mentioned in Section 4.4. The ability to prove range queries in zero-knowledge enables these expressive queries in ZK-EEDB. Furthremore, the introduction of the second Merkle tree committing to the "reversed database" $\mathrm{D}^{-1}$ allows queries over values and records and even sub-databases $\mathrm{D}^{-1}(y)$ of the committed database, $\mathrm{D}$.

Leveraging range queries over keys, values and records, we showcase several advanced queries that can be answered efficiently in zero-knowledge: nearest neighbour, minimum/maximum and $k$-minimum/maximum queries. First, we have to decide how the records of a database are ordered. There are two main orders that can be used, the lexicographic order (LO) and reverse lexicographic order (RLO):

- LO: $\left(x^{\prime}, y^{\prime}\right)<_{L O}(x, y)$ if $\left(x^{\prime}<x\right.$ or $x^{\prime}=x$ and $y^{\prime}<y$.

- RLO: $\left(x^{\prime}, y^{\prime}\right)<_{R L O}(x, y)$ if $y^{\prime}<y$ or $y^{\prime}=y$ and $x^{\prime}<x$.

To showcase the full capabilities of ZK-EEDB, we focus on RLO below.

\section{C.1 Nearest Neighbour Queries}

- Nearest Neighbour (NN) (Key): Given a key $x$, return the record $\left(x^{\prime}, \mathrm{D}\left(x^{\prime}\right)\right)$ such that $\mathrm{D}\left(x^{*}\right)=\perp$ for all $x^{*}$ where $\left|x^{*}-x\right| \leq\left|x^{\prime}-x\right|$ or $\emptyset$ if $\mathrm{D}=\emptyset$.

- Nearest Neighbour (Value): Given a value $y$, return the set $\mathbf{D}^{-1}\left(y^{\prime}\right)$ such that $\mathrm{D}^{-1}\left(y^{*}\right)=\emptyset$ for all $y^{*}$ where $\left|y^{*}-y\right| \leq\left|y^{\prime}-y\right|$ or $\emptyset$ if $\mathrm{D}=\emptyset$.

- RLO Nearest Neighbour (RLO-NN) (Record): Given a record $(x, y)$, return the record $\left(x^{\prime}, \mathrm{D}\left(x^{\prime}\right)\right)$ where either

a. $\mathrm{D}\left(x^{\prime}\right)=y$ and $\mathrm{D}\left(x^{*}\right) \neq y$ for all $x^{*}$ such that $\left|x^{*}-x\right| \leq\left|x^{\prime}-x\right|$; or

b. $\mathrm{D}\left(x^{\prime}\right) \neq y$ and $\nexists x^{*} \in\left[0,2^{\ell}\right)$ such that $\left|\mathrm{D}\left(x^{*}\right)-y\right| \leq\left|\mathrm{D}\left(x^{\prime}\right)-y\right|$.

In an NN query over keys, one inputs a key $x$ and expects the nearest key $x^{\prime}$ such that there are no other keys $x^{*}$ with $\left|x-x^{*}\right|<\left|x-x^{\prime}\right|$. This problem, also called closest point query, was previously considered by Ghosh et al. [12] who gave a simple method for proving the answers in zero-knowledge in the three-party setting. Their techniques are very simple: Let $x^{\prime}$ be the nearest neighbour of $x$ in the database with value $\mathrm{D}\left(x^{\prime}\right)$ and assume that $x^{\prime}>x$ without loss of generality. Then, the correctness of the answer is shown by proving that $\left(x^{\prime}, \mathrm{D}\left(x^{\prime}\right)\right)$ is the only record in the database whose key is in $\left[2 x-x^{\prime}, x^{\prime}\right]$ using ProveRQ. For ZK-EEDB, we additionally prove consistency between the digests by returning 
a two-tiered hard authentication path, first from leaf $x^{\prime}$ to the root of $\operatorname{com}_{\mathrm{D}_{y}^{-1}}$ and then the leaf $y$ to the root of $\operatorname{com}_{\mathrm{D}^{-1}}$.

Going further, we show that NN queries over values and records are also possible with ZK-EEDB. For NN queries over values, we adapt techniques for NN queries over keys to show that the only value $y^{\prime}$ with $\mathrm{D}^{-1}\left(y^{\prime}\right) \neq \emptyset$ in the interval $\left[2 y-y^{\prime}, y^{\prime}\right]$ (if $y^{\prime}>y$ ), $\left[y^{\prime}, 2 y-y^{\prime}\right]$ (if $y^{\prime}<y$ ) is $y^{\prime}$ using ProveRQ. Then, to ensure consistency of the two digests, we prove that the returned set $\mathrm{D}^{-1}\left(y^{\prime}\right)$ is correctly committed in $\operatorname{com}_{\mathrm{D}}$.

On the other hand, RLO-NN queries over records require ZK-EEDB's ability to prove (non-)membership statements on values of the database. For a query $(x, y)$, the answer $\left(x^{\prime}, \mathrm{D}\left(x^{\prime}\right)\right)$ lies in one of two disjoint sets:

1. $\mathrm{D}\left(x^{\prime}\right) \neq y$ : This defaults to an NN query over values, with the exception that we return the record $\left(x^{\prime}, \mathrm{D}\left(x^{\prime}\right)\right)$ where $x^{\prime}=\max _{\left(x^{*}, 1\right) \in \mathrm{D}^{-1}\left(y^{\prime}\right)} x^{*}$ instead of the entire $\mathrm{D}^{-1}\left(y^{\prime}\right)$. This is accomplished with ProveRQ with the input range $\left[x^{\prime}, 2^{\ell}\right) \times\left[\mathrm{D}\left(x^{\prime}\right), \mathrm{D}\left(x^{\prime}\right)\right]$ to get the correct record and showing that $\mathrm{D}^{-1}(y)=\emptyset$ for all other values $y^{*}$ with $\left|y^{*}-y\right|<\left|\mathrm{D}\left(x^{\prime}\right)-y\right|$.

2. $\mathrm{D}\left(x^{\prime}\right)=y$ : In this case, we perform an NN query over keys on the subdatabase $\mathrm{D}^{-1}(y)$ with input $x$. Then, the two-tiered hard authentication path, first from leaf $x^{\prime}$ to the root of $\operatorname{com}_{\mathrm{D}_{y}^{-1}}$ and then from leaf $y$ to the root of $\operatorname{com}_{\mathrm{D}^{-1}}$, is returned.

In both cases, for consistency between the digests, we prove that the returned record $\left(x^{\prime}, y^{\prime}\right)$ is correctly in $\operatorname{com}_{\mathrm{D}}$ as well.

$k$-Nearest Neighbours Query. Realising the $k$-nearest neighbours $(k \mathrm{NN})$ query with RLO is straightfoward: we simply use the record that is furthest from $x$ to determine the intervals we need for ProveRQ. Let $\left(x_{k}, \mathrm{D}\left(x_{k}\right)\right)$ be the record that is furthest away and assume that $\mathrm{D}\left(x_{k}\right)>y$. Then, we simply run ProveRQ with the input range $\mathfrak{R}=\left[0,2^{\ell}\right) \times\left(2 y-\mathrm{D}\left(x_{k}\right), \mathrm{D}\left(x_{k}\right)\right)$ to show that there are less than $k$ records in this range. Next, at the edges, because there might be more records than $k$ records if we used [2y- $\left.\mathrm{D}\left(x_{k}\right), \mathrm{D}\left(x_{k}\right)\right]$, we instead compute proofs for them separately. If there are some records $\left(x_{i}, \mathrm{D}\left(x_{i}\right)\right)$ with $\mathrm{D}\left(x_{i}\right)=2 y-\mathrm{D}\left(x_{k}\right)$, then we know that $x_{i}>2^{\ell}-x_{k}$. This is because should $x_{i}<2^{\ell}-x_{k}$, then the distance from $(x, y)\left(x_{i}, \mathrm{D}\left(2 y-x_{k}\right)\right)$ is greater than the distance to $\left(x_{i}, \mathrm{D}\left(2 y-x_{k}\right)\right)$, violating our initial assumption that $\left(x_{k}, \mathrm{D}\left(x_{k}\right)\right)$ is the record that is furthest from $(x, y)$. Therefore, we use ProveRQ with the following two ranges $\mathfrak{R}_{1}=\left[2^{\ell}-x_{k}, 2^{\ell}\right) \times\left[2 x-\mathrm{D}\left(x_{k}\right), 2 x-\mathrm{D}\left(x_{k}\right)\right]$ and $\mathfrak{R}_{2}=$ $\left[0, x_{k}\right] \times\left[\mathrm{D}\left(x_{k}\right), \mathrm{D}\left(x_{k}\right)\right]$ to complete the proof of correctness of the $k \mathrm{NN}$ query.

\section{C.2 Minimum/Maximum Query}

- Minimum (resp. Maximum) (Key): Given a range $\mathfrak{R}=\left[a_{x}, b_{x}\right] \times\left[0,2^{\ell}\right)$, return the record $(x, \mathrm{D}(x))$, where $\mathrm{D}(x) \neq \perp$ and $x<x^{\prime}$ (resp. $\left.x>x^{\prime}\right)$ for all $x^{\prime} \in\left[a_{x}, b_{x}\right] \backslash\{x\}$ or $\emptyset$ if $\mathrm{D} \cap \mathfrak{R}=\emptyset$. 
- Minimum (resp. Maximum) (Value): Given a range $\mathfrak{R}=\left[0,2^{\ell}\right) \times\left[a_{y}, b_{y}\right]$, return the set $\mathrm{D}^{-1}(y)$, where $\mathrm{D}_{y}^{-1} \neq \emptyset$ and $y<y^{\prime}$ (resp. $\left.y>y^{\prime}\right)$ for all $y^{\prime} \in\left[a_{y}, b_{y}\right] \backslash\{y\}$ or $\emptyset$ if $\mathrm{D} \cap \mathfrak{R}=\emptyset$.

- RLO Minimum (resp. Maximum) (Record): Given a range $\mathfrak{R}=\left[a_{x}, b_{x}\right] \times$ $\left[a_{y}, b_{y}\right] \subset\left[0,2^{\ell}\right) \times\left[0,2^{\ell}\right)$

If $\mathrm{D} \cap \Re \neq \emptyset$ : return the record $(x, y) \in \mathrm{D}$ such that $(x, y)<_{R L O}\left(x^{\prime}, y^{\prime}\right)$ (resp. $\left.(x, y)>_{R L O}\left(x^{\prime}, y^{\prime}\right)\right)$ for all $\left(x^{\prime}, y^{\prime}\right) \in(\mathrm{D} \cap \Re) \backslash\{(x, y)\}$.

If $\mathrm{D} \cap \mathfrak{R}=\emptyset$ : return $\emptyset$.

For mimimum (resp. maximum) queries over keys, the proof of correctness can be obtained by running ProveRQ with the input range $\left[a_{x}, x\right] \times\left[0,2^{\ell}\right)$ (resp. $\left[x, b_{x}\right] \times\left[0,2^{\ell}\right)$ ). Similarly, for minimum (resp. maximum) queries over values, we run ProveRQ with the input range $\left[0,2^{\ell}\right) \times\left[a_{y}, y\right]\left(\right.$ resp. $\left.\left[0,2^{\ell}\right) \times\left[y, b_{y}\right]\right)$.

The proofs of correctness for RLO minimum (resp. maximum) queries over records are more involved. If $D \cap \mathfrak{R}=\emptyset$, then the proof of the answer is the proof of correctness of the range query with input $\mathfrak{R}$. If there is a minimum (resp. maximum) record $(x, y)$, then we prove that the range $\left[a_{x}, b_{x}\right] \times\left[a_{y}, y\right]$ is empty in the database and the only record in $\left[\mathrm{D}^{-1}(y)\right] \cap\left[a_{x}, x\right]$ is $(x, y)$ (resp. the range $\left[a_{x}, b_{x}\right] \times\left[y, b_{y}\right]$ is empty and the only record in $\left[\mathrm{D}^{-1}(y)\right] \cap\left[x, b_{x}\right]$ is $(x, y))$ using ProveRQ.

From this, we can see that there are some limitations on the range $\mathfrak{R}$ that we can support RLO minimum/maximum queries over records efficiently. The proof of correctness requires showing that a range $\left[a_{x}, b_{x}\right] \times\left[y, b_{y}\right]$ or $\left[a_{x}, b_{x}\right] \times\left[a_{y}, y\right]$ is empty, which will be done by inputting it into ProveRQ. Since ProveRQ can only support ranges where $\left[a_{y}, y\right]$ or $\left[y, b_{y}\right]$ are of polynomial length, we can only prove minimum/maximum queries over records for ranges $\left[a_{x}, b_{x}\right] \times\left[a_{y}, b_{y}\right]$ where $\left[a_{y}, b_{y}\right]$ has polynomial length. The length of $\left[a_{x}, b_{x}\right]$ can be exponential as with range queries.

\section{C.3 $k$-minimum/maximum Elements Query}

- $k$-maximum (resp. minimum) (Key): Given an interval $\left[a_{x}, b_{x}\right]$, return the set of records $\left\{\left(x_{i}, \mathrm{D}\left(x_{i}\right)\right)\right\}_{i=1}^{k}$, where $\mathrm{D}\left(x_{1}\right), \ldots, \mathrm{D}\left(x_{k}\right) \neq \perp$ and $x_{1}>x_{2}>$ $\ldots>x_{k}>x^{\prime}\left(\right.$ resp. $\left.x_{1}<x_{2}<\ldots<x_{k}<x^{\prime}\right)$ for all $x^{\prime} \in\left[a_{x}, b_{x}\right] \backslash\{x\}$.

- $k$-maximum (resp. minimum) (Value): On input of an interval $\left[a_{y}, b_{y}\right]$, return $\bigcup_{i=1}^{k} \mathrm{D}^{-1}\left(y_{i}\right)$, where $\mathrm{D}^{-1}\left(y_{1}\right), \ldots, \mathrm{D}^{-1}\left(y_{k}\right) \neq \emptyset$ and $y_{1}>y_{2}>\ldots>y_{k}>y^{\prime}$ (resp. $y_{1}<y_{2}<\ldots<y_{k}<y^{\prime}$ ) for all $y^{\prime} \in\left[a_{y}, b_{y}\right] \backslash\{y\}$.

- RLO $k$-maximum (resp. minimum) (Record): Given a range $\mathfrak{R}=\left[a_{x}, b_{x}\right] \times$ $\left[a_{y}, b_{y}\right] \subset\left[0,2^{\ell}\right) \times\left[0,2^{\ell}\right)$

If $\mathrm{D} \cap \mathfrak{R} \neq \emptyset$ : return the set of records $\left\{\left(x_{i}, y_{i}\right)\right\}_{i=1}^{k} \in \mathrm{D}$ such that $\left(x_{1}, y_{1}\right)>_{R L O}\left(x_{2}, y_{2}\right)>_{R L O} \ldots>_{R L O}\left(x_{k}, y_{k}\right)>_{R L O}\left(x^{\prime}, y^{\prime}\right)$ (resp. $\left.\left(x_{1}, y_{1}\right)<_{R L O}\left(x_{2}, y_{2}\right)<_{R L O} \ldots<_{R L O}\left(x_{k}, y_{k}\right)<_{R L O}\left(x^{\prime}, y^{\prime}\right)\right)$ for all $\left(x^{\prime}, y^{\prime}\right) \in(\mathrm{D} \cap \mathfrak{R}) \backslash\{(x, y)\}$.

If $\mathrm{D} \cap \mathfrak{R}=\emptyset$ : return $\emptyset$. 
Moving from minimum (resp. maximum) queries their $k$ variants elements queries is straightforward, we simply modify the ranges to use $x_{k}$ and $y_{k}$ instead of $x, y$, assuming that $\left(x_{k}, y_{k}\right)$ is the $k$-th element returned. Similar to minimum and maximum queries, RLO $k$-minimum/maximum queries over records can only support ranges where $\left[a_{y}, b_{y}\right]$ is of polynomial length while $\left[a_{x}, b_{x}\right]$ can be of exponential length.

Remark 3. For the queries presented in this section, the variants over records using lexicographic order can be accomplished by using the techniques that enable those queries over keys.

\section{C.4 Queries on the Sub-Databases, $D^{-1}(y)$, of D}

Alluded to in previous queries, unlike ZK-EDB, ZK-EEDB allows a committer to prove correctness of queries pertaining to the set, $\mathrm{D}^{-1}(y)$, of all records that share the same value in D. This is due to the second Merkle tree, which contains commitments to the individual sub-databases $\mathrm{D}^{-1}(y)$ within its leaves.

In almost all cases, the queries discussed in previous sections can be specialized to $\mathrm{D}^{-1}(y)$ for any desired value $y$. Particularly, membership, range and $(k-)$ minimum/maximum over keys in $\mathrm{D}^{-1}(y)$ can be proven in zero-knowledge by applying the same query over $\mathrm{D}$ but with the "special" range $\mathfrak{R}=\left[a_{x}, b_{x}\right] \times[y, y]$. The only query which does not directly translate to $\mathrm{D}^{-1}(y)$ is the nearest neighbour query. In the way it is formulated in Section C.1, if there are no keys in $\mathrm{D}^{-1}(y)$, then some key with another value $y^{\prime} \neq y$ will be returned. This strictly is not a nearest neighbour query on $\mathrm{D}^{-1}(y)$, but the procedures to generate a proof can be easily modified to simply return $\emptyset$, along with a proof that the correctly committed digest $\operatorname{com}_{\mathrm{D}_{y}^{-1}}$ at leaf $y$ of $\operatorname{com}_{\mathrm{D}^{-1}}$ is a soft commitment, should $\mathrm{D}^{-1}(y)=\emptyset$.

\section{Formal Security Definitions of ZK-EDB}

- Completeness: For all databases D and for all keys $x$, it holds that

$$
\begin{aligned}
& \operatorname{Pr}\left[\text { crs } \leftarrow \operatorname{Init}\left(1^{\lambda}\right) ;(\operatorname{com}, \Delta) \leftarrow \operatorname{ComDB}(\text { crs }, \mathrm{D}) ;\right. \\
& \Pi_{x} \leftarrow \operatorname{ProveQ}(\text { crs }, \mathrm{D},(\text { com }, \Delta), x) ; \\
& \left.\quad \operatorname{Verify}\left(\text { crs }, \text { com }, x, \Pi_{x}\right)=\mathrm{D}(x)\right]=1-\nu(\lambda),
\end{aligned}
$$

for some negligible function $\nu(\cdot)$.

- Soundness: For all keys $x$ and for any PPT algorithm $\mathrm{P}^{\prime}$,

$$
\begin{aligned}
\operatorname{Pr}[\text { crs } & \leftarrow \operatorname{Init}\left(1^{\lambda}\right) ;\left(\text { com }, x, \Pi_{x}, \Pi_{x}^{\prime}\right) \leftarrow \mathrm{P}^{\prime}(\text { crs }) ; \\
& \left(\operatorname{Verify} \mathrm{Q}\left(\text { crs }, \text { com }, x, \Pi_{x}\right)=y \neq b a d\right) \\
\wedge & \left(\operatorname{VerifyQ}\left(\text { crs }, \text { com }, x, \Pi_{x}^{\prime}\right)=y^{\prime} \neq b a d\right) \\
\wedge & \left.\left(y \neq y^{\prime}\right)\right]
\end{aligned}
$$

is negligible. 
- Zero-Knowledge: For any PPT adversary $\mathcal{A}$ and any efficiently computable database $\mathrm{D}$, there exists an effcient simulator for EDB with three algorithms (SInit, SimCommitDB, SProveQ ${ }^{\mathrm{D}}$ ) such that the results of the following two experiments are indistinguishable:

Real experiment:

1. Let $c r s \leftarrow \operatorname{lnit}\left(1^{\lambda}\right),(\mathrm{com}, \Delta) \leftarrow \operatorname{ComDB}(\mathrm{crs}, \mathrm{D})$ and $s_{0}=\Pi_{0}=\varepsilon$.

2. For $1 \leq i \leq n$, we have $\left(x_{i}, s_{i}\right) \leftarrow \mathcal{A}\left(\mathrm{crs}, \mathrm{com}, \Pi_{0}, \ldots, \Pi_{i-1}, s_{i-1} 0\right)$ and $\mathcal{A}$ gets a real proof $\Pi_{i}=\operatorname{ProveQ}\left(\right.$ crs $, \mathrm{D},($ com,$\left.\Delta), x_{i}\right)$.

The experiment outputs $\left(\mathrm{crs}, x_{1}, \Pi_{1}, \ldots, x_{n}, \Pi_{n}\right)$.

Ideal experiment:

1. Let $\left(c r s^{\prime}, s t_{0}\right) \leftarrow \operatorname{SInit}\left(1^{\lambda}\right),\left(c o m^{\prime}, s t_{1}\right) \leftarrow \operatorname{SCom}\left(s t_{0}\right)$ and $s_{0}=\Pi_{0}^{\prime}=\varepsilon$.

2. For $1 \leq i \leq n$, we have $\left(x_{i}, s_{i}\right) \leftarrow \mathcal{A}\left(\operatorname{crs}^{\prime}, \operatorname{com}^{\prime}, \Pi_{0}^{\prime}, \ldots, \Pi_{i-1}^{\prime}, s_{i-1}\right)$ and $\mathcal{A}$ gets a simulated proof $\Pi_{i}^{\prime} \leftarrow \operatorname{SProveQ}^{\mathrm{D}}\left(c r s^{\prime}, s t_{1}, x_{i}\right)$.

The experiment outputs $\left(\operatorname{crs}^{\prime}, x_{1}, \Pi_{1}^{\prime}, \ldots, x_{n}, \Pi_{n}^{\prime}\right)$.

SProve $Q^{D}$ is an oracle that is allowed to invoke a database oracle $D$ and obtain values $\mathrm{D}(x)$ for any key $x$ chosen by the adversary $\mathcal{A}$.

\section{E A Table of Comparison between Size-Hiding Zero-Knowledge Databases.}

\begin{tabular}{|r|c|c|c|c|c|c|}
\hline & \multicolumn{2}{|c|}{ ZK-EDB } & \multicolumn{2}{|c|}{ ZK-EDB + NIZK } & \multicolumn{2}{c|}{ Our Work, ZK-EEDB } \\
\cline { 2 - 7 } & Yes & No & Yes & No & Yes & No \\
\hline \hline Mem (Key) & \multicolumn{2}{|c|}{$\mathcal{O}(\ell)$} & \multicolumn{2}{|c|}{$\mathcal{O}(\ell)$} & \multicolumn{2}{|c|}{$\mathcal{O}(\ell)$} \\
\hline Mem (Value) & \multicolumn{2}{|c|}{$\boldsymbol{x}$} & \multicolumn{2}{|c|}{$\boldsymbol{x}$} & $\mathcal{O}(|\mathcal{L}| \ell)$ & $\mathcal{O}(\ell)$ \\
\hline Mem (Record) & $\mathcal{O}(\ell)$ & $\boldsymbol{x}$ & $\mathcal{O}(\ell)$ & $|\mathrm{NIZK}|$ & \multicolumn{2}{|c|}{$\mathcal{O}(\ell)$} \\
\hline Range (Key) & $\mathcal{O}(|\mathcal{L}| \ell)$ & $\mathcal{O}(I \ell)$ & $\mathcal{O}(|\mathcal{L}| \ell)$ & $\mathcal{O}(I \ell)$ & $\mathcal{O}(|\mathcal{L}| \ell)$ & $\mathcal{O}(I \ell)$ \\
\hline Range (Value) & \multicolumn{2}{|c|}{$\boldsymbol{x}$} & \multicolumn{2}{|c|}{$\boldsymbol{x}$} & $\mathcal{O}\left(\left(K^{\prime}+|\mathcal{L}|\right) \ell\right)$ & $\mathcal{O}(I \ell)$ \\
\hline Range (Record) & \multicolumn{2}{|c|}{$\boldsymbol{x}$} & \multicolumn{2}{|c|}{$L_{x}|\mathrm{NIZK}|$} & $\mathcal{O}\left(L_{y}(1+K) \ell\right)$ & $\mathcal{O}\left(L_{y}\left(1+I_{x}\right) \ell\right)$ \\
\hline
\end{tabular}

Table 1. Comparison of Proof Sizes for ZK-EDB, ZK-EDB+NIZK and Our Work.

"Yes" denotes the case where there are records in the database that satisfy the query and "No" corresponds to the case where there are no records that satisfy the query. Range is $[a, b]$ for values and keys, $\left[a_{x}, b_{x}\right] \times\left[a_{y}, b_{y}\right]$ for records and $\mathcal{L}$ is the answer. $I=\log (b-a), I_{x}=\log \left(b_{x}-a_{x}\right), L_{x}=\left(b_{x}-a_{x}\right), L_{y}=\left(b_{y}-a_{y}\right)$ and $K^{\prime}=V(1+K)$, where $\mathrm{V}$ is the number of distinct values in $\mathcal{L}$ and $\mathrm{K}$ is the largest size amongst the sets of records that have the same value. 


\section{F A Trapdoor Mercurial Commitment from Ideal Lattices}

This section presents an adaptation of the trapdoor mercurial commitment scheme from Section 5 into the ring setting. To this end, we employ the ideallattice-based techniques and tools from [22] and [8]. Modulo some minor changes in parameters and the underlying computational assumption, the description of the resulting scheme and its security analysis are almost identical to the one in Section 5 .

\section{F.1 Background}

Let $n$ be a power of 2 and let $q \in \operatorname{poly}(n)$. We will work with $\operatorname{rings} \mathcal{R}=$ $\mathbb{Z}[X] /\left(\Phi_{2 n}(X)\right)$ and $\mathcal{R}_{q}=(\mathcal{R} / q \mathcal{R})$, where $\Phi_{2 n}(X)=X^{n}+1$ is the cyclotomic polynomial of degree $n$. For $\mathbf{A} \in \mathcal{R}_{q}^{1 \times m}$, define the $q$-ary lattice

$$
\Lambda^{\perp}(\mathbf{A})=\left\{\mathbf{x} \in \mathcal{R}^{m}: \mathbf{A} \cdot \mathbf{x}=0\right\} .
$$

Definition 2 ( RingSIS $\left._{n, m, q, \beta}\right)$. Given uniformly random $\mathbf{A} \in \mathcal{R}_{q}^{1 \times m}$, find a non-zero $\mathbf{v} \in \Lambda^{\perp}(\mathbf{A})$ such that $\|\mathbf{v}\| \leq \beta$.

The discrete Gaussian distribution over the $\operatorname{ring} D_{\mathcal{R}, \sigma} \equiv D_{\mathbb{Z}^{n}, \sigma}$ is defined by identifying the ring $\mathcal{R}$ as $\mathbb{Z}^{n}$ under the coefficient embedding. We will need the following results from [8].

Lemma 7 ([8]). Let $q$ be a power of $3, \bar{m} \geq 2\lceil\log q\rceil+3$ and $\sigma \geq \omega(\sqrt{\log n \bar{m}})$. With overwhelming probability over the choice of $\mathbf{A} \hookleftarrow U\left(\mathcal{R}_{q}^{1 \times \bar{m}}\right)$, if $\mathbf{r} \hookleftarrow D_{\mathcal{R}^{\bar{m}}, \sigma}$, we have:

1. $\mathbf{A} \cdot \mathbf{r}$ is within negligible statistical distance from the uniform distribution over $\mathcal{R}_{q}$.

2. For any non-zero $\mathbf{V} \in \mathcal{R}^{1 \times \bar{m}}$, the conditional min-entropy of $\mathbf{V} \cdot \mathbf{r}$ given $\mathbf{A} \cdot \mathbf{r}$ is at least $\Omega(n)$.

As in 8 , we will work with $q=3^{w}$, a power of 3 , and define the gadget matrix $\mathbf{G}$ as a row vector of ring elements: $\mathbf{G}=\left[1,3,9, \ldots, 3^{w-1}\right] \in \mathcal{R}^{1 \times w}$. Let $\bar{m}=2\lceil\log q\rceil+3$ and $m=\bar{m}+w$. If $\mathbf{R} \in \mathcal{R}^{\bar{m} \times w}$ is a small-norm matrix and if $\mathbf{A}=[\overline{\mathbf{A}} \mid \mathbf{G}-\overline{\mathbf{A}} \cdot \mathbf{R}] \in \mathcal{R}_{q}^{1 \times m}$, where $\overline{\mathbf{A}} \in \mathcal{R}_{q}^{1 \times \bar{m}}$, then $\mathbf{R}$ is a trapdoor for $\mathbf{A}$.

Lemma 8 ([228]). Let $n, q, w, \bar{m}, m$ be as above. Then, there exists a PPT algorithm $\operatorname{RTrapGen}(n, m, q)$ that outputs a matrix $\mathbf{A} \in \mathcal{R}_{q}^{1 \times m}$ together with a trapdoor $\mathbf{R} \in \mathcal{R}^{\bar{m} \times w}$, such that the distribution of $\mathbf{A}$ is statistically close to uniform.

Moreover, for any $\mathbf{u} \in \mathcal{R}_{q}$ and $\sigma=\Omega(\sqrt{n \log q \log n})$, there exists a PPT algorithm $\mathrm{RSampleD}(\mathbf{R}, \mathbf{A}, \mathbf{u}, \sigma)$ that outputs $\mathbf{r} \in \mathcal{R}^{m}$ sampled from a distribution statistically close to $D_{\Lambda^{\mathbf{u}}(\mathbf{A}), \sigma}$.

As shown in [22], a trapdoor for matrix $\mathbf{A} \in \mathcal{R}_{q}^{1 \times m}$ can be efficiently extended into a trapdoor for any matrix $\mathbf{B} \in \mathcal{R}_{q}^{1 \times(m+w)}$ of the form $\mathbf{B}=\left[\mathbf{A} \mid \mathbf{A}^{\prime}\right]$, where matrix $\mathbf{A}^{\prime} \in \mathcal{R}_{q}^{1 \times w}$. 


\section{F.2 Construction}

Let $\lambda \in \mathbb{N}$ be a security parameter. We denote by $\mathcal{R}_{0,1}$ the set of all elements of $\mathcal{R}$ that have coefficients in $\{0,1\}$. The scheme works with message space $\mathcal{M}=\mathcal{R}_{0,1}^{l}$, for $l \in \operatorname{poly}(\lambda)$. Let $n=\mathcal{O}(\lambda)$ be a power of 2 , and let $q=\widetilde{\mathcal{O}}\left(l \cdot n^{3}+n^{5}\right)$ be such that $q=3^{w}$. Let $\bar{m}=2\lceil\log q\rceil+3$ and $m=\bar{m}+w$. Choose a Gaussian parameter $\sigma=\Omega(\sqrt{n \log q \log n})$.

$-(m p k, m s k) \leftarrow \operatorname{Setup}\left(1^{\lambda}\right)$ : Choose a matrix $\mathbf{A}_{0} \hookleftarrow U\left(\mathcal{R}_{q}^{1 \times l}\right)$. Run algorithm $\operatorname{RTrapGen}(n, m, q)$ (Lemma 8) to generate a pair $\left(\mathbf{A}_{1}, \mathbf{T}\right)$, where $\mathbf{A}_{1} \in \mathcal{R}_{q}^{1 \times m}$ is statistically close to uniform and $\mathbf{T} \in \mathcal{R}^{\bar{m} \times w}$ is its trapdoor.

Output $m p k=\left(\mathbf{A}_{0}, \mathbf{A}_{1}\right)$ and $m s k=\mathbf{T}$.

- $\mathbf{C} \leftarrow \mathbb{H}$ Commit $(m p k, \boldsymbol{\mu} ;(\mathbf{R}, \mathbf{r}))$ : Given a message $\boldsymbol{\mu} \in \mathcal{R}_{0,1}^{l}$ and randomness $\mathbf{R} \hookleftarrow D_{\mathcal{R}^{m \times w}, \sigma}$ and $\mathbf{r} \hookleftarrow D_{\mathcal{R}^{m+w}, \sigma}$, define $\mathbf{B}=\left[\mathbf{A}_{1} \mid \mathbf{B}_{1}\right] \in \mathcal{R}_{q}^{1 \times(m+w)}$, where $\mathbf{B}_{1}=\mathbf{A}_{1} \cdot \mathbf{R} \in \mathcal{R}_{q}^{1 \times w}$. Then, compute $\mathbf{c}=\mathbf{A}_{0} \cdot \boldsymbol{\mu}+\mathbf{B} \cdot \mathbf{r} \in \mathcal{R}_{q}$ and output the hard commitment $\mathbf{C}=\left(\mathbf{c}, \mathbf{B}_{1}\right) \in \mathcal{R}_{q} \times \mathcal{R}_{q}^{1 \times w}$.

$-\pi \leftarrow \mathbb{H}$ Open $\left(m p k, \mu ;(\mathbf{R}, \mathbf{r})\right.$ : Output $\pi=(\mathbf{R}, \mathbf{r}) \in \mathcal{R}^{m \times w} \times \mathcal{R}^{m+w}$.

- $\mathbb{H}$ Verify $(m p k, \boldsymbol{\mu}, \mathbf{C}, \pi)$ : Given a commitment $\mathbf{C}=\left(\mathbf{c}, \mathbf{B}_{1}\right) \in \mathcal{R}_{q} \times \mathcal{R}_{q}^{1 \times w}$ and a purported hard opening $\pi=(\mathbf{R}, \mathbf{r})$, proceed as follows.

1. Return 0 if $\mathbf{R}=\left[\mathbf{r}_{1}|\ldots| \mathbf{r}_{w}\right]$ has a column such that $\left\|\mathbf{r}_{i}\right\|>\sigma \sqrt{n m}$ or if $\|\mathbf{r}\|>\sigma \sqrt{n(m+w)}$.

2. Let $\mathbf{B}=\left[\mathbf{A}_{1} \mid \mathbf{B}_{1}\right] \in \mathcal{R}_{q}^{1 \times(m+w)}$. Return 1 if $\mathbf{B}_{1}=\mathbf{A}_{1} \cdot \mathbf{R}$ and $\mathbf{c}=$ $\mathbf{A}_{0} \cdot \boldsymbol{\mu}+\mathbf{B} \cdot \mathbf{r}$.

$-\mathbf{C} \leftarrow \mathbb{S C o m m i t}(m p k ;(\mathbf{R}, \mathbf{r}))$ : Given $\mathbf{R} \hookleftarrow D_{\mathcal{R}^{m \times w}, \sigma}$ and $\mathbf{r} \hookleftarrow D_{\mathcal{R}^{m+w}, \sigma}$, compute the matrix $\mathbf{B}=\left[\mathbf{A}_{1} \mid \mathbf{G}-\mathbf{A}_{1} \cdot \mathbf{R}\right] \in \mathcal{R}_{q}^{1 \times(m+w)}$ and $\mathbf{c}=\mathbf{B} \cdot \mathbf{r} \in \mathcal{R}_{q}$. Output $\mathbf{C}=\left(\mathbf{c}, \mathbf{B}_{1}\right) \in \mathcal{R}_{q} \times \mathcal{R}_{q}^{1 \times w}$, where $\mathbf{B}_{1}=\mathbf{G}-\mathbf{A}_{1} \cdot \mathbf{R}$. Note that matrix $\mathbf{R}$ is a trapdoor for $\mathbf{B}$.

$-\tau \leftarrow \operatorname{SOpen}(m p k, \boldsymbol{\mu}$, flag; $(\mathbf{R}, \mathbf{r}))$ :

- If flag $=\mathbb{S}$, we must have $\mathbf{C}=\left(\mathbf{c}, \mathbf{B}_{1}\right)=\left(\mathbf{B} \cdot \mathbf{r}, \mathbf{G}-\mathbf{A}_{1} \cdot \mathbf{R}\right)$. Compute $\mathbf{c}^{\prime}=\mathbf{c}-\mathbf{A}_{0} \cdot \boldsymbol{\mu}$ and sample $\mathbf{r}^{\prime} \leftarrow \operatorname{RSampleD}\left(\mathbf{R}, \mathbf{B}, \mathbf{c}^{\prime}, \sigma\right)$ (Lemma 8).

Then, output $\tau=\mathbf{r}^{\prime} \in \mathcal{R}^{m+w}$, which satisfies $\mathbf{c}=\mathbf{A}_{0} \cdot \boldsymbol{\mu}+\mathbf{B} \cdot \mathbf{r}^{\prime}$ and $\left\|\mathbf{r}^{\prime}\right\| \leq \sigma \sqrt{n(m+w)}$ with overwhelming probability (Lemma 1 ).

- If flag $=\mathbb{H}$, output $\tau=\mathbf{r} \in \mathcal{R}^{m+w}$.

- $\operatorname{SVerify}(m p k, \boldsymbol{\mu}, \mathbf{C}, \tau)$ : Let $\mathbf{C}=\left(\mathbf{c}, \mathbf{B}_{1}\right) \in \mathcal{R}_{q} \times \mathcal{R}_{q}^{1 \times w}$ and $\tau=\mathbf{r} \in \mathcal{R}^{m+w}$ and define $\mathbf{B}=\left[\mathbf{A}_{1} \mid \mathbf{B}_{1}\right] \in \mathcal{R}_{q}^{1 \times(m+w)}$. Return 1 if $\mathbf{c}=\mathbf{A}_{0} \cdot \boldsymbol{\mu}+\mathbf{B} \cdot \mathbf{r}$ and $\|\mathbf{r}\| \leq \sigma \sqrt{n(m+w)}$. Otherwise, return 0.

- $\mathbf{C} \leftarrow \operatorname{MFake}(m p k ;(\mathbf{R}, \mathbf{r}))$ : Given $\mathbf{R} \hookleftarrow D_{\mathcal{R}^{m \times w}, \sigma}$ and $\mathbf{r} \hookleftarrow D_{\mathcal{R}^{m+w}, \sigma}$, compute $\mathbf{B}=\left[\mathbf{A}_{1} \mid \mathbf{B}_{1}\right] \in \mathcal{R}_{q}^{1 \times(m+w)}$, where $\mathbf{B}_{1}=\mathbf{A}_{1} \cdot \mathbf{R}$, and compute $\mathbf{c}=\mathbf{B} \cdot \mathbf{r}$. Output $\mathbf{C}=\left(\mathbf{c}, \mathbf{B}_{1}\right)$. 
$-\pi \leftarrow \mathbb{H}$ Equivocate $(m s k, \boldsymbol{\mu} ;(\mathbf{R}, \mathbf{r}))$ : Let $m s k=\mathbf{T}$ and let the fake commitment be $\mathbf{C}=\left(\mathbf{c}, \mathbf{B}_{1}\right)=\left(\mathbf{B} \cdot \mathbf{r}, \mathbf{A}_{1} \cdot \mathbf{R}\right)$, where $\mathbf{B}=\left[\mathbf{A}_{1} \mid \mathbf{A}_{1} \cdot \mathbf{R}\right]$. Compute $\mathbf{c}^{\prime}=\mathbf{c}-\mathbf{A}_{0} \cdot \boldsymbol{\mu}$. Then, extend $\mathbf{T}$ into a trapdoor $\mathbf{T}_{\mathbf{B}}$ for the matrix $\mathbf{B}=\left[\mathbf{A}_{1} \mid \mathbf{A}_{1} \cdot \mathbf{R}\right]$ and sample $\mathbf{r}^{\prime} \leftarrow \operatorname{RSampleD}\left(\mathbf{T}_{\mathbf{B}}, \mathbf{B}, \mathbf{c}^{\prime}, \sigma\right)$. Output $\pi=\left(\mathbf{R}, \mathbf{r}^{\prime}\right) \in \mathcal{R}^{m \times w} \times \mathcal{R}^{m+w}$.

$-\tau \leftarrow$ SEquivocate $(m s k, \boldsymbol{\mu} ;(\mathbf{R}, \mathbf{r}))$ : Let $m s k=\mathbf{T}$ and let the fake commitment be $\mathbf{C}=\left(\mathbf{c}, \mathbf{B}_{1}\right)=\left(\mathbf{B} \cdot \mathbf{r}, \mathbf{A}_{1} \cdot \mathbf{R}\right)$, where $\mathbf{B}=\left[\mathbf{A}_{1} \mid \mathbf{A}_{1} \cdot \mathbf{R}\right]$. Compute $\mathbf{c}^{\prime}=$ $\mathbf{c}-\mathbf{A}_{0} \cdot \boldsymbol{\mu}$. Then, extend $\mathbf{T}$ into a trapdoor $\mathbf{T}_{\mathbf{B}}$ for the matrix $\mathbf{B}=\left[\mathbf{A}_{1} \mid \mathbf{A}_{1} \cdot \mathbf{R}\right]$ and sample $\mathbf{r}^{\prime} \leftarrow \operatorname{RSampleD}\left(\mathbf{T}_{\mathbf{B}}, \mathbf{B}, \mathbf{c}^{\prime}, \sigma\right)$. Output $\tau=\mathbf{r}^{\prime} \in \mathcal{R}^{m+w}$.

$-\left(\mathbf{R}^{\prime}, \mathbf{r}^{\prime}\right) \leftarrow$ FakeExplain $(m s k ;(\mathbf{R}, \mathbf{r}))$ : Given $m s k=\mathbf{T}$ together with a Gaussian matrix $\mathbf{R}=\left[\mathbf{r}_{1}|\ldots| \mathbf{r}_{w}\right] \hookleftarrow D_{\mathbb{Z}^{m \times w}, \sigma}$ and a vector $\mathbf{r} \hookleftarrow D_{\mathcal{R}^{m+w}, \sigma}$ such that $\mathbf{C}=\left(\mathbf{c}, \mathbf{B}_{1}\right)=\left(\mathbf{B} \cdot \mathbf{r}, \mathbf{A}_{1} \cdot \mathbf{R}\right)$ is a fake commitment, set $\mathbf{r}^{\prime}=\mathbf{r}$ and use the trapdoor $\mathbf{T}$ for $\mathbf{A}_{1}$ to sample a small-norm $\mathbf{R}^{\prime}=\left[\mathbf{r}_{1}^{\prime}|\ldots| \mathbf{r}_{w}^{\prime}\right]$ such that $\mathbf{A}_{1} \cdot \mathbf{R}^{\prime}=\mathbf{G}-\mathbf{A}_{1} \cdot \mathbf{R}$. To do this, let $\mathbf{G}=\left[\mathbf{g}_{1}|\ldots| \mathbf{g}_{w}\right]$, and for each $i \in[w]$, sample $\mathbf{r}_{i}^{\prime} \leftarrow$ RSampleD $\left(\mathbf{T}, \mathbf{A}_{1}, \mathbf{g}_{i}-\mathbf{A}_{1} \cdot \mathbf{r}_{i}\right)$.

Then, output $\left(\mathbf{R}^{\prime}, \mathbf{r}^{\prime}\right)$ which satisfy $\mathbf{C}=\left(\mathbf{c}, \mathbf{B}_{1}\right)=\left(\mathbf{B} \cdot \mathbf{r}^{\prime}, \mathbf{G}-\mathbf{A}_{1} \cdot \mathbf{R}^{\prime}\right)$.

\section{F.3 Analysis}

We prove that the trapdoor mercurial commitment scheme described in Section F.2 satisfies the correctness and security properties defined in Section 2.1 .

Correctness. By Lemma 1, with overwhelming probability, samples from discrete Gaussian distributions $D_{\mathcal{R}^{m}, \sigma}$ and $D_{\mathcal{R}^{m+w}, \sigma}$ have their Euclidean norms bounded by $\sigma \sqrt{n m}$ and $\sigma \sqrt{n(m+w)}$, respectively. Moreover, the outputs of RSampleD are statistically close to discrete Gaussian samples, by Lemma 8. Therefore, if proofs $\pi$ and $\tau$ are generated as in Section F.2, then they should pass the verifications for Euclidean norms performed by algorithms $\mathbb{H}$ Verify and SVerify. Note further that the equations modulo $q$ verified by these algorithms must hold by construction. As a result, the scheme is correct with overwhelming probability.

Security. In the following lemmas, we show that the proposed scheme satisfies mercurial-binding under the SIS assumption, and HH, HS, SS and SE equivocation in the statistical sense.

Lemma 9. The scheme is mercurial-binding under the RingSIS ${ }_{n, m, q, \beta}$ assumption, with $\beta=\sigma n l \sqrt{n \bar{m}}+2 \sigma^{2} n \sqrt{n \bar{m}\left(m+\sigma^{2} n m^{2} w\right)(m+w)}$.

Proof. Since the scheme is a proper mercurial commitment (i.e., hard openings contain their corresponding soft opening as a proper subset), we only need to consider the hard-soft case. Towards a contradiction, let us assume that the adversary can come up with a commitment $\mathbf{C}=\left(\mathbf{c}, \mathbf{B}_{1}\right) \in \mathcal{R}_{q} \times \mathcal{R}_{q}^{1 \times w}$ which it can hard-open to a message $\boldsymbol{\mu}$ and soft-opened to a different message $\boldsymbol{\mu}^{\prime}$. This means that the adversary can output $(\boldsymbol{\mu}, \mathbf{R}, \mathbf{r}) \in \mathcal{R}_{0,1}^{l} \times \mathcal{R}^{m \times w} \times \mathcal{R}^{m+w}$ and $\left(\boldsymbol{\mu}^{\prime}, \mathbf{r}^{\prime}\right) \in \mathcal{R}_{0,1}^{l} \times \mathcal{R}^{m+w}$ such that $\mathbf{B}_{1}=\mathbf{A}_{1} \cdot \mathbf{R}$ and

$$
\mathbf{c}=\mathbf{A}_{0} \cdot \boldsymbol{\mu}+\left[\mathbf{A}_{1} \mid \mathbf{A}_{1} \cdot \mathbf{R}\right] \cdot \mathbf{r}=\mathbf{A}_{0} \cdot \boldsymbol{\mu}^{\prime}+\left[\mathbf{A}_{1} \mid \mathbf{A}_{1} \cdot \mathbf{R}\right] \cdot \mathbf{r}^{\prime} .
$$


Assuming that such a mercurial-binding adversary $\mathcal{A}$ exists, we can build a RingSIS $_{n, m, q, \beta}$ solver $\mathcal{B}$ which takes as input a RingSIS ${ }_{n, m, q, \beta}$ instance $\mathbf{A} \in \mathcal{R}_{q}^{1 \times \bar{m}}$ and finds a non-zero vector $\mathbf{v}^{\star} \in \mathcal{R}^{\bar{m}}$ of $\Lambda^{\perp}(\mathbf{A})$ such that $\left\|\mathbf{v}^{\star}\right\| \leq \beta$. To this end, $\mathcal{B}$ samples $\mathbf{R}_{0} \hookleftarrow D_{\mathcal{R}, \sigma}^{\bar{m} \times l}, \mathbf{R}_{1} \hookleftarrow D_{\mathcal{R}, \sigma}^{\bar{m} \times m}$ and defines

$$
\mathbf{A}_{0}=\mathbf{A} \cdot \mathbf{R}_{0} \in \mathcal{R}_{q}^{1 \times l}, \quad \mathbf{A}_{1}=\mathbf{A} \cdot \mathbf{R}_{1} \in \mathcal{R}_{q}^{1 \times m} .
$$

Note that, by Lemma 7, matrices $\mathbf{A}_{0}$ and $\mathbf{A}_{1}$ are statistically close to the distributions $U\left(\mathcal{R}_{q}^{1 \times l}\right)$ and $U\left(\mathcal{R}_{q}^{1 \times m}\right)$, respectively. The adversary $\mathcal{A}$ is given $m p k=\left(\mathbf{A}_{0}, \mathbf{A}_{1}\right)$ and, assuming that it can output $(\boldsymbol{\mu}, \mathbf{R}, \mathbf{r})$ and $\left(\boldsymbol{\mu}^{\prime}, \mathbf{r}^{\prime}\right)$ satisfying (5) for distinct $\boldsymbol{\mu} \neq \boldsymbol{\mu}^{\prime}$, we have

$$
\left.\mathbf{A}_{0} \cdot\left(\boldsymbol{\mu}-\boldsymbol{\mu}^{\prime}\right)\right)=\mathbf{A}_{1} \cdot\left[\mathbf{A}_{1} \mid \mathbf{A}_{1} \cdot \mathbf{R}\right] \cdot\left(\mathbf{r}^{\prime}-\mathbf{r}\right) .
$$

This implies that

$$
\mathbf{v}^{\star}=\mathbf{R}_{0} \cdot\left(\boldsymbol{\mu}-\boldsymbol{\mu}^{\prime}\right)+\left[\mathbf{R}_{1} \mid \mathbf{R}_{1} \cdot \mathbf{R}\right] \cdot\left(\mathbf{r}-\mathbf{r}^{\prime}\right) \quad \in \mathcal{R}^{\bar{m}}
$$

is a short vector of $\Lambda^{\perp}(\mathbf{A})$ with norm

$$
\left\|\mathbf{v}^{\star}\right\| \leq \sigma n l \sqrt{n \bar{m}}+2 \sigma^{2} n \sqrt{n \bar{m}\left(m+\sigma^{2} n m^{2} w\right)(m+w)} .
$$

Moreover, we claim that it is non-zero with overwhelming probability. Indeed, $\left(\boldsymbol{\mu}-\boldsymbol{\mu}^{\prime}\right) \in\{-1,0,1\}^{l}$ has at least one non-zero coordinate by hypothesis. Given that the columns of $\mathbf{R}_{0}$ have at least $\Omega(n)$ bits of min-entropy conditionally on $\mathbf{A}_{0}=\mathbf{A} \cdot \mathbf{R}_{0}$ (by Lemma 7), the product $\mathbf{R}_{0} \cdot\left(\boldsymbol{\mu}-\boldsymbol{\mu}^{\prime}\right)$ is a linear combination (with coefficients in $\{-1,0,1\}$ ) of the columns of $\mathbf{R}_{0}$ which contains a completely unpredictable term. Hence, the right-hand-side member of (6) can only cancel over $\mathcal{R}^{\bar{m}}$ with negligible probability.

Lemma 10. The scheme provides $H H, H S, S S$ and $S E$ equivocation in the statistical sense.

Proof. We show that fake commitments and their hard equivocations have a distribution which is statistically close to that of hard commitments and their hard openings.

We note that $\mathbf{B}_{1}$ is generated in the same way in both fake and hard commitments. Moreover, since $\mathbf{A}_{1}$ is statistically uniform over $\mathcal{R}_{q}^{1 \times m}$, Lemma 7 implies that the distribution $\left\{\left(\mathbf{A}_{1}, \mathbf{B}_{1}\right)=\left(\mathbf{A}_{1}, \mathbf{A}_{1} \cdot \mathbf{R}\right) \mid \mathbf{R} \hookleftarrow D_{\mathcal{R}^{m \times w}, \sigma}\right\}$ is statistically close to the distribution $U\left(\mathcal{R}_{q}^{1 \times m}\right) \times U\left(\mathcal{R}_{q}^{1 \times w}\right)$, meaning $\mathbf{B} \sim U\left(\mathcal{R}_{q}^{1 \times(m+w)}\right)$ in both hard and fake commitments. By applying Lemma 7 again, we find that the distribution of fake commitments $\left(\mathbf{c}, \mathbf{B}_{1}\right)$, given by

$$
\left\{\left(\left[\mathbf{A}_{1} \mid \mathbf{B}_{1}\right] \cdot \mathbf{r}, \mathbf{B}_{1}\right) \mid \mathbf{r} \hookleftarrow D_{\mathcal{R}^{m+w}, \sigma}\right\},
$$

is in turn statistically close to $U\left(\mathcal{R}_{q}\right) \times U\left(\mathcal{R}_{q}^{1 \times(m+w)}\right)$. This implies that the distribution of fake commitments remains statistically unchanged if we compute $\mathbf{c}$ as $\mathbf{c}=\mathbf{A} \cdot \boldsymbol{\mu}+\mathbf{B} \cdot \mathbf{r}$ instead of $\mathbf{c}=\mathbf{B} \cdot \mathbf{r}$. We call ideal ${ }_{1}$ this modification 
of the ideal experiment. Moreover, by Lemma 7 again, we know that, for any statistically uniform matrix $\mathbf{A} \sim U\left(\mathcal{R}_{q}^{1 \times(m+w)}\right)$, the distribution

$$
\left\{(\mathbf{A}, \mathbf{A} \cdot \mathbf{r}, \mathbf{r}) \in \mathcal{R}_{q}^{1 \times(m+w)} \times \mathcal{R}_{q} \times \mathcal{R}^{m+w} \mid \mathbf{r} \hookleftarrow D_{\mathcal{R}^{m+w}, \sigma}\right\}
$$

is statistically close to

$$
\left\{(\mathbf{A}, \mathbf{u}, \mathbf{r}) \in \mathcal{R}_{q}^{1 \times(m+w)} \times \mathcal{R}_{q} \times \mathcal{R}^{m+w} \mid \mathbf{u} \hookleftarrow U\left(\mathcal{R}_{q}\right), \mathbf{r} \hookleftarrow D_{\Lambda^{\mathrm{u}}(\mathbf{A}), \sigma}\right\} .
$$

Consequently, we can modify ideal ${ }_{1}$ by changing the way to equivocate the fake commitment. Instead of using extending $\mathbf{T}$ into a trapdoor for $\mathbf{B}=\left[\mathbf{A}_{1} \mid \mathbf{B}_{1}\right]$ and using it to sample $\mathbf{r}$ in a coset of the lattice $\Lambda^{\perp}(\mathbf{B})$, we just reveal the vector $\mathbf{r} \hookleftarrow D_{\mathcal{R}^{m+w}, \sigma}$ that was used to compute $\mathbf{c}=\mathbf{A} \cdot \boldsymbol{\mu}+\mathbf{B} \cdot \mathbf{r}$. If we call this experiment ideal $_{2}$, we find it statistically indistinguishable from the ideal experiment thanks to the statistical closeness of (7)-(8). We observe that ideal ${ }_{2}$ is nothing but the real $\mathrm{HH}$ equivocation experiment since $\mathbf{B}_{1}$ is generated in the same way in both experiments. This shows the HH equivocation property. The HS and SS equivocation properties can be shown in a completely similar way.

As for the SE equivocation property, it follows from two observations. First, Lemma 2 implies that the distributions

$D_{\text {fake }}:=\left\{\mathbf{A}_{1} \cdot \mathbf{R} \mid \mathbf{R} \hookleftarrow D_{\mathcal{R}^{m \times w}, \sigma}\right\}, \quad D_{\text {soft }}:=\left\{\mathbf{G}-\mathbf{A}_{1} \cdot \mathbf{R}^{\prime} \mid \mathbf{R}^{\prime} \hookleftarrow D_{\mathcal{R}^{m \times w}, \sigma}\right\}$

are both statistically close to $U\left(\mathcal{R}_{q}^{1 \times w}\right)$. Hence, the adversary's view remains statistically the same if the ideal experiment is altered to generate fake commitments where $\mathbf{B}_{1}$ is sampled from $D_{\text {soft }}$ instead of $D_{\text {fake. }}$ Moreover, since distributions (7) and (8) are statistically close, $\mathcal{A}$ 's view remains statistically the same after modification. instead of using the trapdoor $\mathbf{T}$ of $\Lambda^{\perp}\left(\mathbf{A}_{1}\right)$, we reveal the Gaussian matrix $\mathbf{R}^{\prime}$, used to get $\mathbf{B}_{1}=\mathbf{G}-\mathbf{A}_{1} \cdot \mathbf{R}^{\prime}$ after sampling $\mathbf{R}^{\prime} \hookleftarrow D_{\mathcal{R}^{m \times w}, \sigma}$. With this, the result is identical to the real game, proving the SE property. 